\title{
A generalised Green-Julg theorem for proper groupoids and Banach algebras
}

\author{
Walther Paravicini
}

\begin{abstract}
The Green-Julg theorem states that $\mathrm{K}_{0}^{G}(B) \cong \mathrm{K}_{0}\left(\mathrm{~L}^{1}(G, B)\right)$ for every compact group $G$ and every $G-C^{*}$-algebra $B$. We formulate a generalisation of this result to proper groupoids and Banach algebras. This is a key result in the proof that the Bost assembly map is surjective for proper Banach algebra coefficients.
\end{abstract}

Mathematics Subject Classification (2010). 43A20, 19K35, 22A22; 22D15.

Keywords. Green-Julg theorem, locally compact groupoid, Baum-Connes conjecture, Bost conjecture, Banach algebra.

Let $\mathcal{E}$ be a proper locally compact Hausdorff groupoid with unit space $X$ and assume that $\mathcal{E}$ carries a Haar system. In [Tu99], the following $C^{*}$-algebraic theorem is proved, which reduces to the classical Green-Julg theorem if $\mathcal{G}$ is a compact group. ${ }^{1}$

Theorem (Tu). If $\mathscr{G}$ is $\sigma$-compact and proper and if $B$ is a $\mathcal{G}-C^{*}$-algebra, then there is a canonical isomorphism

$$
\mathrm{KK}_{\mathscr{E}}\left(\bigodot_{0}(X), B\right) \cong \mathrm{KK}_{X / \mathscr{E}}\left(\bigodot_{0}(X / \mathscr{E}), B \rtimes_{r} \mathscr{\mathcal { H }}\right) \text {. }
$$

In order to translate this theorem into the setting of Banach algebras, we choose the language of V. Lafforgue's bivariant K-theory KK ${ }^{\text {ban }}$, introduced in [Laf02] and [Laf06]. More precisely, we proceed as follows:

- We replace the $\mathcal{E}$-C $\mathrm{C}^{*}$-algebra $B$ by a $\mathcal{E}$-Banach algebra, so the left-hand side of (1) should then be replaced by ${ }^{2} \mathrm{KK}_{\mathscr{E}}^{\mathrm{ban}}\left(\mathcal{C}_{0}(X), B\right)$.

- The crossed product of $B$ with $\mathscr{E}$ is replaced by $\mathcal{A}(\mathcal{E}, B)$, where $\mathcal{A}(\mathscr{E})$ is some unconditional completion of $\mathcal{C}_{c}(\mathscr{\mathscr { S }})$ as, for example, $\mathrm{L}^{1}(\mathscr{\mathscr { G }})$.

\footnotetext{
${ }^{1}$ Actually, Proposition 6.25 of [Tu99] is more general than cited here: It allows $\mathrm{C}^{*}$-algebras in the first variable that are of a more general form. We confine ourselves to "trivial" coefficients in the first variable. Note that this theorem of Tu also generalises Theorem 5.4 in [KS03].

${ }^{2}$ Actually, it should be replaced by $\mathrm{KK}_{\mathscr{g}}^{\text {ban }}\left(\mathbb{C}_{X}, B\right)$ where $\mathbb{C}_{X}$ denotes the constant field over $X$ with fibre $\mathbb{C}$. We will sometimes identify $\mathscr{C}_{0}(X)$ and $\mathbb{C}_{X}$ to obtain statements of theorems which look familiar.
} 
- For technical reasons, we do not use $\mathrm{KK}_{X / \mathscr{G}}^{\mathrm{ban}}$ on the right-hand side but a variant called $\mathcal{R} \mathrm{KK}^{\text {ban }}$ which is defined in the first section of this article.

This way, we obtain the following conjecture:

Conjecture. If $\mathscr{G}$ is proper, if $B$ is a $\mathscr{G}$-Banach algebra and if $\mathcal{A}(\mathscr{G})$ is an unconditional completion of $\mathcal{C}_{c}(\mathscr{E})$, then there is a canonical isomorphism

$$
\mathrm{KK}_{\mathscr{E}}^{\mathrm{ban}}\left(\bigodot_{0}(X), B\right) \cong \mathcal{R} \mathrm{KK}^{\mathrm{ban}}\left(\bigodot_{0}(X / \mathscr{E}) ; \bigodot_{0}(X / \mathscr{E}), \mathcal{A}(\mathscr{E}, B)\right) .
$$

Note that the right-hand side reduces to $\mathrm{K}_{0}(\mathcal{A}(\mathscr{E}, B))$ if $X / \mathscr{E}$ is compact, see Corollary 1.9 .

We show this conjecture under some regularity conditions:

- Firstly, $B$ should be a non-degenerate $\mathscr{E}$-Banach algebra, i.e., the span of $B B$ is dense in $B$. It is conceivable that this condition can be removed but the price one has to pay is the technical challenge of adapting the definition of $\mathrm{KK}^{\mathrm{ban}}$ and its variants to make it suitable also for degenerate Banach algebras.

- Secondly, we want $\mathcal{E}$ to carry a cut-off function (which is automatic if $X / \mathscr{E}$ is $\sigma$ compact). The first two conditions allow us to define a canonical homomorphism $J_{\mathcal{A}}^{B}$ from the left-hand side to the right-hand side of (2).

- Thirdly, to show that this homomorphism $J_{\mathscr{A}}^{B}$ is bijective, we need to impose some conditions on the unconditional completion $\mathcal{A}(\mathscr{E})$ :

- If we let the unconditional completion $\mathcal{A}(\mathscr{E})$ be regular (this notion will be explained in Section 4.3; the completion $\mathrm{L}^{1}(\mathscr{E})$ and its symmetrised version $\mathrm{L}^{1}(\mathscr{E}) \cap \mathrm{L}^{1}(\mathscr{E})^{*}$ are regular), then we are able to define a canonical homomorphism from the right-hand side to the left-hand side of (2) and show that $J_{\mathcal{A}}^{B}$ is split surjective.

- For split injectivity, we need that $\mathscr{A}(\mathscr{E})$ satisfies some additional regularity condition (which is true if $\mathcal{A}(\mathscr{E})$ equals $\mathrm{L}^{1}(\mathscr{E})$ or its symmetrised version). The proof of the injectivity part is only sketched in this article, and the reader is referred to [Par07] for the details.

- It is conceivable that one can show that the right-hand side is independent of $\mathcal{A}(\mathscr{E})$, which would settle the conjecture without any conditions on $\mathcal{A}(\mathscr{G})$. We have partial results in this direction, for example, in the case that $\mathscr{G}=G \ltimes X$, where $G$ is a locally compact group acting properly and co-compactly on a locally compact space $X$, see Corollary 2.5 and Proposition A.2.

Note that, already from the surjectivity part of the generalised Green-Julg Theorem, it is possible to deduce the split surjectivity of the Bost assembly map for proper Banach algebra coefficients as carried out in [Par13b].

In the first section of this article, we introduce a variant $\mathcal{R} \mathrm{KK}^{\text {ban }}$ of $\mathrm{KK}^{\text {ban }}$ for Banach algebras which carry an action of $\mathcal{C}_{0}(X)$, where $X$ is a locally compact 
Hausdorff space. This theory serves as a recipient for the descent homomorphism and also appears on the right-hand side of the generalised Green-Julg theorem. It has the feature that if $X$ is compact, then

$$
\mathcal{R K K}_{G}^{\mathrm{ban}}(\mathcal{C}(X) ; A \otimes \mathcal{C}(X), B) \cong \mathrm{KK}_{G}^{\mathrm{ban}}(A, B) \text {. }
$$

The second section introduces the main theorem in more detail, outlines the proof and gives some corollaries.

The third section makes some useful tools and concepts available for the proof of the main theorem, e.g., cut-off pairs (a refinement of cut-off functions) and monotone completions (a refinement of unconditional completions).

The fourth section contains the actual proof of the generalised Green-Julg theorem.

In an appendix, the dependence of $\mathrm{K}_{*}(\mathcal{A}(\mathcal{E}, B))$, for proper $\mathcal{E}$, on the unconditional completion $\mathcal{A}(\mathscr{E})$ is studied.

Most of the results of this article are contained in the doctoral thesis [Par07], which comprises full proofs and all technical details; I would like to thank my Ph.D. supervisor Siegfried Echterhoff. I also thank Vincent Lafforgue, who has drawn my attention to the study of the Bost conjecture for proper Banach algebra coefficients, for his helpful advice. This research has been supported by the Deutsche Forschungsgemeinschaft (SFB 478).

Notation. All Banach spaces and Banach algebras that appear in this article are supposed to be complex. References which explain the necessary notation and the concepts to understand Banach algebras that carry actions of groupoids are [Laf06] and [Par09a].

\section{1. $\mathcal{C}_{0}(X)$-Banach algebras and $\mathscr{R} \mathrm{KK}^{\mathrm{ban}}$-theory}

Let $X$ be a locally compact Hausdorff space. The notion of a $\ell_{0}(X)$ - $\mathrm{C}^{*}$-algebra is well-known in the literature, and it has already been generalised to the concept of a $\ell_{0}(X)$-Banach algebra, see [Blan96]. For $\bigodot_{0}(X)-C^{*}$-algebras, there is a natural variant of KK-theory called $\mathcal{R K K}$. This section is dedicated to the development of an analogous theory for $\ell_{0}(X)$-Banach algebras. This can be thought of as an intermediate step between $\mathrm{KK}^{\text {ban }}$ for ordinary Banach algebras as defined in [Laf02] and the variant of $\mathrm{KK}^{\text {ban }}$ for fields of Banach algebras as defined in [Laf06].

The starting point for our definition of $\mathcal{R K K}$ is the following observation: If $A$ and $B$ are $\bigodot_{0}(X)-C^{*}$-algebras and $(E, T)$ is a cycle for $\mathcal{R K K}\left(\mathcal{C}_{0}(X) ; A, B\right)$, then $E$ carries a canonical action of $\mathcal{C}_{0}(X)$ defined through the identification $E \cong E \otimes_{B} B$, just let $\varphi_{0}(X)$ act on the second factor. This action is the unique action of $\ell_{0}(X)$ on $E$ that is compatible with the module action of $B$. The usual condition on an $\mathcal{R K K}$-cycle, namely that $(\chi a)(e b)=(a e)(\chi b)$ for all $a \in A, e \in E, b \in B$ and $\chi \in \mathcal{C}_{0}(X)$, then just means that the actions of $\mathcal{C}_{0}(X)$ on $A$ and $E$ should be 
compatible. So $E$ is what could be called a $\ell_{0}(X)$-Hilbert $A$ - $B$-module. The corner stone for the definition of $\mathcal{R} \mathrm{KK}^{\mathrm{ban}}$ should hence be the notion of a $\mathcal{C}_{0}(X)$-Banach $A$ $B$-pair (if $A$ and $B$ are $\mathcal{C}_{0}(X)$-Banach algebras). The fundamental notion underlying all this is a notion of a $\mathcal{C}_{0}(X)$-Banach space, which turns out to be rather simple:

1.1. $\mathscr{C}_{0}(X)$-Banach spaces, $\boldsymbol{C}_{0}(X)$-Banach algebras, etc. A $\mathscr{C}_{0}(X)$-Banach space $E$ is by definition a non-degenerate Banach $\mathcal{C}_{0}(X)$-module, where non-degeneracy means that $\mathcal{C}_{0}(X) \cdot E$ is (dense in) $E$. If $E$ and $F$ are $\mathcal{C}_{0}(X)$-Banach spaces, then we take the bounded linear $\ell_{0}(X)$-linear maps from $E$ to $F$ as morphisms from $E$ to $F$. We are going to denote the morphisms from $E$ to $F$ by $\mathrm{L}^{\ell_{0}(X)}(E, F)$.

If $E$ is a Banach space, then $E X=\mathscr{C}_{0}(X, E)$ is a $\mathscr{C}_{0}(X)$-Banach space with the canonical action of $\mathcal{C}_{0}(X)$.

Let $E_{1}$ and $E_{2}$ be $\ell_{0}(X)$-Banach spaces. Let $E_{1} \times E_{2}$ be the product Banach space (with the sup-norm). Then $E_{1} \times E_{2}$ is a $\ell_{0}(X)$-Banach space with the obvious product action. Similarly, there is a notion of the sum $E_{1} \oplus E_{2}$ of $\mathcal{C}_{0}(X)$-Banach spaces $E_{1}$ and $E_{2}$ using the sum-norm. It is compatible with the $\mathcal{C}_{0}(X)$-tensor product that we are going to define below. Let $F$ be another $\ell_{0}(X)$-Banach space. A $\mathbb{C}$-bilinear map $\mu: E_{1} \times E_{2} \rightarrow F$ is called $\mathcal{C}_{0}(X)$-bilinear if $\mu$ is $\ell_{0}(X)$-linear in every component. There is a universal space $E_{1} \otimes \mathcal{e}_{0}(X) E_{2}$ for continuous $\mathscr{C}_{0}(X)$ bilinear maps on $E_{1} \times E_{2}$, called the $\mathcal{C}_{0}(X)$-tensor product. It can be constructed as a quotient of the projective tensor product $E_{1} \otimes^{\pi} E_{2}$ and is itself a $\mathcal{C}_{0}(X)$-Banach space in an obvious way.

Definition 1.1. A $\ell_{0}(X)$-Banach algebra $B$ is a Banach algebra $B$ which is at the same time a $\ell_{0}(X)$-Banach space such that the multiplication of $B$ is $\mathcal{C}_{0}(X)$-bilinear.

A homomorphism of $\mathcal{C}_{0}(X)$-Banach algebras $\varphi: A \rightarrow B$ is simply a contractive $\ell_{0}(X)$-linear homomorphism $\varphi$ of algebras.

For the rest of Section 1.1, let $A, B$ and $C$ be $\ell_{0}(X)$-Banach algebras.

We define the fibrewise unitalisation of $B$ to be $B \oplus \mathcal{C}_{0}(X)$. The norm on $B \oplus$ $\mathcal{C}_{0}(X)$ is the sum-norm and multiplication is given by $(b, \varphi) \cdot(c, \psi):=(b c+\psi b+$ $\varphi c, \varphi \psi)$ for all $b, c \in B, \varphi, \psi \in \mathcal{C}_{0}(X)$. In the theory of $\mathcal{C}_{0}(X)$-Banach algebras, the fibrewise unitalisation is the adequate substitute for the ordinary unitalisation, e.g., it should be used in the definition of pushouts along homomorphisms of $\mathcal{C}_{0}(X)$-Banach algebras. We will not stress this technical point in what follows.

A $C_{0}(X)$-Banach $B$-module is a Banach $B$-module $E$ which is at the same time a $\mathscr{C}_{0}(X)$-Banach space such that the module action is $\ell_{0}(X)$-bilinear. We define $\mathcal{C}_{0}(X)$-Banach $B$ - $C$-bimodules analogously. Let $E, F$ be $\mathcal{C}_{0}(X)$-Banach $B$ modules. Then we write $\mathrm{L}_{B}^{\mathcal{C}_{0}(X)}(E, F)$ for the subspace of $\mathrm{L}_{B}(E, F)$ of operators which are also $\mathcal{C}_{0}(X)$-linear. Note that if $E$ is a non-degenerate Banach $B$-module, then all elements of $\mathrm{L}_{B}(E, F)$ are automatically $C_{0}(X)$-linear.

There is also an obvious notion of homomorphisms with coefficient maps between $\ell_{0}(X)$-Banach modules, compare the definition in [Par09b]. 
Let $E$ be a right $\ell_{0}(X)$-Banach $B$-module and let $F$ be a left $\ell_{0}(X)$-Banach $B$-module. The balanced $\mathcal{C}_{0}(X)$-tensor product $E \otimes_{B}^{\ell_{0}(X)} F$ of $E$ and $F$ over $B$ is defined to be the universal object for the $B$-balanced $\mathcal{C}_{0}(X)$-multilinear maps on $E \times F$. It can be obtained by taking $E \otimes_{B} F$ and dividing out elements of the form $e \varphi \otimes f-e \otimes \varphi f$. Note that if $E$ or $F$ is $B$-non-degenerate, then it is not hard to show that the usual balanced tensor product and the balanced $\mathcal{C}_{0}(X)$-tensor product agree: $E \otimes_{B}^{\ell_{0}(X)} F=E \otimes_{B} F$.

The pushout along homomorphisms of $\ell_{0}(X)$-Banach algebras is defined as in the ordinary case, compare [Laf02], p. 12, but using the fibrewise unitalisation defined above. It has the expected (functorial) properties.

Definition 1.2. Let $B$ be a $\digamma_{0}(X)$-Banach algebra. A $\bigodot_{0}(X)$-Banach $B$-pair $E$ is a $B$-pair $E$ such that $E^{<}$and $E^{>}$are $\ell_{0}(X)$-Banach $B$-modules and such that the inner product is $\mathcal{C}_{0}(X)$-bilinear. If $A$ is another $\mathcal{C}_{0}(X)$-Banach algebra, then a Banach $A$-B-pair $E$ is a $\mathscr{C}_{0}(X)$-Banach $A$-B-pair if it is a $\mathcal{C}_{0}(X)$-Banach $B$-pair and the actions of $A$ on $E^{<}$and $E^{>}$are $\mathcal{C}_{0}(X)$-bilinear.

For example, if $B$ is a $\mathcal{C}_{0}(X)$-Banach algebra, then $(B, B)$ is a $\mathscr{C}_{0}(X)$-Banach $B$-pair.

Let $E$ and $F$ be $\ell_{0}(X)$-Banach $B$-pairs. Then an element $T$ of $\mathrm{L}_{B}(E, F)$ is called $\ell_{0}(X)$-linear if $T^{<}$and $T^{>}$are $\ell_{0}(X)$-linear. The subspace of all $\ell_{0}(X)$-linear maps in $\mathrm{L}_{B}(E, F)$ is denoted by $\mathrm{L}_{B}^{C_{0}(X)}(E, F)$.

The definitions of concurrent homomorphisms with coefficient maps between $\ell_{0}(X)$-Banach pairs, the $\ell_{0}(X)$-tensor product of $\ell_{0}(X)$-Banach pairs and the pushout of $\ell_{0}(X)$-Banach pairs along homomorphisms of $\ell_{0}(X)$-Banach algebras are the obvious variation of the corresponding definitions for ordinary Banach pairs, requiring all maps to be $\ell_{0}(X)$-linear (compare the discussion for Banach modules above).

Proposition 1.3. Let $E$ and $F$ be $\mathcal{C}_{0}(X)$-Banach B-pairs. Then $\mathrm{K}_{B}(E, F)$ is always contained in $\mathrm{L}_{B}^{\mathcal{C}_{0}(X)}(E, F)$, i.e., $\mathcal{C}_{0}(X)$-linearity is automatic for compact operators.

Proof. Let $f^{>} \in F^{>}$and $e^{<} \in E^{<}$. Let $T:=\left|f^{>}\right\rangle\left\langle e^{<}\right|$. To show that $T^{>}$is $\mathcal{C}_{0}(X)$-linear let $e^{>} \in E^{>}$and $\varphi \in \mathscr{C}_{0}(X)$. Then

$$
T^{>}\left(\varphi e^{>}\right)=f^{>}\left\langle e^{<}, \varphi e^{>}\right\rangle=f^{>}\left(\varphi\left\langle e^{<}, e^{>}\right\rangle\right)=\varphi\left(f^{>}\left\langle e^{<}, e^{>}\right\rangle\right)=\varphi T^{>}\left(e^{>}\right) .
$$

Similarly one shows that $T^{<}$is $\ell_{0}(X)$-linear. Now the set of all $\ell_{0}(X)$-linear elements in $\mathrm{L}_{B}(E, F)$ is a closed subspace, so it contains the whole of $\mathrm{K}_{B}(E, F)$.

It is easy to see that $\mathrm{K}_{B}(E, F)$ is a $\ell_{0}(X)$-Banach space and that the canonical bilinear map from $F^{>} \times E^{<}$to $\mathrm{K}_{B}(E, F)$ is $\mathcal{C}_{0}(X)$-bilinear. If $G$ is another $\ell_{0}(X)$ Banach $B$-pair, then the composition of elements of $\mathrm{K}_{B}(F, G)$ and $\mathrm{K}_{B}(E, F)$ is $\bigodot_{0}(X)$-bilinear. In particular, $\mathrm{K}_{B}(E)$ is a $\ell_{0}(X)$-Banach algebra. 
Definition 1.4. Let $E$ and $F$ be $\mathcal{C}_{0}(X)$-Banach $B$-pairs. Then $T \in \mathrm{L}_{B}(E, F)$ is called locally compact if $\chi T$ is compact for all $\chi \in \mathscr{C}_{0}(X)$.

Note that it suffices to check $\chi T \in \mathrm{K}_{B}(E, F)$ for all $\chi \in \ell_{c}(X)$. Note also that locally compact operators are automatically $\ell_{0}(X)$-linear. The bounded locally compact operators form a closed subset of $\mathrm{L}_{B}^{\ell_{0}(X)}(E, F)$.

\section{2. $\mathcal{R} K_{G}^{\mathrm{ban}}\left(\mathcal{C}_{0}(X) ; A, B\right)$}

1.2.1. Gradings and group actions. A graded $\ell_{0}(X)$-Banach space is a $\ell_{0}(X)$ Banach space $E$ endowed with a grading automorphism commuting with the $\ell_{0}(X)$ action.

Let $G$ be a locally compact Hausdorff group that acts continuously on $X$. Note that $\mathcal{C}_{0}(X)$ is a $G$-Banach algebra when equipped with the $G$-action $(g \chi)(x):=\chi\left(g^{-1} x\right)$, $\chi \in \mathscr{C}_{0}(X), g \in G, x \in X$. A $G-\mathscr{C}_{0}(X)$-Banach space is a $G$-Banach space $E$ which is at the same time a $\mathscr{C}_{0}(X)$-Banach space such that the actions of $G$ and $\mathscr{C}_{0}(X)$ are compatible in the following sense:

$$
g(\chi e)=(g \chi)(g e), \quad \chi \in \mathcal{C}_{0}(X), g \in G, e \in E,
$$

i.e., the product $\ell_{0}(X) \times E \rightarrow E$ is $G$-equivariant.

From these definitions we also get an obvious definition of a graded $G-\ell_{0}(X)$ Banach space. Taking this as a starting point one can define graded $G-\bigodot_{0}(X)$-Banach algebras and graded equivariant homomorphisms between them, graded $G-\mathcal{C}_{0}(X)$ Banach pairs, etc.

\subsubsection{Definition of $\mathcal{R} \mathrm{KK}_{G}^{\mathrm{ban}}\left(\mathcal{C}_{0}(X) ; A, B\right)$}

Definition 1.5. Let $A$ and $B$ be $G-\bigodot_{0}(X)$-Banach algebras. Then the class $\mathbb{E}_{G}^{\text {ban }}\left(\mathcal{C}_{0}(X) ; A, B\right)$ is defined to be the class of pairs $(E, T)$ such that $E$ is a nondegenerate graded $G-\ell_{0}(X)$-Banach $A-B$-pair and, if we forget the $\ell_{0}(X)$-structure, the pair $(E, T)$ is an element of $\mathbb{E}_{G}^{\mathrm{ban}}(A, B)$. Note that $T$ in the definition is automatically $\mathcal{C}_{0}(X)$-linear because $E$ is non-degenerate.

The constructions one usually performs with $\mathrm{KK}^{\text {ban }}$-cycles are obviously compatible with the additional $\ell_{0}(X)$-structure, so we can form the sum of $\mathrm{KK}^{\mathrm{ban}}$-cycles and take their pushout along homomorphisms of $G-\ell_{0}(X)$-Banach algebras. We also have a $\ell_{0}(X)$-linear notion of morphisms of $\mathrm{KK}^{\text {ban }}$-cycles, giving us a $\ell_{0}(X)$-linear version of isomorphisms of $\mathrm{KK}^{\text {ban }}$-cycles. Hence also the notion of homotopy makes sense in the $\mathcal{C}_{0}(X)$-setting so we can formulate the following definition:

Definition 1.6. The class of all homotopy classes of elements of $\mathbb{E}_{G}^{\mathrm{ban}}\left(\mathcal{C}_{0}(X) ; A, B\right)$ is denoted by $\mathcal{R} \mathrm{KK}_{G}^{\mathrm{ban}}\left(\mathcal{C}_{0}(X) ; A, B\right)$. The sum of cycles induces a law of composition on $\mathcal{R K K}_{G}^{\text {ban }}\left(\mathcal{C}_{0}(X) ; A, B\right)$ making it an abelian group. 
The fact that the composition on $\mathcal{R K K}_{G}^{\mathrm{ban}}\left(\mathcal{C}_{0}(X) ; A, B\right)$ has inverses can be proved just as in the case without the $\ell_{0}(X)$-structure, i.e., Lemme 1.2.5 of [Laf02] and its proof are compatible with the additional $\ell_{0}(X)$-module action. There is an obvious forgetful group homomorphism

$$
\mathcal{R K K}_{G}^{\mathrm{ban}}\left(\mathcal{C}_{0}(X) ; A, B\right) \rightarrow \mathrm{KK}_{G}^{\mathrm{ban}}(A, B) .
$$

1.3. Comparison with the $\mathbf{K K}^{\mathrm{ban}}$-theory for fields of Banach algebras. Let $\boldsymbol{G}$ be a locally compact Hausdorff groupoid with unit space $X$. In [Laf06], V. Lafforgue has introduced an equivariant $\mathrm{KK}^{\mathrm{ban}}$-theory for $\mathcal{E}$-Banach algebras. A $\mathcal{E}$-Banach algebra is, in particular, an upper semi-continuous field of Banach algebras over $X$. If $A$ is such a field, then one can consider $\Gamma_{0}(X, A)$, the space of all sections of $A$ which vanish at infinity. The Banach algebra $\Gamma_{0}(X, A)$ carries a canonical action of $\digamma_{0}(X)$ making it a $\bigodot_{0}(X)$-Banach algebra. However, it is not clear how to find an elegant way to model a general $\mathscr{E}$-action on $A$ on the level of elements of $\Gamma_{0}(X, A)$. Nevertheless, it is rather straightforward in the case that $\mathcal{E}=G \ltimes X$ where $G$ is a locally compact Hausdorff group acting on $X$. In this case, $\Gamma_{0}(X, A)$ is a $G-\ell_{0}(X)$ Banach algebra in a canonical fashion. We have the following result whose proof can be found in [Par07], Section 4.7.

Proposition 1.7. Let $A$ and $B$ be $G \ltimes X$-Banach algebras. Then there is a canonical isomorphism

$$
\mathrm{KK}_{G \ltimes X}^{\mathrm{ban}}(A, B) \cong \mathcal{R} \mathrm{KK}_{G}^{\mathrm{ban}}\left(\bigodot_{0}(X) ; \Gamma_{0}(X, A), \Gamma_{0}(X, B)\right) .
$$

Conversely, start with a $G-\bigodot_{0}(X)$-Banach algebra $\mathcal{A}$. For all $x \in X$, the quotient Banach algebra $\mathcal{A}_{x}=\mathscr{A} /\left(\mathcal{C}_{0}(X \backslash\{x\}) \mathcal{A}\right)$ is called the fibre of $\mathcal{A}$ over $x$; it comes with a natural quotient map $\mathcal{A} \ni a \mapsto a_{x} \in \mathcal{A}_{x}$. One can regard $\mathfrak{F}(\mathcal{A}):=\left(\mathcal{A}_{x}\right)_{x \in X}$ as a $G \ltimes X$-Banach algebra. Let us denote the $G-\bigodot_{0}(X)$-Banach algebra $\Gamma_{0}(X, \mathfrak{F}(\mathcal{A}))$ by $(\mathcal{S}(\mathcal{A})$ and call it the Gelfand transform of $\mathcal{A}$. There is a canonical homomorphism $\iota_{\mathcal{A}}$ from $\mathcal{A}$ to $\mathscr{S}(\mathcal{A})$ which sends every $a \in \mathcal{A}$ to the section $x \mapsto a_{x} \in \mathcal{A}_{x}$. Sadly enough, $\iota_{\mathcal{A}}$ needs neither be injective nor surjective, we only know that it has dense image; we do not have $\mathcal{A} \cong \Gamma_{0}(X, \mathfrak{F}(\mathcal{A}))$ in general. The homomorphism $\iota_{\mathcal{A}}$ is isometric (and therefore an isomorphism) if and only if the $\mathcal{C}_{0}(X)$-Banach algebra $\mathcal{A}$ is what is called locally $\ell_{0}(X)$-convex, i.e., for all $\chi_{1}, \chi_{2} \in \ell_{0}(X), \chi_{1}, \chi_{2} \geq 0$, $\chi_{1}+\chi_{2} \leq 1$ and all $a_{1}, a_{2} \in A$ we have

$$
\left\|\chi_{1} a_{1}+\chi_{2} a_{2}\right\| \leq \max \left\{\left\|a_{1}\right\|,\left\|a_{2}\right\|\right\}
$$

see [Gie82] and also Appendix A.2 of [Par07].

If $A$ is a $G \ltimes X$-Banach algebra, then $\Gamma_{0}(X, A)$ is automatically locally $\bigodot_{0}(X)$ convex. Actually, $A \mapsto \Gamma_{0}(X, A)$ defines an equivalence of categories between the category of $G \ltimes X$-Banach algebras and the category of locally $\bigodot_{0}(X)$-convex $G$ $\digamma_{0}(X)$-Banach algebras, the inverse functor being $\mathfrak{F}(\cdot)$. The functor $\mathcal{A} \mapsto \mathcal{S}(\mathcal{A})$ on 
the category of $G-\bigodot_{0}(X)$-Banach algebras therefore has its values in the subcategory of locally $\ell_{0}(X)$-convex $G-\complement_{0}(X)$-Banach algebras. It is a projector in the sense that $\mathbb{G}(\mathscr{G}(\mathcal{A}))$ is naturally isomorphic to $\mathscr{G}(\mathcal{A})$.

The functors $\mathfrak{F}(\cdot)$ and $\mathfrak{G}(\cdot)$ can also be applied to $G-\mathcal{C}_{0}(X)$-Banach spaces, $G-\mathcal{C}_{0}(X)$-Banach pairs etc. It is an interesting fact that $\mathfrak{F}(\cdot)$ is multiplicative in the sense that it intertwines the (fibrewise) tensor product of $G \ltimes X$-Banach spaces and the $\mathcal{C}_{0}(X)$-tensor product of $G-\mathcal{C}_{0}(X)$-Banach spaces; this can be proved using the result that the $\mathcal{C}_{0}(X)$-tensor product of locally $\mathcal{C}_{0}(X)$-convex spaces is again locally $\mathcal{C}_{0}(X)$-convex, see [Par08].

If $\mathcal{A}$ and $\mathscr{B}$ are arbitrary $G-\mathcal{C}_{0}(X)$-Banach algebras, then it is possible to construct a group homomorphism

$$
\mathcal{R K K}_{G}^{\mathrm{ban}}\left(\mathcal{C}_{0}(X) ; \mathcal{A}, \mathscr{B}\right) \rightarrow \mathcal{R} \mathrm{KK}_{G}^{\mathrm{ban}}\left(\mathcal{C}_{0}(X) ; \mathscr{G}(\mathcal{A}), \mathscr{G}(\mathscr{B})\right) .
$$

It is not clear under which conditions this is an isomorphism if $\mathcal{A}$ and $\mathscr{B}$ are not locally $\ell_{0}(X)$-convex. A first result along these lines is proved in [Par13a] showing that $\mathcal{A}$ and $\mathscr{S}(\mathcal{A})$ have the same (non-equivariant) K-theory.

To conclude, one can say that $\mathrm{KK}_{\mathscr{g}}^{\mathrm{ban}}$ and $\mathcal{R} \mathrm{KK}^{\mathrm{ban}}$ agree on the (equivalent) categories of $G \ltimes X$-Banach algebras / locally $\ell_{0}(X)$-convex $G$ - $\bigodot_{0}(X)$-Banach algebras, but on the one hand, $\mathrm{KK}_{g}^{\mathrm{ban}}$ can be extended much further to Banach algebras which carry actions of arbitrary groupoids, on the other hand, $\mathcal{R} \mathrm{KK}^{\mathrm{ban}}$ can be extended to $G-\mathcal{C}_{0}(X)$-Banach algebras which fail to be locally $\mathcal{C}_{0}(X)$-convex (and that such algebras appear naturally is the raison d'être for this theory).

A much more elaborate discussion of the two concepts can be found in Chapter 4 of [Par07].

1.4. Special case: $X$ compact. We conclude this section by discussing how $\mathcal{R} \mathrm{KK}_{G}^{\text {ban }}$ reduces to ordinary $\mathrm{KK}_{G}^{\mathrm{ban}}$-theory if $X$ is a compact space. To this end, let $G$ be a locally compact Hausdorff group and $X$ be a locally compact Hausdorff space on which $G$ acts.

Proposition 1.8. Let $A$ be a non-degenerate $G$-Banach algebra and let $B$ be a nondegenerate $G-\bigodot_{0}(X)$-Banach algebra. If $X$ is compact, then there is a canonical isomorphism

$$
\mathcal{R K K}_{G}^{\mathrm{ban}}(\mathcal{C}(X) ; A \otimes \mathcal{C}(X), B) \cong \mathrm{KK}_{G}^{\mathrm{ban}}(A, B) .
$$

If we take $A$ to be $\mathbb{C}$ with the trivial $G$-action, then $A \otimes \mathscr{C}(X)$ is isomorphic to $e(X)$ :

Corollary 1.9. Let $B$ be a non-degenerate $G-\bigodot_{0}(X)$-Banach algebra. If $X$ is compact, then

$$
\mathcal{R K K}_{G}^{\mathrm{ban}}(\mathcal{C}(X) ; \mathcal{C}(X), B) \cong \mathrm{KK}_{G}^{\mathrm{ban}}(\mathbb{C}, B) .
$$


This, together with Théorème 1.2.8 of [Laf02], implies that if $X$ is compact and $G$ is the trivial group, then

$$
\mathcal{R} \mathrm{KK}^{\mathrm{ban}}(\mathcal{C}(X) ; \mathcal{C}(X), B) \cong \mathrm{KK}^{\mathrm{ban}}(\mathbb{C}, B) \cong \mathrm{K}_{0}(B) .
$$

Proof of the Proposition 1.8. First note that the projective tensor product $A \otimes C(X)$ is a non-degenerate $G$ - $(X)$-Banach algebra so that $\mathcal{R} K_{G}^{\text {ban }}(\mathcal{C}(X) ; A \otimes \mathscr{C}(X), B)$ makes sense.

Now observe that there is a canonical forgetful homomorphism

$$
\mathcal{R} \mathrm{KK}_{G}^{\mathrm{ban}}(\mathcal{C}(X) ; A \otimes \digamma(X), B) \rightarrow \mathrm{KK}_{G}^{\mathrm{ban}}(A \otimes \ell(X), B) .
$$

Moreover, there is a canonical homomorphism $j_{A}$ of $G$-Banach algebras from $A$ to $A \otimes \mathcal{C}(X)$, namely the map $a \mapsto a \otimes 1$. This gives a group homomorphism from $\mathrm{KK}_{G}^{\mathrm{ban}}(A \otimes \ell(X) ; B)$ to $\mathrm{KK}_{G}^{\mathrm{ban}}(A, B)$. Let

$$
\kappa: \mathcal{R K K}_{G}^{\mathrm{ban}}(\mathcal{C}(X) ; A \otimes \mathcal{C}(X), B) \rightarrow \mathrm{KK}_{G}^{\mathrm{ban}}(A, B)
$$

be the composition of these two homomorphisms. We show that $\kappa$ is an isomorphism.

We first prove surjectivity: Let $(E, T) \in \mathbb{E}_{G}^{b a n}(A, B)$. Instead of defining a $\ell(X)$ structure on $E$, which we do not know how to do, we define a structure on the cycle $\left(E \otimes_{B} B, T \otimes 1\right) \in \mathbb{E}_{G}^{\mathrm{ban}}(A, B)$, where $E \otimes_{B} B=\left(B \otimes_{B} E^{<}, E^{>} \otimes_{B} B\right)$. Note that $\left(E \otimes_{B} B, T \otimes 1\right)=(E, T) \otimes_{B} \operatorname{Mor}_{G}^{\text {ban }}\left(\operatorname{Id}_{B}\right)$, so it is homotopic to $(E, T)$, see [Par09b], Proposition 5.28 (5). On $E^{>} \otimes_{B} B$ we define a canonical $C(X)$-structure: if $e^{>} \in E^{>}$and $b \in B$ and $\varphi \in \mathcal{C}(X)$, then $\varphi\left(e^{>} \otimes b\right):=e^{>} \otimes(\varphi b)$. This makes $E^{>} \otimes_{B} B$ a right $G-C(X)$-Banach $B$-module. We proceed similarly on the left-hand side. It is easy to see that $E \otimes_{B} B$ is a $G-C(X)$-Banach $B$-pair with this $\digamma(X)$-action. The operator $T \otimes 1$ is clearly $\ell(X)$-linear (which is automatic anyway, because $E \otimes_{B} B$ is non-degenerate).

Now we have to define an action of $A \otimes \mathcal{C}(X)$ on $E \otimes_{B} B:$ If $a \in A, \chi \in \mathcal{C}(X)$, $e^{>} \in E^{>}$and $b \in B$, then we define $(a \otimes \chi)\left(e^{>} \otimes b\right):=\left(a e^{>}\right) \otimes(\chi b)$. This gives an action of $A \otimes \digamma(X)$ on $E^{>} \otimes_{B} B$ making it a $G$ - $C(X)$-Banach $A \otimes \digamma(X)-B$ bimodule. A similar definition can be made for the left-hand side. We check that $A \otimes C(X)$ acts on $E \otimes_{B} B$ by elements of $\mathrm{L}_{B}\left(E \otimes_{B} B\right)$. Let therefore be $a \in A$, $\chi \in \mathcal{C}(X), e^{<} \in E^{<}, e^{>} \in E^{>}$and $b^{<}, b^{>} \in B$. Then

$$
\begin{aligned}
\left\langle b^{<} \otimes e^{<},(a \otimes \chi)\left(e^{>} \otimes b^{>}\right)\right\rangle & =\left\langle b^{<} \otimes e^{<},\left(a e^{>}\right) \otimes\left(\chi b^{>}\right)\right\rangle \\
& =b^{<}\left\langle e^{<}, a e^{>}\right\rangle\left(\chi b^{>}\right) \\
& =\left(\chi b^{<}\right)\left\langle e^{<} a, e^{>}\right\rangle b^{>} \\
& =\left\langle\left(b^{<} \otimes e^{<}\right)(a \otimes \chi), e^{>} \otimes b^{>}\right\rangle .
\end{aligned}
$$

By trilinearity and continuity of both sides, this equation can be extended from the elementary tensors to all of $A \otimes \ell(X), B \otimes_{B} E^{<}$and $E^{>} \otimes_{B} B$. So $E \otimes_{B} B$ is in $\mathbb{E}_{G}^{\mathrm{ban}}(\mathcal{C}(X) ; A \otimes \mathcal{C}(X), B)$. Applying $\kappa$ to it means forgetting the $\mathcal{C}(X)$-structure 
and reducing the $A \otimes \mathcal{C}(X)$-action back to the $A$-action on $E \otimes_{B} B$, so we are back to where we started. Hence $\kappa$ is surjective.

The same argument shows that $\kappa$ is injective: Let $\left(E_{0}, T_{0}\right)$ and $\left(E_{1}, T_{1}\right)$ be elements of the class $\mathbb{E}_{G}^{\mathrm{ban}}(\mathcal{C}(X) ; A \otimes \mathcal{C}(X), B)$ such that $\kappa\left(E_{0}, T_{0}\right)$ and $\kappa\left(E_{1}, T_{1}\right)$ are homotopic in $\mathbb{E}_{G}^{b a n}(A, B)$. Find $(E, T) \in \mathbb{E}_{G}^{b a n}(\mathcal{C}(X) ; A \otimes \mathcal{C}(X), B[0,1])$ such that $\kappa(E, T) \in \mathbb{E}_{G}^{\text {ban }}(A, B[0,1])$ is a homotopy from $\kappa\left(E_{0}, T_{0}\right)$ to $\kappa\left(E_{1}, T_{1}\right)$. Now $\mathrm{ev}_{i, *}^{B}(E, T)$ is contained in $\mathbb{E}_{G}^{\mathrm{ban}}(\mathcal{C}(X) ; A \otimes \mathcal{C}(X), B)$ for all $i \in\{0,1\}$, and $\kappa\left(\mathrm{ev}_{i, *}^{B}(E, T)\right)$ is isomorphic (in $\left.\mathbb{E}_{G}^{b a n}(A, B)\right)$ to $\left(E_{i}, T_{i}\right)$. Now $E_{i}$ is a non-degenerate $B$-pair, so it is easy to see that the $\mathscr{C}(X)$-structure on $E$ is unique. Hence the isomorphism between $\kappa\left(\mathrm{ev}_{i, *}^{B}(E, T)\right)$ and $\left(E_{i}, T_{i}\right)$ must be $\ell(X)$-linear. Also the action of $A \otimes \digamma(X)$ is uniquely determined by the actions of $A$ and $\digamma(X)$, so the isomorphism between $\kappa\left(\mathrm{ev}_{i, *}^{B}(E, T)\right)$ and $\left(E_{i}, T_{i}\right)$ must also respect this structure. In other words, it is an isomorphism of cycles in $\mathbb{E}_{G}^{b a n}(\mathcal{C}(X) ; A \otimes \ell(X), B)$. So $\left(E_{0}, T_{0}\right)$ and $\left(E_{1}, T_{1}\right)$ are homotopic. Hence $\kappa$ is injective.

\section{A generalised Green-Julg theorem}

2.1. Proper groupoids and cut-off functions. In this paragraph we recall two notions which we need for the formulation of the theorem.

Definition 2.1. A locally compact Hausdorff groupoid is called proper if the following map is proper, i.e., inverses of compact sets are compact:

$$
\mathcal{E} \rightarrow \mathscr{E}^{(0)} \times \mathcal{E}^{(0)}, \quad \gamma \mapsto(r(\gamma), s(\gamma)) .
$$

We collect some examples:

(1) Let $G$ be a locally compact Hausdorff group acting from the left on a locally compact Hausdorff space $X$. Then the transformation groupoid $G \ltimes X$ is proper if and only if the action of $G$ on $X$ is proper.

(2) More generally, if $\mathcal{E}$ is a locally compact Hausdorff groupoid and $X$ is a left $\mathcal{E}$-space, then $\mathcal{E} \ltimes X$ is proper if and only if $X$ is a proper $\mathcal{E}$-space.

(3) A locally compact Hausdorff group is proper (as a groupoid) if and only if it is compact.

Definition 2.2 ([Tu99], Définition 6.7.). A continuous function $c: X \rightarrow[0, \infty[$ is called cut-off function for $\mathcal{E}$ if

(1) $\int_{\mathscr{E} x} c(s(\gamma)) \mathrm{d} \lambda^{x}(\gamma)=1$ for all $x \in X$;

(2) $r: \operatorname{supp}(c \circ s) \rightarrow X$ is proper.

The latter condition means that $\operatorname{supp} c \cap \mathscr{E} K$ is compact for all compact subsets $K$ of $X$.

Recall from [Tu04] that there is a cut-off function for $\mathcal{E}$ if $X / \mathcal{E}$ is $\sigma$-compact. 
2.2. The theorem and some corollaries. For the remainder of Section 2 , let $\mathcal{E}$ be a locally compact proper Hausdorff groupoid with unit space $X$ and that carries $a$ Haar system $\lambda$. Let $\mathcal{A}(\mathcal{E})$ be an unconditional completion of $\mathcal{C}_{c}(\mathcal{E})$ and let $B$ be a non-degenerate $\mathcal{E}$-Banach algebra. In particular, this implies that $X / \mathcal{E}$ is a locally compact Hausdorff space.

The generalised Green-Julg theorem that we prove in this article asserts that we have an isomorphism

$$
\mathrm{KK}_{\mathscr{E}}^{\mathrm{ban}}\left(\mathcal{C}_{0}(X), B\right) \cong \mathcal{R} \mathrm{KK}^{\mathrm{ban}}\left(\mathcal{C}_{0}(X / \mathcal{E}) ; \mathcal{C}_{0}(X / \mathcal{E}), \mathcal{A}(\mathcal{E}, B)\right)
$$

if $\mathcal{G}$ is a proper groupoid. We construct this isomorphism only under certain conditions, more precisely, we proceed as follows:

(1) We define a natural homomorphism $J_{\mathscr{A}}^{B}$ from the left-hand side to the right-hand side of (3) in case that $\mathcal{G}$ admits a cut-off function.

(2) We define a natural homomorphism $M_{\mathcal{A}}^{B}$ in the other direction in case that $\mathcal{A}(\mathcal{G})$ is what we call regular, see Section 4.3.

(3) We show $J_{\mathcal{A}}^{B} \circ M_{\mathcal{A}}^{B}=$ Id if both conditions are satisfied.

(4) We sketch how to show $M_{\mathcal{A}}^{B} \circ J_{\mathcal{A}}^{B}=\operatorname{Id}$ if $\mathcal{A}(\mathcal{G})$ satisfies some additional regularity condition.

Observe that already the split surjectivity of $J_{\mathcal{A}}^{B}$ is an interesting result as it implies the split surjectivity of the Bost assembly map with proper Banach algebra coefficients, see [Par13b]. We hence state the surjectivity part of the generalised Green-Julg theorem separately:

Theorem 2.3. Let $\mathcal{A}(\mathcal{E})$ be a regular unconditional completion of $\mathcal{C}_{c}(\mathcal{E})$. Assume that there is a cut-off function for $\mathcal{E}$. Then the natural homomorphism

$$
J_{\mathcal{A}}^{B}: \operatorname{KK}_{\mathscr{E}}^{\mathrm{ban}}\left(\mathcal{C}_{0}(X), B\right) \rightarrow \mathcal{R} \mathrm{KK}^{\mathrm{ban}}\left(\mathcal{C}_{0}(X / \mathcal{E}) ; \mathcal{C}_{0}(X / \mathcal{E}), \mathcal{A}(\mathcal{E}, B)\right)
$$

is split surjective (with natural split $M_{\mathcal{A}}^{B}$ ) for all non-degenerate Banach algebras $B$.

The definition of a regular unconditional completion will be given in Section 4.3. As we have mentioned above, the injectivity part of our generalised Green-Julg theorem needs some extra regularity conditions. We now formulate the final result for further reference and we will introduce the necessary vocabulary only later (see Sections 3.4, 4.2, and 4.6).

Theorem 2.4 (Generalised Green-Julg Theorem). Let $\mathcal{E}$ be proper and let $\mathcal{A}(\mathcal{G})$ be an unconditional completion of $\mathcal{C}_{c}(\mathcal{E})$ such that there exists a pair $\mathscr{H}(\mathcal{E})$ of monotone completions of $\mathcal{C}_{c}(\mathscr{E})$ satisfying $(\mathrm{H} 1)-(\mathrm{H} 4)$ and such that there exists an $\mathscr{H}(\mathcal{E})$-cut-off pair for $\mathcal{G}$. Then there is an isomorphism

$$
J_{\mathcal{A}}^{B}: \operatorname{KK}_{\mathscr{G}}^{\mathrm{ban}}\left(\bigodot_{0}(X), B\right) \cong \mathcal{R} \mathrm{KK}^{\mathrm{ban}}\left(\bigodot_{0}(X / \mathcal{G}) ; \digamma_{0}(X / \mathcal{G}), \mathcal{A}(\mathcal{E}, B)\right),
$$

natural in the non-degenerate $\mathcal{E}$-Banach algebra $B$. 
The hypotheses of the theorem imply that $\mathcal{E}$ admits a cut-off function and that $\mathcal{A}(\mathscr{E})$ is regular. Hence the surjectivity part of the theorem is contained in Theorem 2.3. The proof of the injectivity part is rather lengthy and will only be sketched in Section 4.6.

Note that, according to Corollary 1.9 , the right-hand side reduces to $\mathrm{K}_{0}(\mathcal{A}(\mathcal{E}, B))$ if $X / \mathscr{E}$ is compact. In Appendix A we show that $\mathrm{K}_{0}(\mathcal{A}(\mathscr{E}, B))$ does not depend on $\mathcal{A}(\mathscr{E})$ as long as $\mathcal{A}(\mathscr{G})$ is regular or what we call strictly positive, see Definition A.3. This allows us to formulate the following corollary where the assumptions on $\mathcal{A}(\mathscr{E})$ are less restrictive than in the above theorem.

Corollary 2.5. Let $\mathcal{A}(\mathscr{G})$ be an unconditional completion of $\mathcal{C}_{c}(\mathscr{G})$ which is either regular or strictly positive. Let $X / \mathscr{G}$ be compact. Then we have natural isomorphisms

$$
\mathrm{KK}_{\mathscr{g}}^{\mathrm{ban}}\left(\mathcal{C}_{0}(X), B\right) \cong \mathcal{R} \mathrm{KK}^{\mathrm{ban}}\left(\mathcal{C}_{0}(X / \mathscr{E}) ; \mathcal{C}_{0}(X / \mathcal{E}), \mathcal{A}(\mathscr{E}, B)\right) \cong \mathrm{K}_{0}(\mathcal{A}(\mathscr{E}, B))
$$

for all non-degenerate $\mathcal{E}$-Banach algebras $B$.

Proof. As mentioned above, the second isomorphism is given by Corollary 1.9, and it is natural in $B$.

Now consider the case that $\mathcal{A}(\mathscr{E})=\mathrm{L}^{1}(\mathscr{E})$. We will see in Section 4.6 that $\mathrm{L}^{1}(\mathscr{G})$ satisfies all technical conditions that are needed for the generalised GreenJulg theorem to hold (in particular, it is regular). This shows also the first isomorphism in the special case $\mathcal{A}(\mathscr{E})=\mathrm{L}^{1}(\mathscr{E})$.

It follows from Proposition A.2 that $\mathrm{K}_{0}(\mathcal{A}(\mathcal{G}, B))$ does not depend on the particular regular or strictly positive unconditional completion $\mathcal{A}(\mathscr{E})$ in the sense that $\mathrm{K}_{0}(\mathcal{A}(\mathcal{E}, B))$ and $\mathrm{K}_{0}\left(\mathcal{A}^{\prime}(\mathcal{E}, B)\right)$ are naturally isomorphic for any pair of (regular or strictly positive) unconditional completions $\mathcal{A}(\mathscr{E})$ and $\mathcal{A}^{\prime}(\mathscr{E})$. Therefore $\mathrm{KK}_{\mathscr{G}}^{\mathrm{ban}}\left(\mathcal{C}_{0}(X), B\right)$ and $\mathrm{K}_{0}(\mathcal{A}(\mathscr{E}, B))$ are naturally isomorphic for any regular or strictly positive unconditional completion $\mathcal{A}(\mathscr{G})$. This shows the corollary.

Now consider the special case that $\mathscr{E}=G \ltimes X$ where $G$ is a locally compact Hausdorff group acting properly on a locally compact Hausdorff space $X$. Let $\mathcal{A}(G)$ be an unconditional completion of $\mathcal{C}_{c}(G)$. It is shown in Lemma A.4 that $\mathcal{A}\left(G, \mathcal{C}_{0}(X)\right)$ is a strictly positive unconditional completion of $\mathcal{C}_{c}(G \ltimes X)$, so we obtain the following corollary to Corollary 2.5 .

Corollary 2.6. Let $G$ be a locally compact Hausdorff group acting properly on a locally compact Hausdorff space $X$ such that $X / G$ is compact. If $B$ is a locally $\mathcal{C}_{0}(X)$-convex $G-\mathcal{C}_{0}(X)$-Banach algebra, then

$$
\begin{aligned}
\mathcal{R K K}_{G}^{\mathrm{ban}}\left(\mathcal{C}_{0}(X) ; \mathcal{C}_{0}(X), B\right) & \cong \mathcal{R} \mathrm{KK}^{\mathrm{ban}}\left(\mathcal{C}_{0}(X / G) ; \bigodot_{0}(X / G), \mathcal{A}(G, B)\right) \\
& \cong \mathrm{K}_{0}(\mathcal{A}(G, B))
\end{aligned}
$$

for every unconditional completion $\mathcal{A}(G)$ of $\mathcal{C}_{c}(G)$. 
The proofs of the corollaries depend heavily on the degree to which $\mathrm{K}_{0}(\mathcal{A}(\mathscr{G}, B))$ does not depend on the unconditional completion $\mathcal{A}(\mathscr{G})$. The general version of the theorem for non-compact $X / \mathcal{E}$ would be valid for arbitrary unconditional completions if one could show similar independence results for $\mathcal{R} \mathrm{KK}^{\text {ban }}$. So one is led to ask:

Question 2.7. Is $\mathcal{R} \mathrm{KK}^{\mathrm{ban}}\left(\ell_{0}(X / \mathscr{E}) ; \ell_{0}(X / \mathscr{E}), \mathcal{A}(\mathscr{E}, B)\right)$ independent of $\mathcal{A}(\mathscr{E})$ for non-compact $X / \mathscr{G}$ and arbitrary (possibly non-regular) $\mathcal{A}(\mathscr{E})$ ?

\section{A tool kit for the proof}

In this section, let $\mathscr{E}$ be a locally compact proper Hausdorff groupoid with unit space $X$ and carrying a Haar system $\lambda$. Recall that this implies that $X / \mathscr{E}$ is locally compact and Hausdorff.

3.1. Proper groupoids and the descent. Let $\mathcal{A}(\mathscr{G})$ be an unconditional completion of $\mathcal{C}_{c}(\mathscr{E})$ and let $E$ be a $\mathcal{E}$-Banach space. ${ }^{3}$ For all $\xi \in \Gamma_{c}\left(\mathcal{E}, r^{*} E\right)$ and $\chi \in \mathcal{C}_{0}(X / \mathscr{E})$ define

$$
(\chi \xi)(\gamma):=\chi(\pi(\gamma)) \xi(\gamma)
$$

for all $\gamma \in \mathscr{E}$, where $\pi$ denotes the (open) projection map $\pi: \mathscr{G} \rightarrow X / \mathcal{E}$. This defines a module action of $\mathcal{C}_{0}(X / \mathscr{E})$ on $\Gamma_{c}\left(\mathscr{E}, r^{*} E\right)$ which lifts to a module action on $\mathcal{A}(\mathscr{E}, E)$. More precisely, $\mathcal{A}(\mathscr{E}, E)$ is a non-degenerate Banach $\mathcal{C}_{0}(X / \mathscr{E})$-module, i.e., it is a $\mathcal{C}_{0}(X / \mathscr{E})$-Banach space. Note that, depending on the choice of $\mathcal{A}(\mathscr{G})$, the $\mathcal{C}_{0}(X / \mathscr{E})$-Banach space $\mathcal{A}(\mathscr{E}, E)$ does not have to be locally $\mathcal{C}_{0}(X / \mathscr{E})$-convex; it is however in important cases, e.g., if $\mathcal{A}(\mathscr{\mathscr { G }})=\mathrm{L}^{1}(\mathscr{\mathscr { G }})$.

The convolution product and also the descent of continuous linear maps respects the $\mathcal{C}_{0}(X / \mathscr{E})$-structure; in particular, if $B$ is a $\mathscr{Y}$-Banach algebra, then $\mathcal{A}(\mathscr{Y}, B)$ is not only a Banach algebra but a $\ell_{0}(X / \mathscr{E})$-Banach algebra, and if $E$ is a $\mathscr{E}$-Banach $B$-pair, then $\mathcal{A}(\mathscr{E}, E)$ is a $\mathcal{C}_{0}(X / \mathcal{E})$-Banach $\mathcal{A}(\mathscr{G}, B)$-pair, etc. Let $A$ and $B$ be $\mathcal{E}$-Banach algebras. It is not hard to show that the descent homomorphism from $\mathrm{KK}_{\mathscr{g}}^{\mathrm{ban}}(A, B)$ to $\mathrm{KK}^{\mathrm{ban}}(\mathcal{A}(\mathscr{G}, A), \mathcal{A}(\mathscr{E}, B))$ introduced in Section 1.3 of [Laf06] is indeed a homomorphism

$$
j_{\mathcal{A}}: \operatorname{KK}_{\mathscr{G}}^{\mathrm{ban}}(A, B) \rightarrow \mathcal{R} \mathrm{KK}^{\mathrm{ban}}\left(\mathcal{C}_{0}(X / \mathscr{E}) ; \mathcal{A}(\mathcal{E}, A), \mathcal{A}(\mathscr{E}, B)\right) .
$$

Because $\mathcal{A}(\mathscr{E}, B)$ does not have to be locally $\mathscr{C}_{0}(X / \mathscr{E})$-convex in general, it seems advisable to use $\mathcal{R} \mathrm{KK}^{\mathrm{ban}}$ instead of $\mathrm{KK}_{X / \mathscr{\varrho}}^{\mathrm{ban}}$, the version of $\mathrm{KK}^{\mathrm{ban}}$ for fields over $X / \mathscr{E}$.

\footnotetext{
${ }^{3}$ See [Laf06] or [Par09a] for the definitions of these concepts.
} 
3.2. Cut-off functions and cut-off pairs. Given a cut-off function $c$ as introduced in Definition 2.2, one often uses the function $c^{1 / 2}$ in the theory of $\mathrm{C}^{*}$-algebras. In the Banach algebra setting, the exponent $\frac{1}{2}$ is no longer the inevitable choice, also $c^{1 / p}$ with $1<p<\infty$ can appear quite naturally. Because we are dealing with Banach pairs rather than Banach modules, it even makes sense to extend the notion of a cut-off function as follows:

Definition 3.1. A cut-off pair for $\mathscr{E}$ is a pair $\left(c^{<}, c^{>}\right)$such that

(1) $c^{<} \in \mathcal{C}(X)_{\geq 0}$ with $r: \operatorname{supp}\left(c^{<} \circ s\right) \rightarrow X$ proper;

(2) $c^{>} \in \mathscr{C}(X)_{\geq 0}$ with $r: \operatorname{supp}\left(c^{>} \circ s\right) \rightarrow X$ proper;

(3) $\int_{\mathscr{g} x} c^{<}(s(\gamma)) c^{>}(s(\gamma)) \mathrm{d} \lambda^{x}(\gamma)=1$ for all $x \in X$.

In particular, $x \mapsto c^{<}(x) c^{>}(x)$ is a cut-off function. Conversely, if $c$ is a cut-off function for $\mathcal{G}$ and $\left.p, p^{\prime} \in\right] 1, \infty\left[\right.$ such that $\frac{1}{p}+\frac{1}{p^{\prime}}=1$, then $\left(c^{1 / p^{\prime}}, c^{1 / p}\right)$ is a cut-off pair. We can even cover the case $p=1$ :

Proposition 3.2. If $\mathcal{E}$ is such that $X / \mathcal{E}$ is $\sigma$-compact and $c$ is a cut-off function for $\mathcal{G}$, then there exists a function $d \in \mathscr{C}(X)$ with $\|d\|_{\infty}=1$ such that $(d, c)$ is a cut-off pair.

Proof. Let $\left(K_{n}\right)_{n \in \mathbb{N}}$ be an exhausting sequence of compacts in $X / \mathcal{E}$ such that $K_{n}$ is contained in the interior of $K_{n+1}$ for all $n \in \mathbb{N}$. Define $L_{n}:=\operatorname{supp} c \cap \pi^{-1}\left(K_{n}\right)$ for all $n \in \mathbb{N}$ (where $\pi$ denotes the canonical surjection from $X$ to $X / \mathscr{E}$ ). Then the $L_{n}$ are all compact. Recursively, find functions $f_{1}, f_{2}, f_{3} \ldots$ such that $f_{n} \in \mathcal{C}_{c}\left(\pi^{-1}\left(K_{n}\right)\right)$, $0 \leq f_{n} \leq 1$ and $\left.f_{n}\right|_{L_{n}} \equiv 1$ and $f_{n} \subseteq f_{n+1}$ for all $n \in \mathbb{N}$. Define $f:=\bigcup_{n \in \mathbb{N}} f_{n}$. Then this is a well-defined continuous function on $X$ such that $0 \leq f \leq 1$. It satisfies $\left.f\right|_{\text {supp } c} \equiv 1$. Moreover, it satisfies the support condition: Let $K \subseteq X / \mathscr{E}$ be compact. Find an $n \in \mathbb{N}$ such that $K \subseteq K_{n}$. Then the closed set $\pi^{-1}(K)$ is contained in $\pi^{-1}\left(K_{n}\right)$, so $\pi^{-1}(K) \cap \operatorname{supp} f$ is contained in $\pi^{-1}\left(K_{n}\right) \cap \operatorname{supp} f=$ $\pi^{-1}\left(K_{n}\right) \cap \operatorname{supp} f_{n}=\operatorname{supp} f_{n}$. Now supp $f_{n}$ is a compact subset of $\pi^{-1}\left(K_{n}\right)$, so $\pi^{-1}(K) \cap \operatorname{supp} f$ is compact as a closed subset of a compact subset.

On the level of functions with compact support, we can define a homomorphism from $\mathcal{C}_{c}(X / \mathscr{G})$ to $\mathcal{C}_{c}(\mathscr{G})$ quite generally; it is a delicate question for which completions of $\mathcal{C}_{c}(\mathscr{E})$ this homomorphism can be extended continuously to $\mathcal{C}_{0}(X / \mathscr{E})$.

Definition and Proposition 3.3. Let $\left(c^{<}, c^{>}\right)$be a cut-off pair for $\mathscr{G}$. For all $\chi \in$ $\varphi_{c}(X / \mathscr{E})$, define

$$
(\varphi(\chi))(\gamma):=c^{>}(r(\gamma)) \chi(\pi(\gamma)) c^{<}(s(\gamma))
$$

for all $\gamma \in \mathscr{E}$. Then $\varphi(\chi) \in \mathscr{C}_{c}(\mathscr{E})$, and $\varphi$ is a continuous homomorphism of algebras from $\mathscr{C}_{c}(X / \mathscr{G})$ to $\mathscr{C}_{c}(\mathscr{G})$ (with the convolution product). 
Proof. Let $\pi: X \rightarrow X / \mathcal{E}$ denote the quotient map and let $K \subseteq X / \mathcal{E}$ be the support of $\chi$. Then $K_{1}:=\operatorname{supp} c^{<} \cap \pi^{-1}(K)$ is compact in $X$ and so is $K_{2}:=\operatorname{supp} c^{>} \cap$ $\pi^{-1}(K)$. So $\left\{\gamma \in \mathcal{E}: s(\gamma) \in K_{1}, r(\gamma) \in K_{2}\right\}$ is compact and contains the support of $\varphi(\chi)$. So $\varphi(\chi) \in \mathcal{C}_{c}(\mathscr{G})$.

Let $\chi_{1}, \chi_{2} \in \mathcal{C}_{c}(\mathscr{E})$. Then for all $\gamma \in \mathscr{E}$ :

$$
\begin{aligned}
& \left(\varphi\left(\chi_{1}\right) * \varphi\left(\chi_{2}\right)\right)(\gamma) \\
& =\int_{\operatorname{gr}(\gamma)} c^{>}\left(r\left(\gamma^{\prime}\right)\right) \chi_{1}\left(\pi\left(\gamma^{\prime}\right)\right) c^{<}\left(s\left(\gamma^{\prime}\right)\right) c^{>}\left(r\left(\gamma^{\prime-1} \gamma\right)\right) \chi_{2}\left(\pi\left(\gamma^{\prime-1} \gamma\right)\right) \\
& c^{<}\left(s\left(\gamma^{\prime-1} \gamma\right)\right) \mathrm{d} \lambda^{r(\gamma)}\left(\gamma^{\prime}\right) \\
& =c^{>}(r(\gamma))\left(\chi_{1} \chi_{2}\right)(\pi(\gamma)) c^{>}(s(\gamma)) \underbrace{\int_{\operatorname{gr}(\gamma)} c^{<}\left(s\left(\gamma^{\prime}\right)\right) c^{>}\left(s\left(\gamma^{\prime}\right)\right) \mathrm{d} \lambda^{r(\gamma)}\left(\gamma^{\prime}\right)}_{=1} \\
& =\left(\varphi\left(\chi_{1} \chi_{2}\right)\right)(\gamma) .
\end{aligned}
$$

In the $\mathrm{C}^{*}$-algebra case, the interesting cut-off pair is of course $\left(c^{\frac{1}{2}}, c^{\frac{1}{2}}\right)$, where $c$ is a cut-off function for $\mathcal{E}$. In this case, ${ }^{4}$ the homomorphism $\varphi: \mathscr{\ell}_{c}(X / \mathcal{E}) \rightarrow \boldsymbol{\ell}_{c}(\mathcal{E})$ preserves the involution and can be extended to a $*$-homomorphism from $\mathcal{C}_{0}(X / \mathcal{E})$ to $\mathrm{C}_{r}^{*}(\mathscr{G})$. The pullback along this $*$-homomorphism gives a homomorphism of groups from $\mathrm{KK}_{X / \mathcal{E}}\left(\mathrm{C}_{r}^{*}(\mathscr{E}), B \rtimes_{r} \mathscr{\mathcal { H }}\right)$ to $\mathrm{KK}_{X / \mathscr{E}}\left(\mathcal{C}_{0}(X / \mathcal{E}), B \rtimes_{r} \mathscr{\mathcal { E }}\right)$.

Can the same homomorphism $\varphi: \ell_{c}(X / \mathscr{E}) \rightarrow \mathcal{C}_{c}(\mathscr{E})$ be extended to a homomorphism from $\mathcal{C}_{0}(X / \mathcal{G})$ to $\mathcal{A}(\mathscr{E})$ if $\mathcal{A}(\mathscr{E})$ is an unconditional completion of $\mathcal{C}_{c}(\mathscr{E})$ ? This would come in handy in the construction of a homomorphism from $\mathrm{KK}_{\mathscr{G}}^{\mathrm{ban}}\left(\mathcal{C}_{0}(X), B\right)$ to $\mathcal{R} \mathrm{KK}^{\text {ban }}\left(\mathcal{C}_{0}(X / \mathcal{G}) ; \boldsymbol{C}_{0}(X / \mathcal{E}), \mathcal{A}(\mathcal{H}, B)\right)$ where $B$ is a $\mathcal{H}$-Banach algebra, compare Section 2.2. One could simply take the descent homomorphism and compose it with the pullback along $\varphi$.

Apparently, $\varphi$ is not bounded even for rather elementary unconditional completions like $\mathrm{L}^{1}(\mathcal{G})$ and rather simple cut-off pairs. The construction works for $\mathrm{C}^{*}$ algebras because the choice of the cut-off pair is compatible with the norm on $\mathrm{C}_{r}^{*}(\mathscr{\mathscr { G }})$ which is defined through the action of $\mathcal{C}_{c}(\mathscr{G})$ on $\mathrm{L}^{2}(\mathscr{G})$. In Section 4.1, we will find another way to define the homomorphism for our generalised Green-Julg theorem because we do not want to deal with the technical problems that come with unbounded homomorphisms or with the compression of a Banach algebra by an unbounded projection.

Instead, we will define the homomorphism in a single step: If $E$ is a $\mathscr{E}$-Banach $B$-pair, then we are not even trying to define $\varphi^{*} \mathcal{A}(\mathcal{E}, E)$ but we consider a "smaller" space $\mathscr{D}(X, E)$, which is a (monotone) completion of $\Gamma_{c}(X, E)$. The underlying observation is that we can realise $\ell_{c}(X)$ as a subspace of $\ell_{c}(\mathscr{G})$ by the use of a cut-off pair, and this construction is compatible with the left action of $\mathcal{C}_{c}(X / \mathscr{E})$. See Section 4.1.4 for more details of this embedding.

\footnotetext{
${ }^{4}$ See Proposition 6.23 in [Tu99] for a proof.
} 
3.3. Automatic equivariance. There is a feature of proper groupoids which will prove very convenient in the upcoming sections:

Proposition 3.4. Let $A$ and $B$ be $\mathcal{E}$-Banach algebras (with $\mathcal{E}$ being proper and allowing a cut-off function). Then the operators and homotopies in the definition of $\mathrm{KK}_{g}^{\mathrm{ban}}(A, B)$ can be assumed to be $\mathscr{G}$-equivariant.

Proof. The basic idea here, as in the proof of the corresponding result for $\mathrm{C}^{*}$-algebras, is to use the cut-off function and the integration with respect to the Haar system to make given operators equivariant, compare the discussion before Proposition 6.24 in [Tu99]. On a technical level, we do this by integrating fields of operators with compact support; note that we define this integration pointwise:

Let $E$ and $F$ be $\mathcal{G}$-Banach $B$-pairs. Let $T=\left(T^{<}, T^{>}\right) \in \mathrm{L}_{r^{*} B}\left(r^{*} E, r^{*} F\right)$ have compact support. Then

$$
\int_{\mathscr{E} x} T_{\gamma} \mathrm{d} \lambda^{x}(\gamma):=\left(\int_{\mathscr{E} x} T_{\gamma}^{<} \mathrm{d} \lambda^{x}(\gamma), \int_{\mathscr{G} x} T_{\gamma}^{>} \mathrm{d} \lambda^{x}(\gamma)\right)
$$

is a continuous field of linear operators from $E$ to $F$. The same definition makes sense if $T$ has proper support, i.e., if the support of $(\chi \circ r) \cdot T$ is compact for all $\chi \in \mathcal{C}_{c}(X)$. The operator $\int_{\mathscr{G} x} T_{\gamma} \mathrm{d} \lambda^{x}(\gamma)$ is compact if $T \in \mathrm{K}_{r^{*} B}\left(r^{*} E, r^{*} F\right)$ has compact support.

We can use this procedure to produce equivariant operators. Fix a cut-off function $c$ for $\mathscr{G}$. For all $T \in \mathrm{L}_{B}(E, F)$, we define

$$
T_{x}^{\mathscr{E}}=\int_{\mathscr{E}^{x}} c(s(\gamma)) \gamma T_{s(\gamma)} \mathrm{d} \lambda^{x}(\gamma), \quad x \in X,
$$

Then $T^{\mathscr{E}}$ is an equivariant element of $\mathrm{L}_{B}(E, F)$. The construction commutes with the pushout: If $B^{\prime}$ is another $\mathcal{E}$-Banach algebra and $\varphi: B \rightarrow B^{\prime}$ is a $\mathscr{E}$-equivariant homomorphism, then $\varphi_{*}\left(T^{\mathscr{E}}\right)=\left(\varphi_{*}(T)\right)^{\mathscr{E}}$ as elements of $\mathrm{L}_{B^{\prime}}^{\mathscr{E}}\left(\varphi_{*}(E), \varphi_{*}(F)\right)$.

Now let $(E, T) \in \mathbb{E}_{\mathscr{g}}^{\mathrm{ban}}(A, B)$. Then $\left(E, T^{\mathscr{E}}\right)$ is in $\mathbb{E}_{\mathscr{g}}^{\mathrm{ban}}(A, B)$ and homotopic to $(E, T)$. To see this, let $a \in \Gamma_{c}(X, A)$. For all $x \in X$, we have

$$
a_{x}\left(T_{x}-T_{x}^{\mathscr{E}}\right)=\int_{\mathscr{g} x} c(s(\gamma)) a_{r(\gamma)}\left(T_{r(\gamma)}-\gamma T_{s(\gamma)}\right) \mathrm{d} \lambda^{x}(\gamma) .
$$

The family $\gamma \mapsto c(s(\gamma)) a_{r(\gamma)}\left(T_{r(\gamma)}-\gamma T_{s(\gamma)}\right)$ is compact and of compact support, so the integral is compact. So $T$ and $T^{\mathscr{E}}$ "differ by a compact operator". By Lemma 3.19 of [Par09a], $\left(E, T^{\mathscr{E}}\right)$ is a $\mathrm{KK}^{\mathrm{ban}}$-cycle and homotopic to $(E, T)$.

We have a similar result for homotopies: If $\left(E_{0}, T_{0}\right)$ and $\left(E_{1}, T_{1}\right)$ are homotopic in $\mathbb{E}_{g}^{\mathrm{ban}}(A, B)$ and if $T_{0}$ and $T_{1}$ are equivariant, then there is an equivariant homotopy between them.

This shows that the map $(E, T) \mapsto\left(E, T^{\mathscr{E}}\right)$ is bijective on the level of $\mathrm{KK}_{\mathscr{G}}^{\mathrm{ban}}$ classes. 
3.4. Monotone completions. In [Laf02] and [Laf06], the notion of an unconditional completion was introduced which is a special case of what we propose to call a monotone completion. Already the article [Laf02] provides us with some interesting examples of monotone completions which are not unconditional completions. ${ }^{5}$ The difference simply is that an unconditional completion is required to carry a product making it a Banach algebra whereas an unconditional completion is a Banach space without any product.

Let $Y$ be a locally compact Hausdorff space.

Definition 3.5. A semi-norm $\|\cdot\|_{\mathscr{H}}$ on $\mathcal{C}_{c}(Y)$ is called monotone if, for all $\varphi_{1}, \varphi_{2} \in$ $\mathcal{C}_{c}(Y)$, the following condition holds:

$$
\left.\left|\varphi_{1}(y)\right| \leq\left|\varphi_{2}(y)\right|\right) \Longrightarrow\left\|\varphi_{1}\right\|_{\mathscr{H}} \leq\left\|\varphi_{2}\right\|_{\mathscr{H}} \quad \text { for all } y \in Y .
$$

Let $\mathscr{H}(Y)$ denote the (Hausdorff-)completion of $\mathcal{C}_{c}(Y)$ with respect to this seminorm; this Banach space is called a monotone completion of $\mathcal{C}_{c}(Y)$.

For the rest of this section, let $\mathscr{H}(Y)$ be a monotone completion of $\mathcal{C}_{c}(Y)$.

For technical reasons and as for unconditional norms, we extend monotone norms to a larger class of functions on $Y$ :

Definition 3.6. Let $\mathscr{F}_{c}(Y)$ be the set of all (locally) bounded functions $\varphi: Y \rightarrow \mathbb{R}$ with compact support. Let $\mathcal{F}_{c}^{+}(Y)$ be the set of elements of $\mathscr{F}_{c}(Y)$ which are nonnegative. Define

$$
\|\varphi\|_{\mathscr{H}}:=\inf \left\{\|\psi\|_{\mathscr{H}}: \psi \in \mathcal{C}_{c}(Y), \psi \geq \varphi\right\}
$$

for all $\varphi \in{\mathscr{F}_{c}^{+}}_{c}^{+}(Y)$.

Note that, by property (4), the new semi-norm agrees on $\mathcal{C}_{c}^{+}(Y)$ with the seminorm we started with. For all $\varphi_{1}, \varphi_{2}, \varphi \in{F_{F}}_{c}^{+}(Y)$ and all $c \geq 0$, we have

(1) $\varphi_{1}+\varphi_{2} \in{F_{F}}_{c}^{+}(Y)$ and $\left\|\varphi_{1}+\varphi_{2}\right\| H_{\mathcal{H}} \leq\left\|\varphi_{1}\right\|_{\mathscr{H}}+\left\|\varphi_{2}\right\|_{\mathscr{H}}$;

(2) $c \varphi \in \mathscr{F}_{c}^{+}(Y)$ and $\|c \varphi\|_{\mathscr{H}}=c\|\varphi\|_{\mathscr{H}}$;

(3) if $\varphi_{1} \leq \varphi_{2}$, then $\left\|\varphi_{1}\right\|_{\mathscr{H}} \leq\left\|\varphi_{2}\right\|_{\mathscr{H}}$.

Hence we can use the extended semi-norm to define a semi-norm on sections of u.s.c. fields of Banach spaces.

For the rest of this section, let $E$ be a u.s.c. field of Banach spaces over $Y$.

Definition 3.7. We define the following semi-norm on $\Gamma_{c}(Y, E)$ :

$$
\|\xi\|_{\mathscr{H}}:=\|y \mapsto\| \xi(y)\left\|_{E_{y}}\right\|_{\mathscr{H}} .
$$

The Hausdorff completion of $\Gamma_{c}(Y, E)$ with respect to this semi-norm will be denoted by $\mathscr{H}(Y, E)$.

\footnotetext{
${ }^{5}$ For example $H^{2}(G, A)$ defined after Lemme 1.6.5 or the "normalised" completions $\mathrm{L}_{\text {norm }}^{p, l}(G, A)$ appearing in 4.5 .
} 
Note that the function $y \mapsto\|\xi(y)\|$ appearing in the preceding definition is not necessarily continuous. However, it has compact support and is non-negative upper semi-continuous, so we can apply the extended semi-norm on $\mathscr{F}_{c}^{+}(Y)$ to it.

If $E$ is the trivial bundle over $Y$ with fibre $E_{0}$, then $\Gamma_{c}(Y, E)$ is $\mathcal{C}_{c}\left(Y, E_{0}\right)$. The completion $\mathscr{H}(Y, E)$ of $\mathcal{C}_{c}\left(Y, E_{0}\right)$ could hence also be denoted as $\mathscr{H}\left(Y, E_{0}\right)$ and might be considered as a sort of tensor product of $\mathscr{H}(Y)$ and $E_{0}$. If in particular $E_{0}=\mathbb{C}$, then $\mathscr{H}(Y, E)=\mathscr{H}(Y, \mathbb{C})=\mathscr{H}(Y)$.

Definition 3.8. Let $F$ be another u.s.c. field of Banach spaces over $Y$ and let $T$ be a bounded continuous field of linear maps from $E$ to $F$. Then $\xi \mapsto T \circ \xi$ is a linear map from $\Gamma_{c}(Y, E)$ to $\Gamma_{c}(Y, F)$ such that $\|T \circ \xi\|_{\mathscr{H}} \leq\|T\|\|\xi\|_{\mathscr{H}}$. Hence $T$ induces a canonical continuous linear map from $\mathscr{H}(Y, E)$ to $\mathscr{H}(Y, F)$ with norm $\leq\|T\|$.

This way, we define a functor from the category of u.s.c. fields of Banach spaces over $Y$ to the category of Banach spaces, which is linear and contractive on the morphism sets.

Note that the canonical map from $\Gamma_{c}(Y, E)$ to $\mathscr{H}(Y, E)$ is continuous if we take the inductive limit topology on $\Gamma_{c}(Y, E)$ and the norm topology on $\mathscr{H}(Y, E)$. It follows that if a subset of $\Gamma_{c}(Y, E)$ is dense in $\Gamma_{c}(Y, E)$ for the inductive limit topology, then its canonical image in $\mathscr{H}(Y, E)$ is dense for the norm topology.

\section{The proof}

In this section, let $\mathscr{E}$ be a locally compact Hausdorff groupoid with unit space $X$ and carrying a Haar system $\lambda$.

4.1. The homomorphism $\boldsymbol{J}_{\mathcal{A}}^{\boldsymbol{B}}$. In Section 4.1, assume that $\mathscr{E}$ is proper and admits a cut-off function.

\subsubsection{The algebraic construction of $J_{\mathcal{A}}^{B}$ on the level of sections with compact} support. Let $E$ be a $\mathscr{G}$-Banach $B$-pair. Define the operations

and

$$
\left(e^{>} \beta\right)(x):=\int_{\mathscr{G} x} \gamma e^{>}(s(\gamma)) \gamma \beta\left(\gamma^{-1}\right) \mathrm{d} \lambda^{x}(\gamma)
$$

$$
\left(\beta e^{<}\right)(x):=\int_{\mathscr{G} x} \beta(\gamma) \gamma e^{<}(s(\gamma)) \mathrm{d} \lambda^{x}(\gamma),
$$

where $x \in X$, and the $\Gamma_{c}\left(\mathscr{E}, r^{*} B\right)$-valued bracket

$$
\left\langle\left\langle e^{<}, e^{>}\right\rangle\right\rangle(\gamma):=\left\langle e^{<}(r(\gamma)), \gamma e^{>}(s(\gamma))\right\rangle_{E_{r(\gamma)}},
$$

where $\gamma \in \mathcal{G}$, for all $e^{<} \in \Gamma_{c}\left(X, E^{<}\right), e^{>} \in \Gamma_{c}\left(X, E^{>}\right)$and $\beta \in \Gamma_{c}\left(\mathcal{G}, r^{*} B\right)$. 
This turns $\Gamma_{c}\left(X, E^{>}\right)$into a right $\Gamma_{c}\left(\mathscr{E}, r^{*} B\right)$-module and $\Gamma_{c}\left(X, E^{<}\right)$into a left $\Gamma_{c}\left(\mathcal{E}, r^{*} B\right)$-module. These module actions are separately continuous, and they are non-degenerate for the inductive limit topologies if $E$ is non-degenerate. The bracket is $\mathbb{C}$-bilinear and $\Gamma_{c}\left(\mathscr{E}, r^{*} B\right)$-linear on the left and on the right. Moreover, it is separately continuous for the inductive limit topologies.

Moreover, there are canonical actions of $\mathcal{C}(X / \mathscr{E})$ on the modules $\Gamma_{c}\left(X, E^{<}\right)$and $\Gamma_{c}\left(X, E^{>}\right)$given by

$$
\left(\chi e^{>}\right)(x):=\chi(\pi(x)) e^{>}(x)
$$

for all $\chi \in \mathcal{C}(X / \mathscr{E}), e^{>} \in \Gamma_{c}\left(X, E^{>}\right)$and $x \in X$ (and analogously for the left-hand side). The module actions and the bracket are compatible with these actions.

Let $E$ and $F$ be $\mathcal{E}$-Banach $B$-pairs and let $T$ be a $\mathscr{E}$-equivariant continuous field of operators from $E$ to $F$. For all $e^{>} \in \Gamma_{c}\left(X, E^{>}\right)$, define

$$
\left(\Gamma_{c}\left(X, T^{>}\right) e^{>}\right)(x):=T_{x}^{>}\left(e^{>}(x)\right)
$$

for all $x \in X$. Then $e^{>} \mapsto \Gamma_{c}\left(X, T^{>}\right) e^{>}$is $\mathbb{C}$-linear, $\mathcal{C}(X / \mathcal{E})$-linear, $\Gamma_{c}\left(\mathcal{E}, r^{*} B\right)$ linear on the right and continuous for the inductive limit topology. The same formula defines a similar map $f^{<} \mapsto \Gamma_{c}\left(X, T^{<}\right) f^{<}$on the left-hand side. The pair $\left(f^{<} \mapsto\right.$ $\left.\Gamma_{c}\left(X, T^{<}\right) f^{<}, e^{>} \mapsto \Gamma_{c}\left(X, T^{>}\right) e^{>}\right)$of linear operators is formally adjoint with respect to the brackets on $\left(\Gamma_{c}\left(X, E^{<}\right), \Gamma_{c}\left(X, E^{>}\right)\right)$and $\left(\Gamma_{c}\left(X, F^{<}\right), \Gamma_{c}\left(X, F^{>}\right)\right)$:

$$
\left\langle\left\langle f^{<} \Gamma_{c}\left(X, T^{<}\right), e^{>}\right\rangle\right\rangle=\left\langle\left\langle f^{<}, \Gamma_{c}\left(X, T^{>}\right) e^{>}\right\rangle\right\rangle .
$$

4.1.2. The analytic part of the construction of $\boldsymbol{J}_{\mathcal{A}}^{\boldsymbol{B}}$. In the $\mathrm{C}^{*}$-world, the right module $\Gamma_{c}\left(\mathcal{E}, r^{*} B\right)$-action and the inner product on $\Gamma_{c}(X, E)$ is sufficient to define the structure $B \rtimes_{r} \mathscr{G}$-Hilbert module if $E$ is a Hilbert $B$-module. There can only be one norm on $\Gamma_{c}(X, E)$ which completes to a Hilbert module and the bracket actually gives such a norm.

In the Banach-world, the situation is more complicated. If $B$ is a $\mathscr{E}$-Banach algebra and $E$ is a $\mathscr{E}$-Banach $B$-pair, then we will see that there are several ways to complete $\Gamma_{c}\left(X, E^{<}\right)$and $\Gamma_{c}\left(X, E^{>}\right)$to give a $\mathcal{C}_{0}(X / \mathscr{G})$-Banach $\mathcal{A}(\mathcal{E}, B)$-pair. However, it turns out that every (monotone) pair of such completions will give rise to the same homomorphism $J_{\mathscr{A}}^{B}$.

Let $\mathscr{D}^{<}(X)$ and $\mathscr{D}^{>}(X)$ be monotone completions of $\ell_{c}(X)$. Assume that the pair $\mathscr{D}(X):=\left(\mathscr{D}^{<}(X), \mathscr{D}^{>}(X)\right)$ satisfies the following compatibility conditions with $\mathcal{A}(\mathscr{E})$ :

(D1) $\left\|\beta \chi^{<}\right\|_{\mathscr{D}^{<}} \leq\|\beta\|_{\mathscr{A}}\left\|\chi^{<}\right\|_{\mathscr{D}^{<}}$for all $\chi^{<} \in \mathscr{C}_{c}(X), \beta \in \mathcal{C}_{c}(\mathscr{G})$, and $\left\|\chi^{>} \beta\right\|_{D^{>}} \leq\left\|\chi^{>}\right\|_{D^{>}}\|\beta\|_{\mathscr{A}}$ for all $\chi^{>} \in \mathscr{C}_{c}(X), \beta \in \mathscr{C}_{c}(\mathscr{G})$.

(D2) $\|\left\langle\left\langle\chi^{<}, \chi^{>}\right\rangle\left\|_{\mathcal{A}} \leq\right\| \chi^{<}\left\|_{\mathscr{D}^{<}}\right\| \chi^{>} \|_{D^{>}}\right.$for all $\chi^{<} \in \mathcal{C}_{c}(X), \chi^{>} \in \mathcal{C}_{c}(X)$.

We can extend the actions of $\mathcal{C}_{c}(\mathscr{E})$ on $\mathcal{C}_{c}(X)$ from the left and from the right and also the inner product to continuous bilinear maps which turn $\mathscr{D}(X)$ into a Banach 
$\mathcal{A}(\mathscr{E})$-pair. Note that the action of $\mathcal{C}_{0}(X / \mathscr{E})$ on $\mathcal{C}_{c}(X)$ also gives a continuous nondegenerate action of $\mathcal{C}_{0}(X / \mathscr{E})$ on $\mathscr{D}^{<}(X)$ and $\mathscr{D}^{>}(X)$ making $\mathscr{D}(X)$ a $\mathcal{C}_{0}(X / \mathscr{E})$ Banach $\mathcal{A}(\mathscr{E})$-pair.

Let $E=\left(E^{<}, E^{>}\right)$be a $\mathscr{G}$-Banach $B$-pair. On $\Gamma_{c}\left(X, E^{<}\right)$define $\left\|\xi^{<}\right\|_{D^{<}}:=$ $\|x \mapsto\| \xi^{<}(x)\|\|_{D^{<}}$as in Definition 3.7 and define a semi-norm $\|\cdot\|_{D^{>}}$on $\Gamma_{c}\left(X, E^{>}\right)$ similarly. Then the actions of $\Gamma_{c}\left(\mathcal{G}, r^{*} B\right)$ on $\Gamma_{c}\left(X, E^{<}\right)$and on $\Gamma_{c}\left(X, E^{>}\right)$and the bracket satisfy

$$
\begin{aligned}
\left\|\beta \xi^{<}\right\|_{D^{<}} & \leq\|\beta\|_{\mathscr{A}}\left\|\xi^{<}\right\|_{D^{<}}, \\
\left\|\xi^{>} \beta\right\|_{D^{>}} & \leq\left\|\xi^{>}\right\|_{D^{>}}\|\beta\|_{\mathscr{A}}, \\
\left.\|\left\langle\xi^{<}, \xi^{>}\right\rangle\right\rangle \|_{\mathscr{A}} & \leq\left\|\xi^{<}\right\|_{D^{<}}\left\|\xi^{>}\right\|_{\mathscr{D}^{>}}
\end{aligned}
$$

for all $\beta \in \Gamma_{c}\left(\mathscr{G}, r^{*} B\right), \xi^{<} \in \Gamma_{c}\left(X, E^{<}\right)$and $\xi^{>} \in \Gamma_{c}\left(X, E^{>}\right)$. As in Definition 3.7, write $\mathscr{D}^{<}\left(X, E^{<}\right)$for the completion of $\Gamma_{c}\left(X, E^{<}\right)$for the semi-norm $\|\cdot\|_{D^{<}}$; define $\mathscr{D}^{>}\left(X, E^{>}\right)$analogously. With the extensions of the actions of $\Gamma_{c}\left(\mathcal{G}, r^{*} B\right)$ and the extension of the bracket,

$$
\mathscr{D}(X, E):=\left(\mathscr{D}^{<}\left(X, E^{<}\right), \mathscr{D}^{>}\left(X, E^{>}\right)\right)
$$

is a $\mathscr{C}_{0}(X / \mathscr{G})$-Banach $\mathcal{A}(\mathscr{E}, B)$-pair.

If $F$ is another $\mathscr{E}$-Banach $B$-pair and $T \in \mathrm{L}_{B}(E, F)$ is $\mathscr{G}$-equivariant, then $\Gamma_{c}\left(X, T^{>}\right)$is a bounded linear map from $\Gamma_{c}\left(X, E^{>}\right)$to $\Gamma_{c}\left(X, F^{>}\right)$with norm less than or equal to $\left\|T^{>}\right\|$, so it extends to a bounded $\mathbb{C}$-linear, $\ell_{0}(X / \mathscr{E})$-linear and $\mathcal{A}(\mathscr{E}, B)$-linear map $\mathscr{D}\left(X, T^{>}\right)$from $\mathscr{D}\left(X, E^{>}\right)$to $\mathscr{D}\left(X, F^{>}\right)$of the same norm. Similarly, one gets a bounded linear map $\mathscr{D}\left(X, T^{<}\right)$from $\mathscr{D}\left(X, F^{<}\right)$to $\mathscr{D}\left(X, E^{<}\right)$. Together, this defines a linear operator

$$
\mathscr{D}(X, T):=\left(\mathscr{D}\left(X, T^{<}\right), \mathscr{D}\left(X, T^{>}\right)\right) \in \mathrm{L}_{\mathcal{A}(\mathscr{E}, B)}^{\mathcal{\ell}_{0}(X / \mathscr{E})}(\mathscr{D}(X, E), \mathscr{D}(X, F))
$$

of norm less than or equal to $\|T\|$. The assignment $E \mapsto \mathscr{D}(X, E)$ and $T \mapsto \mathscr{D}(X, T)$ is a contractive functor from the category of $\mathscr{E}$-Banach $B$-pairs and bounded $\mathscr{E}$ equivariant operators to the category of $\mathcal{C}_{0}(X / \mathscr{E})$-Banach $\mathcal{A}(\mathscr{E}, B)$-pairs. Similarly, one can define $\mathscr{D}(X, \Phi)$ for $\mathcal{G}$-equivariant concurrent homomorphisms.

We omit the longsome proof of the following result which can be found in [Par07], Section 7.2.3:

Proposition 4.1. Let $S \in \mathrm{L}_{B}(E, F)$ be bounded, $\mathcal{G}$-equivariant and locally compact. Then $\mathscr{D}(X, S)$ is locally compact in the sense of Definition 1.4, i.e., $\chi \mathscr{D}(X, S)$ is compact for all $\chi \in \mathcal{C}_{c}(X / \mathcal{G})$.

\subsubsection{The construction for $\mathrm{KK}^{\mathrm{ban}}$-cycles}

Theorem 4.2. Let $(E, T)$ be a cycle in $\mathbb{E}_{g}^{\mathrm{ban}}\left(\mathcal{C}_{0}(X), B\right)$. We assume that $T$ is $\mathcal{G}$-equivariant, compare Proposition 3.4. Equip $\mathscr{D}(X, E)$ with the obvious grading operator. Then $\mathscr{D}(X, T)$ is odd and

$$
J_{\mathcal{A}, \mathcal{D}}^{B}(E, T):=(\mathscr{D}(X, E), \mathscr{D}(X, T)) \in \mathbb{E}^{\mathrm{ban}}\left(\mathcal{C}_{0}(X / \mathcal{G}) ; \mathcal{C}_{0}(X / \mathcal{E}), \mathcal{A}(\mathcal{E}, B)\right) .
$$


Proof. The important property that we have to check is that $\mathscr{D}(X, T)^{2}-1$ is locally compact. But

$$
\mathscr{D}(X, T)^{2}-1=\mathscr{D}\left(X, T^{2}-1\right),
$$

and $T^{2}-1$ is locally compact. Since $T^{2}-1$ is also $\mathscr{E}$-equivariant, we can apply Proposition 4.1 which implies that $\mathscr{D}\left(X, T^{2}-1\right)$ is locally compact.

It can be shown that the map $J_{\mathscr{A}, \mathscr{D}}^{B}$ is compatible with the pushforward along equivariant homomorphisms of $\mathcal{G}$-Banach algebras, with homotopies and with the sum of cycles; see [Par07], Section 7.2.3, for the proofs.

As a consequence of these results, we have:

Proposition 4.3. The map $(E, T) \mapsto(\mathscr{D}(X, E), \mathscr{D}(X, T))$ gives rise to a group homomorphism

$$
J_{\mathcal{A}, \mathcal{D}}^{B}: \operatorname{KK}_{\mathscr{G}}^{\mathrm{ban}}\left(\mathcal{C}_{0}(X), B\right) \rightarrow \mathcal{R} \mathrm{KK}^{\mathrm{ban}}\left(\mathcal{C}_{0}(X / \mathscr{E}) ; \mathcal{C}_{0}(X / \mathscr{E}), \mathcal{A}(\mathscr{E}, B)\right)
$$

which is natural in the non-degenerate $\mathscr{E}$-Banach algebra $B$.

4.1.4. Uniqueness and existence. The following uniqueness result was shown in [Par07]:

Definition and Proposition 4.4. Let $\mathscr{D}(X)$ and $\mathscr{D}^{\prime}(X)$ be pairs of monotone completions of $\mathcal{C}_{c}(X)$ which both satisfy (D1) and (D2). Then $J_{\mathcal{A}, \mathscr{D}}^{B}=J_{\mathscr{A}, \mathcal{D}^{\prime}}^{B}$ as homomorphisms from $\mathrm{KK}_{\mathscr{G}}^{\mathrm{ban}}\left(\mathcal{C}_{0}(X), B\right)$ to $\mathcal{R} \mathrm{KK}^{\mathrm{ban}}\left(\mathcal{C}_{0}(X / \mathscr{G}) ; \mathcal{C}_{0}(X / \mathcal{G}), \mathcal{A}(\mathcal{G}, B)\right)$. We hence write $J_{\mathscr{A}}^{B}$ for this homomorphism.

Another question is whether such pairs $\mathscr{D}(X)$ of monotone completions exist. We have a positive answer because we have assumed that $\mathcal{E}$ admits a cut-off function; there are even quite a few such completions: for every cut-off pair $c$, we construct a compatible pair of monotone completions that we call $\mathscr{A}^{c}(X)$.

So let $c=\left(c^{<}, c^{>}\right)$be a cut-off pair for $\mathscr{E}$. Let $E$ be a $\mathscr{E}$-Banach $B$-pair. Define

$$
j_{E, c}^{<}: \Gamma_{c}\left(X, E^{<}\right) \rightarrow \Gamma_{c}\left(\mathcal{E}, E^{<}\right), \quad e^{<} \mapsto\left(\gamma \mapsto c^{<}(s(\gamma)) e^{<}(r(\gamma))\right)
$$

and

$$
j_{E, c}^{>}: \Gamma_{c}\left(X, E^{>}\right) \rightarrow \Gamma_{c}\left(\mathcal{G}, E^{>}\right), \quad e^{>} \mapsto\left(\gamma \mapsto c^{>}(r(\gamma)) \gamma e^{>}(s(\gamma))\right) .
$$

Then $j_{E, c}=\left(j_{E, c}^{<}, j_{E, c}^{>}\right)$is a pair of injective maps such that

(1) $j_{E, c}^{<}$is $\mathbb{C}$-linear, $\Gamma_{c}(X / \mathscr{E})$-linear and $\Gamma_{c}\left(\mathscr{E}, r^{*} B\right)$-linear on the left,

(2) $j_{E, c}^{>}$is $\mathbb{C}$-linear, $\Gamma_{c}(X / \mathscr{G})$-linear and $\Gamma_{c}\left(\mathscr{G}, r^{*} B\right)$-linear on the right,

(3) for all $e^{<} \in \Gamma_{c}\left(X, E^{<}\right)$and $e^{>} \in \Gamma_{c}\left(X, E^{>}\right)$, we have

$$
\left\langle j_{E, c}^{<}\left(e^{<}\right), j_{E, c}^{>}\left(e^{>}\right)\right\rangle_{\Gamma_{c}\left(\mathscr{\xi}, r^{*} B\right)}=\left\langle\left\langle e^{<}, e^{>}\right\rangle\right\rangle .
$$


Define a $\mathscr{C}_{0}(X / \mathscr{E})$-Banach $\mathcal{A}(\mathscr{E}, B)$-pair $\mathcal{A}^{c}(X, E)=\left(\mathcal{A}^{c}\left(X, E^{<}\right), \mathcal{A}^{c}\left(X, E^{>}\right)\right)$ by pulling back the norms of $\mathcal{A}(\mathscr{G}, E)$ along $j_{E, c}$ and completing $\Gamma_{c}(X, E)$ for this norms. Alternatively, one could take the closure of the image of $j_{E, c}$. The norms on the left and the right part are given by

$$
\left\|e^{<}\right\|_{\mathcal{A}^{c}\left(X, E^{<}\right)}:=\left\|j_{E, c}^{<}\left(e^{<}\right)\right\|_{\mathcal{A}\left(\mathscr{E}, E^{<}\right)}=\left\|\gamma \mapsto c^{<}(s(\gamma))\right\| e^{<}(r(\gamma))\|\|_{\mathcal{A}}
$$

and

$$
\left\|e^{>}\right\|_{\mathcal{A}^{c}\left(X, E^{>}\right)}:=\left\|j_{E, c}^{>}\left(e^{>}\right)\right\|_{\mathcal{A}\left(\mathcal{G}, E^{>}\right)}=\left\|\gamma \mapsto c^{>}(r(\gamma))\right\| e^{>}(s(\gamma))\|\|_{\mathcal{A}}
$$

for all $e^{<} \in \Gamma_{c}\left(X, E^{<}\right)$and $e^{>} \in \Gamma_{c}\left(X, E^{>}\right)$.

Note that the norms depend on $\mathcal{A}(\mathscr{E})$ as well as on $c$. The pair $\mathcal{A}^{c}(X)=$ $\left(\left(\mathcal{A}^{c}\right)^{<}(X),\left(\mathcal{A}^{c}\right)^{>}(X)\right)$ is a pair of monotone completions of $\mathcal{C}_{c}(X)$ satisfying (D1) and (D2). Note that $J_{\mathscr{A}, \mathcal{A}^{c}}^{B}$ as a homomorphism from $\mathrm{KK}_{\mathscr{g}}^{\mathrm{ban}}\left(\mathcal{C}_{0}(X), B\right)$ to $\mathcal{R K K}^{\text {ban }}\left(\mathcal{C}_{0}(X / \mathscr{E}) ; \mathcal{C}_{0}(X / \mathscr{G}), \mathcal{A}(\mathscr{E}, B)\right)$ does not depend on $c$ by 4.4 ; without the detour via more general compatible pairs $\mathscr{D}(X)$ of monotone completions this latter fact seems to be hard to prove.

4.2. Monotone completions as analogues of $\mathrm{L}^{2}(\mathcal{E}, B)$. If $B$ is a $\mathscr{E}$ - $\mathrm{C}^{*}$-algebra, then there is a canonical $\mathscr{E}$-Hilbert $B$-module $\mathrm{L}^{2}(\mathscr{E}, B)$ with a left action of $B \rtimes_{r} \mathscr{E}$. We want to find an analogue of this module for the case that $B$ is a general $\mathscr{G}$-Banach algebra. Apparently, it is not sufficient (or not systematic, at least) to just consider pairs of the type $\left(\mathrm{L}^{2}(\mathcal{G}, B), \mathrm{L}^{2}(\mathcal{E}, B)\right)$; we want to treat rather general unconditional completions, so it seems appropriate to consider rather general completions of the space $\Gamma_{c}\left(\mathcal{G}, r^{*} B\right)$ as substitutes of $\mathrm{L}^{2}(\mathcal{E}, B)$; our treatment should at least cover pairs of the form $\left(\mathrm{L}^{1}(\mathcal{E}, B), \Gamma_{0}(\mathscr{E}, B)\right)$ or $\left(\mathrm{L}^{p^{\prime}}(\mathcal{E}, B), \mathrm{L}^{p}(\mathcal{E}, B)\right)$ for $\left.p, p^{\prime} \in\right] 1, \infty[$ with $1 / p+1 / p^{\prime}=1$ (compare the precise definitions below).

Our substitute for $\mathrm{L}^{2}(\mathscr{E})$ is a general pair of monotone completions of $\mathcal{C}_{c}(\mathscr{E})$ which satisfies some compatibility conditions with $\mathcal{A}(\mathscr{G})$ and the action of $\mathscr{G}$; we will usually denote such a pair by $\mathscr{H}(\mathscr{E})$, and write $\mathscr{H}(\mathscr{E}, B)$ for its version with coefficients in $B$. It seems advisable to even consider pairs of the form $\mathscr{H}(\mathscr{G}, E)$ where $E$ is a $\mathscr{G}$-Banach $B$-pair because this makes the constructions a bit clearer. The important result is that (under certain conditions) the unconditional completion $\mathcal{A}(\mathscr{E}, B)$ acts on $\mathscr{H}(\mathscr{E}, B)$ by locally compact operators. This allows us to use the tensor product $\otimes_{\mathcal{A}(\mathscr{E}, B)} \mathscr{H}(\mathscr{G}, B)$ to turn $\mathcal{A}(\mathscr{E}, B)$-pairs into $\mathscr{E}$-Banach $B$-pairs preserving locally compact operators between them.

Recall that, in this section, $\boldsymbol{E}$ denotes a locally compact Hausdorff groupoid with left Haar system $\lambda$ and $X$ denotes the unit space of $\mathscr{E}$. Recall also that $\mathcal{A}(\mathscr{E})$ is an unconditional completion of $\mathcal{C}_{c}(\mathcal{E})$ and that $B$ is a non-degenerate $\mathcal{E}$-Banach algebra.

Let $\mathscr{H}(\mathscr{G})=\left(\mathscr{H}^{<}(\mathscr{G}), \mathscr{H}^{>}(\mathscr{G})\right)$ be a pair of monotone completions of $\mathcal{C}_{c}(\mathscr{G})$ 
such that the bilinear map

$$
\begin{aligned}
\langle\cdot, \cdot\rangle e_{c}(X): e_{c}(\mathscr{E}) \times e_{c}(\mathscr{E}) & \rightarrow e_{c}(X), \\
\left(\varphi^{<}, \varphi^{>}\right) & \mapsto\left(x \mapsto \int_{\mathscr{E} x} \varphi^{<}(\gamma) \varphi^{>}\left(\gamma^{-1}\right) \mathrm{d} \lambda^{x}(\gamma)\right),
\end{aligned}
$$

satisfies

(H1) $\left\|\left\langle\varphi^{<}, \varphi^{>}\right\rangle e_{c}(X)\right\|_{\infty} \leq\left\|\varphi^{<}\right\|_{\mathscr{H}^{<}}\left\|\varphi^{>}\right\|_{\mathscr{H}^{>}}$for all $\varphi^{<}, \varphi^{>} \in \boldsymbol{C}_{c}(\mathscr{E})$.

In this case, $\langle\cdot, \cdot\rangle \epsilon_{c}(X)$ can be extended to a continuous bilinear map $\langle\cdot, \cdot\rangle_{\ell_{0}(X)}: \mathscr{H}^{<}(\mathscr{E}) \times \mathscr{H}^{>}(\mathscr{E}) \rightarrow \boldsymbol{C}_{0}(X)$ which is $\boldsymbol{C}_{0}(X)$-bilinear if we consider the following actions of $\varphi_{0}(X)$ :

$$
\left(\chi \xi^{<}\right)(\gamma):=\chi(r(\gamma)) \xi^{<}(\gamma) \text { and }\left(\xi^{>} \chi\right)(\gamma):=\xi^{>}(\gamma) \chi(s(\gamma))
$$

for all $\chi \in \mathcal{C}_{0}(X), \xi^{<} \in \mathcal{C}_{c}(\mathscr{G}) \subseteq \mathscr{H}^{<}(\mathscr{G}), \xi^{>} \in \mathcal{C}_{c}(\mathscr{E}) \subseteq \mathscr{H}^{>}(\mathscr{E})$ and $\gamma \in \mathcal{E}$

Examples 4.5. Let $p \in[1, \infty[$. Define the norm

$$
\left\|\chi^{<}\right\|_{p, r}:=\sup _{x \in X}\left(\int_{\mathscr{E} x}\left|\chi^{<}(\gamma)\right|^{p} \mathrm{~d} \lambda^{x}(\gamma)\right)^{\frac{1}{p}}
$$

for all $\chi^{<} \in \boldsymbol{C}_{c}(\mathscr{G})$. The corresponding monotone completion is called $\mathrm{L}_{r}^{p}(\mathcal{G})$. Note that $\mathrm{L}^{1}(\mathscr{\mathscr { C }})=\mathrm{L}_{r}^{1}(\mathscr{G})$. Secondly, define

$$
\left\|\chi^{>}\right\|_{p, s}:=\sup _{x \in X}\left(\int_{g_{x}}\left|\chi^{>}\left(\gamma^{-1}\right)\right|^{p} \mathrm{~d} \lambda^{x}(\gamma)\right)^{\frac{1}{p}}
$$

for all $\chi^{>} \in \mathcal{C}_{c}(\mathscr{G})$. The corresponding monotone completion is called $\mathrm{L}_{s}^{p}(\mathcal{G})$.

(1) The pairs $\left(\mathrm{L}^{1}(\mathscr{E}), \mathcal{C}_{0}(\mathscr{E})\right)$ and $\left(\mathcal{C}_{0}(\mathscr{E}), \mathrm{L}_{s}^{1}(\mathscr{E})\right)$ are pairs of monotone completions of $\mathcal{C}_{c}(\mathscr{G})$ satisfying $(\mathrm{H} 1)$.

(2) If $\left.p, p^{\prime} \in\right] 1, \infty\left[\right.$ such that $\frac{1}{p}+\frac{1}{p^{\prime}}=1$, then $\left(\mathrm{L}_{r}^{p^{\prime}}(\mathscr{G}), \mathrm{L}_{s}^{p}(\mathscr{E})\right)$ also satisfies $(\mathrm{H} 1)$.

(3) In particular, this applies to $\left(\mathrm{L}_{r}^{2}(\mathscr{E}), \mathrm{L}_{s}^{2}(\mathscr{G})\right)$.

Now let $E$ be a $\mathcal{E}$-Banach $B$-pair. Define a right action of $\Gamma(X, B)$ on $\Gamma_{c}\left(\mathcal{E}, r^{*} E^{>}\right)$ by

$$
\left(\xi^{>} \beta\right)(\gamma):=\xi^{>}(\gamma) \gamma \beta(s(\gamma)), \quad \xi^{>} \in \Gamma_{c}\left(\mathcal{G}, r^{*} E^{>}\right), \beta \in \Gamma(X, B), \gamma \in \mathcal{E},
$$

and a left action of $\Gamma(X, B)$ on $\Gamma_{c}\left(\mathcal{G}, r^{*} E^{<}\right)$by

$$
\left(\beta \xi^{<}\right)(\gamma):=\beta(r(\gamma)) \xi^{<}(\gamma), \quad \beta \in \Gamma(X, B), \xi^{<} \in \Gamma_{c}\left(\mathcal{E}, r^{*} E^{<}\right), \gamma \in \mathcal{E} .
$$


These actions define continuous actions of $\Gamma_{0}(X, B)$ on $\mathscr{H}^{>}\left(\mathscr{E}, E^{>}\right)$(from the right) and $\mathscr{H}^{<}\left(\mathscr{G}, E^{<}\right)$(from the left). Define a bilinear map $\langle\cdot, \cdot\rangle_{\Gamma_{c}(X, B)}$ :

$$
\begin{aligned}
\Gamma_{c}\left(\mathscr{E}, r^{*} E^{<}\right) \times \Gamma_{c}\left(\mathscr{E}, r^{*} E^{>}\right) & \rightarrow \Gamma_{c}(X, B), \\
\left(\xi^{<}, \xi^{>}\right) & \mapsto\left(x \mapsto \int_{\mathscr{E} x}\left\langle\xi^{<}(\gamma), \gamma \xi^{>}\left(\gamma^{-1}\right)\right\rangle_{E_{r(\gamma)}} \mathrm{d} \lambda^{x}(\gamma)\right) .
\end{aligned}
$$

This map extends to a contractive bracket from $\mathscr{H}^{<}\left(\mathscr{H}, E^{<}\right) \times \mathscr{H}^{>}\left(\mathscr{E}, E^{>}\right)$to $\Gamma_{0}(X, B)$ which makes $\mathscr{H}(\mathscr{G}, E):=\left(\mathscr{H}^{<}\left(\mathscr{E}, E^{<}\right), \mathscr{H}^{>}\left(\mathscr{E}, E^{>}\right)\right)$a $\mathcal{C}_{0}(X)$-Banach $\Gamma_{0}(X, B)$ pair. If $E$ is non-degenerate, then so is $\mathscr{H}(\mathscr{E}, E)$.

Note that the $\mathcal{C}_{0}(X)$-structures on $\mathscr{H}^{<}\left(\mathscr{E}, E^{<}\right)$and $\mathscr{H}^{>}\left(\mathscr{E}, E^{>}\right)$are not the same in general: on the left-hand side it is induced by the range map $r$, on the right-hand side by the source map $s$. This implies that the fibre of $\mathscr{H}^{<}\left(\mathscr{E}, E^{<}\right)$over some $x \in X$ should be regarded as a completion of $\Gamma_{c}\left(\mathscr{E}^{x}, E_{x}^{<}\right)$, whereas the fibre of $\mathscr{H}^{>}\left(\mathscr{E}, E^{>}\right)$ over $x$ should be regarded as a completion of $\Gamma_{c}\left(\mathscr{E}_{x},\left(r^{*} E^{>}\right) \mid g_{x}\right)$.

Assume now that $\mathscr{H}(\mathscr{E})$ has also the following properties:

(H2) $\left\|\xi^{<*} * \chi\right\|_{\mathscr{H}<(\mathscr{C})} \leq\left\|\xi^{<}\right\|_{\mathscr{H}<(\mathscr{G})}\|\chi\|_{\mathscr{A}(\mathscr{g})}$ for all $\chi, \xi^{<} \in \mathscr{C}_{c}(\mathscr{G})$, and $\left\|\chi * \xi^{>}\right\|_{\mathscr{H}>(\mathscr{E})} \leq\|\chi\|_{\mathcal{A}(\mathscr{\xi})}\left\|\xi^{>}\right\|_{\mathscr{H}>(\mathscr{E})}$ for all $\chi, \xi^{>} \in \mathcal{C}_{c}(\mathscr{E})$.

Let $A$ be another $\mathcal{E}$-Banach algebra and let $E$ be a $\mathscr{E}$-Banach $A$ - $B$-pair. For all $a \in \Gamma_{c}\left(\mathscr{G}, r^{*} A\right)$, all $\xi^{<} \in \Gamma_{c}\left(\mathscr{E}, r^{*} E^{<}\right)$and all $\xi^{>} \in \Gamma_{c}\left(\mathscr{G}, r^{*} E^{>}\right)$, define

$$
\left(a \xi^{>}\right)(\gamma)=\left(a * \xi^{>}\right)(\gamma)=\int_{\operatorname{gr}(\gamma)} a\left(\gamma^{\prime}\right) \gamma^{\prime} \xi^{>}\left(\gamma^{\prime-1} \gamma\right) \mathrm{d} \lambda^{r(\gamma)}\left(\gamma^{\prime}\right)
$$

and

$$
\left(\xi^{<} a\right)(\gamma)=\left(\xi^{<} * a\right)(\gamma)=\int_{\mathscr{g r}(\gamma)} \xi^{<}\left(\gamma^{\prime}\right) \gamma^{\prime} a\left(\gamma^{\prime-1} \gamma\right) \mathrm{d} \lambda^{r(\gamma)}\left(\gamma^{\prime}\right)
$$

for all $\gamma \in \mathscr{G}$. These actions lift to actions of $\mathcal{A}(\mathscr{E}, A)$ on $\mathscr{H}^{>}\left(\mathscr{E}, E^{>}\right)$and $\mathscr{H}^{<}\left(\mathscr{E}, E^{<}\right)$, respectively. Equipped with them, $\mathscr{H}(\mathscr{E}, E)$ becomes a $\mathcal{C}_{0}(X)$-Banach $\Gamma_{0}(X, B)$ pair on which $\mathcal{A}(\mathscr{G}, A)$ acts by elements of $\mathrm{L}_{\Gamma_{0}(X, B)}^{\mathcal{C}_{0}(X)}(\mathscr{H}(\mathscr{E}, E))$.

Proposition 4.6. If $\Gamma(X, A)$ acts on $E$ by locally compact operators and $\mathscr{E}$ is proper, then $\mathcal{A}(\mathcal{E}, A)$ acts on $\mathscr{H}(\mathcal{E}, E)$ by locally compact operators.

Proof. Let $a \in \Gamma_{c}\left(\mathscr{E}, r^{*} A\right)$ have compact support. If we can show that the action of $a$ on $\mathscr{H}(\mathcal{G}, E)$, denoted by $\pi(a) \in \mathrm{L}_{\Gamma_{0}(X, B)}(\mathscr{H}(\mathcal{E}, E))$, is locally compact, then we are done. Let $\chi \in \mathscr{\ell}_{c}(X)$. We have to show that $\chi \pi(a)$ is compact. It was shown in [Par07], Appendix E.8.3, that an operator which is given by a compact kernel with compact support is compact. We thus prove that $\chi \pi(a)$ is such an operator. Define

$$
k_{\left(\gamma_{1}, \gamma_{2}\right)}:=\chi\left(s\left(\gamma_{1}\right)\right) \pi_{A}\left(a\left(\gamma_{2}\right)\right) \in \mathrm{L}_{B_{r\left(\gamma_{1}\right)}}\left(E_{r\left(\gamma_{1}\right)}\right)
$$


for all $\left(\gamma_{1}, \gamma_{2}\right) \in \mathscr{E} *_{r, r} \mathscr{E}$. Then the action of $\chi \pi(a)$ on $\Gamma_{c}\left(\mathscr{E}, r^{*} E^{>}\right)$is given by

$$
\begin{aligned}
(\chi \pi(a))^{>}\left(\xi^{>}\right)(\gamma) & =\chi(s(\gamma)) \int_{\mathscr{g} r(\gamma)} a\left(\gamma^{\prime}\right) \gamma^{\prime} \xi^{>}\left(\gamma^{\prime-1} \gamma\right) \mathrm{d} \lambda^{r(\gamma)}\left(\gamma^{\prime}\right) \\
& =\int_{\mathscr{g} r(\gamma)} \chi(s(\gamma)) a\left(\gamma^{\prime}\right) \gamma^{\prime} \xi^{>}\left(\gamma^{\prime-1} \gamma\right) \mathrm{d} \lambda^{r(\gamma)}\left(\gamma^{\prime}\right) \\
& =\int_{\operatorname{gr}(\gamma)} k_{\left(\gamma, \gamma^{\prime}\right)} \gamma^{\prime} \xi^{>}\left(\gamma^{\prime-1} \gamma\right) \mathrm{d} \lambda^{r(\gamma)}\left(\gamma^{\prime}\right)
\end{aligned}
$$

for all $\xi^{>} \in \Gamma_{c}\left(\mathscr{E}, r^{*} E^{>}\right)$and $\gamma \in \mathscr{E}$. A similar calculation for the left-hand side shows that $\chi \pi(a)$ is indeed given by the kernel $k$.

The field of operators $\left(\pi_{A}\left(a\left(\gamma_{2}\right)\right)\right)_{\left(\gamma_{1}, \gamma_{2}\right) \in \mathcal{G}_{*_{r},} \mathscr{G}}$ is locally compact, so the same is true for $k$. Moreover, the support of $k$ is compact: Since $\mathscr{E}$ is proper, the set $K:=$ $\{\gamma \in \mathscr{G}: r(\gamma) \in \operatorname{supp} \chi, s(\gamma) \in r(\operatorname{supp} a)\}$ is compact. Let $\left(\gamma_{1}, \gamma_{2}\right) \in \mathscr{E} *_{r, r} \mathscr{E}$. Then $k_{\left(\gamma_{1}, \gamma_{2}\right)} \neq 0$ implies that $\gamma_{1} \in K$ and $\gamma_{2} \in \operatorname{supp} a$. So $\left(\gamma_{1}, \gamma_{2}\right)$ is contained in $K \times \operatorname{supp} a$. Hence $k$ has compact support.

As a corollary of Proposition 4.6 and because $B$ is non-degenerate, we get:

Corollary 4.7. If $\mathscr{G}$ is proper, then $\mathcal{A}(\mathcal{E}, B)$ acts on $\mathscr{H}(\mathscr{E}, B)$ by locally compact operators.

We now want to put an action of $\mathscr{E}$ on $\mathscr{H}(\mathscr{E}, E)$. Technically, we have to replace $\mathscr{H}(\mathscr{G}, E)$ with the u.s.c. field $\mathfrak{F}(\mathscr{H}(\mathscr{E}, E))$ of pairs over $X$, compare Section 1.3. So it is natural to assume:

(H3) The $\mathscr{C}_{0}(X)$-Banach space $\mathscr{H}^{<}(\mathscr{E})$ is locally $\mathscr{C}_{0}(X)$-convex with respect to the $\mathcal{C}_{0}(X)$-action induced by $r$ and $\mathscr{H}^{>}(\mathscr{G})$ is locally $\mathcal{C}_{0}(X)$-convex with respect to the action induced by $s$.

For all $\gamma \in \mathscr{E}$, define a map $\alpha_{\gamma}^{<}$from $\mathcal{C}_{c}\left(\mathscr{E}^{s(\gamma)}\right)$ to $\mathcal{C}_{c}\left(\mathscr{E}^{r(\gamma)}\right)$ by

$$
\chi^{<} \mapsto \alpha_{\gamma}^{<}\left(\chi^{<}\right)=\gamma \chi^{<}=\left(\gamma^{\prime} \mapsto \chi^{<}\left(\gamma^{-1} \gamma^{\prime}\right)\right)
$$

and a map $\alpha_{\gamma}^{>}$from $\mathscr{C}_{c}\left(\mathscr{E}_{s(\gamma)}\right)$ to $\mathcal{C}_{c}\left(\mathscr{E}_{r(\gamma)}\right)$ by

$$
\chi^{>} \mapsto \alpha_{\gamma}^{>}\left(\chi^{>}\right)=\gamma \chi^{>}=\left(\gamma^{\prime} \mapsto \chi^{>}\left(\gamma^{\prime} \gamma\right)\right) .
$$

To get an action of $\mathscr{E}$ on $\mathfrak{F}(\mathcal{H}(\mathscr{E}, E))$ we have to assume that $\alpha^{<}$and $\alpha^{>}$are families of isometric maps, i.e., if we have that

(H4) $\left\|\gamma \chi^{<}\right\|_{\mathscr{H}<(\mathscr{g} r(\gamma))}=\left\|\chi^{<}\right\|_{\mathscr{H}<(\mathcal{G} s(\gamma))}$ for all $\chi^{<} \in \mathcal{C}_{c}\left(\mathscr{G}^{s(\gamma)}\right)$ and all $\gamma \in \mathscr{E}$, and $\left\|\gamma \chi^{>}\right\|_{\mathscr{H}>\left(\mathscr{r}_{r(\gamma)}\right)}=\left\|\chi^{>}\right\|_{\mathscr{H}^{>}\left(\mathscr{E}_{s(\gamma)}\right)}$ for all $\chi^{>} \in \mathcal{C}_{c}\left(\mathscr{E}_{s(\gamma)}\right)$ and all $\gamma \in \mathscr{E}$.

Note that all the examples of 4.5 satisfy (H3) and (H4).

The following result is proved in [Par07], 7.3.12, the proof involves some additional technical constructions which we prefer to omit here. 
Definition and Proposition 4.8. Let $E$ be a $\mathscr{G}$-Banach $B$-pair. Define $\alpha_{\gamma}^{<}: \Gamma_{c}\left(\mathscr{g}^{s(\gamma)}, r^{*} E^{<}\right) \rightarrow \Gamma_{c}\left(\mathscr{g}^{r(\gamma)}, r^{*} E^{<}\right), \quad \xi^{<} \mapsto \gamma \xi^{<}:=\left(\gamma^{\prime} \mapsto \gamma \xi^{<}\left(\gamma^{-1} \gamma^{\prime}\right)\right)$, and

$$
\alpha_{\gamma}^{>}: \Gamma_{c}\left(\mathscr{G}_{s(\gamma)}, r^{*} E^{>}\right) \rightarrow \Gamma_{c}\left(\mathscr{E}_{r(\gamma)}, r^{*} E^{>}\right), \quad \xi^{>} \mapsto \gamma \xi^{>}:=\left(\gamma^{\prime} \mapsto \xi^{>}\left(\gamma^{\prime} \gamma\right)\right),
$$

for all $\gamma \in \mathscr{G}$. Then $\alpha_{\gamma}^{<}$and $\alpha_{\gamma}^{>}$are isometric for all $\gamma \in \mathscr{E}$ and extend to isometric isomorphisms $\mathscr{H}^{<}\left(\mathscr{E}^{s(\gamma)}, r^{*} E^{<}\right) \rightarrow \mathscr{H}^{<}\left(\mathscr{E}^{r(\gamma)}, r^{*} E^{<}\right)$and $\mathscr{H}^{>}\left(\mathscr{E}_{s(\gamma)}, r^{*} E^{>}\right) \rightarrow$ $\mathscr{H}^{>}\left(\mathscr{G}_{r(\gamma)}, r^{*} E^{>}\right)$, respectively. The field $\left(\alpha_{\gamma}^{<}, \alpha_{\gamma}^{>}\right)_{\gamma \in \mathcal{E}}$ is a continuous field of isomorphisms making $\mathfrak{F}(\mathscr{H}(\mathcal{G}, E))$ a $\mathcal{E}$-Banach $B$-pair.

Now that we assume that $\mathscr{H}(\mathscr{E})$ does not only satisfy (H1) and (H2) but also (H3) and (H4), we can refine Proposition 4.6 as follows:

Proposition 4.9. Let $E$ be a $\mathscr{G}$-Banach $A$-B-pair. Then $\mathfrak{F}(\mathscr{H}(\mathcal{E}, E))$ is a $\mathscr{E}$-Banach $B$-pair on which $\mathcal{A}(\mathscr{G}, A)$ acts by bounded $\mathscr{E}$-equivariant fields of linear operators. If $\mathcal{G}$ is proper and $\Gamma(X, A)$ acts on $E$ by locally compact operators, then the action of $\mathcal{A}(\mathscr{G}, A)$ on $\mathfrak{F}(\mathscr{H}(\mathscr{G}, E))$ is by $\mathscr{E}$-equivariant bounded locally compact fields of operators.

Because $B$ is non-degenerate, $\Gamma(X, B)$ acts on $B$ by locally compact operators. Hence we have:

Corollary 4.10. If $\mathscr{E}$ is proper, then $\mathcal{A}(\mathcal{E}, B)$ acts on $\mathfrak{F} \mathscr{H}(\mathscr{E}, B)$ by locally compact $\mathcal{G}$-equivariant operators.

To finish this section, we state and prove an extension result which shows that the bracket on $\mathscr{H}(\mathscr{E}, E)$ is the restriction of the convolution product. This fact will be used in Section 4.5.3 and also in Section A.3, in the case $E=B$.

Lemma 4.11. Let $E$ be a $\mathscr{E}$-Banach B-pair. Then the convolution

$$
\begin{gathered}
\Gamma_{c}\left(\mathcal{G}, r^{*} E^{<}\right) \times \Gamma_{c}\left(\mathcal{E}, r^{*} E^{>}\right) \rightarrow \Gamma_{c}\left(\mathscr{G}, r^{*} B\right), \\
\left(\xi^{<}, \xi^{>}\right) \mapsto \xi^{<} * \xi^{>}=\left(\gamma \mapsto \int_{\mathscr{g} r(\gamma)}\left\langle\xi^{<}\left(\gamma^{\prime}\right), \gamma^{\prime} \xi^{>}\left(\gamma^{\prime-1} \gamma\right)\right\rangle_{E_{r(\gamma)}} \mathrm{d} \lambda^{r(\gamma)}\left(\gamma^{\prime}\right)\right)
\end{gathered}
$$

extends to a contractive bilinear map

$$
\mathscr{H}^{<}\left(\mathscr{E}, E^{<}\right) \times \mathscr{H}^{>}\left(\mathscr{E}, E^{>}\right) \rightarrow \Gamma_{0}\left(\mathscr{E}, r^{*} B\right),
$$

(also) written as a convolution product, such that the bracket on $\mathscr{H}(\mathcal{G}, E)$ is the composition of this map and the restriction map from $\Gamma_{0}\left(\mathcal{G}, r^{*} B\right)$ to $\Gamma_{0}(X, B)$. 
Proof. Let $\xi^{<} \in \Gamma_{c}\left(\mathscr{G}, r^{*} E^{<}\right)$and $\xi^{>} \in \Gamma_{c}\left(\mathscr{G}, r^{*} E^{>}\right)$. For all $\gamma \in \mathscr{H}$, we have

$$
\left(\xi^{<} * \xi^{>}\right)(\gamma)=\left\langle\xi_{r(\gamma)}^{<}, \gamma \xi_{s(\gamma)}^{>}\right\rangle_{r(\gamma)}
$$

and hence

$$
\begin{aligned}
& \left\|\left(\xi^{<} * \xi^{>}\right)(\gamma)\right\|=\left\|\left\langle\xi_{r(\gamma)}^{<}, \gamma \xi_{s(\gamma)}^{>}\right\rangle_{r(\gamma)}\right\| \\
& \leq\left\|\xi_{r(\gamma)}^{<}\right\|\left\|_{\mathscr{H}<\left(\mathscr{g}, E^{<}\right)_{r(\gamma)}}\right\| \gamma \xi_{s(\gamma)}^{>} \|_{\mathscr{H}^{>}\left(\mathscr{G}, E^{>}\right)_{r(\gamma)}} \\
& =\left\|\xi_{r(\gamma)}^{<}\right\|_{\mathscr{H}^{<}\left(\mathscr{g}, E^{<}\right)_{r(\gamma)}}\left\|\xi_{s(\gamma)}^{>}\right\|_{\mathscr{H}^{>}\left(\mathscr{E}, E^{>}\right)_{s(\gamma)}} \\
& \leq\left\|\xi^{<}\right\|_{\mathscr{H}<\left(\mathscr{E}, E^{<}\right)}\left\|\xi^{>}\right\|_{\mathscr{H}>\left(\mathscr{E}, E^{>}\right)}
\end{aligned}
$$

because $\mathscr{H}^{>}(\mathscr{G})$ satisfies (H4). Hence the convolution is continuous with norm $\leq 1$ and extends to a map $\mathscr{H}^{<}\left(\mathscr{G}, E^{<}\right) \times \mathscr{H}^{>}\left(\mathscr{E}, E^{>}\right) \rightarrow \Gamma_{0}\left(\mathscr{G}, r^{*} B\right)$ with the desired properties.

4.3. Regular unconditional completions. For simplicity, we introduce the following abbreviation:

Definition 4.12. An unconditional completion $\mathcal{A}(\mathscr{G})$ of $\mathcal{\ell}_{c}(\mathscr{G})$ is said to be regular if there exists a pair $\mathscr{H}(\mathscr{E})$ of monotone completions of $\mathcal{C}_{c}(\mathscr{G})$ which satisfies (H1)-(H4).

Note that there might exist many different such pairs of monotone completions on which a regular unconditional completion acts, the important part of the definition really is the existence of such a pair, not its particular shape.

Regularity is essential in our construction of the homomorphism $M_{\mathcal{A}}^{B}$ down below.

Examples 4.13. Most examples of unconditional completions that we have come across so far are regular for rather obvious reasons:

(1) The unconditional completion $\mathrm{L}^{1}(\mathscr{G})$ acts on the pair $\left(\mathrm{L}^{1}(\mathscr{E}), \mathcal{C}_{0}(\mathscr{G})\right)$.

(2) The symmetrised version $\mathrm{L}^{1}(\mathscr{E}) \cap \mathrm{L}^{1}(\mathscr{E})^{*}$ is also regular because the norm defining it dominates the norm $\|\cdot\|_{1}$. Further, it acts on the pair $\left(\mathrm{L}_{r}^{2}(\mathscr{E}), \mathrm{L}_{s}^{2}(\mathscr{E})\right)$ (see [Ren80]). It should not be too hard to check that it also acts on $\left(\mathrm{L}_{r}^{p^{\prime}}(\mathscr{E}), \mathrm{L}_{s}^{p}(\mathscr{E})\right)$ for all $\left.p, p^{\prime} \in\right] 1, \infty\left[\right.$ such that $\frac{1}{p}+\frac{1}{p^{\prime}}=1$.

(3) The completion $\mathcal{A}_{\max }(\mathscr{G})$ acts on $\left(\mathrm{L}_{r}^{2}(\mathscr{E}), \mathrm{L}_{s}^{2}(\mathscr{E})\right)$ by definition; see Section 3 of [Laf06].

(4) If $G$ is a locally compact Hausdorff group acting on some locally compact Hausdorff space $X$, then $\mathrm{L}^{1}\left(G, \mathcal{C}_{0}(X)\right)$ is a regular completion of $\mathcal{C}_{c}(G \ltimes X)$ because its norm dominates the norm of the regular completion $\mathrm{L}^{1}(G \ltimes X)$.

There are cases of non-regular unconditional norms even when $G$ is a group, see Example A.6. 
4.4. The (inverse) homomorphism $\boldsymbol{M}_{\mathcal{A}}^{\boldsymbol{B}}$. In Section 4.4 , let $\mathcal{E}$ be proper and $\mathcal{A}(\mathcal{E})$ be regular.

Recall that we used the name $\pi$ for the canonical projection from $X$ to $X / \mathscr{E}$. Let $\pi$ also denote the map from $\mathscr{E}$ to $X / \mathscr{E}$ that maps $\gamma$ to $\pi(r(\gamma))=\pi(s(\gamma))$ (which extends $\pi: X \rightarrow X / \mathscr{E}$ ). If we regard $X / \mathscr{E}$ as a locally compact Hausdorff groupoid, then the map $\pi: \mathscr{E} \rightarrow X / \mathscr{E}$ is actually a strict morphism of groupoids. If $E$ is a u.s.c. field of Banach spaces over $X / \mathscr{G}$, then $\pi^{*} E$ is a $\mathscr{G}$-Banach space (with a rather trivial action). ${ }^{6}$ If $T$ is a continuous field of linear maps between u.s.c. fields of Banach spaces over $X / \mathcal{E}$, then $\pi^{*} T$ is an $\mathcal{E}$-equivariant continuous field of linear maps between $\mathscr{G}$-Banach spaces. We use these facts to define our "inverse homomorphism":

(1) The first step is the $\operatorname{map}^{7} \mathfrak{F}(\cdot)$ which yields a homomorphism $\mathfrak{F}(\cdot)$ :

$$
\mathcal{R K K}^{\text {ban }}\left(\mathcal{C}_{0}(X / \mathscr{E}) ; \mathcal{C}_{0}(X / \mathscr{E}), \mathcal{A}(\mathscr{E}, B)\right) \rightarrow \mathrm{KK}_{X / \mathscr{E}}^{\mathrm{ban}}\left(\mathbb{C}_{X / \mathscr{E}}, \mathfrak{F}(\mathcal{A}(\mathcal{E}, B))\right) .
$$

(2) The second step is the pullback homomorphism along $\pi$ :

$$
\pi^{*}: \mathrm{KK}_{X / \mathcal{E}}^{\mathrm{ban}}\left(\mathbb{C}_{X / \mathcal{E}}, \mathfrak{F}(\mathcal{A}(\mathcal{G}, B))\right) \rightarrow \mathrm{KK}_{\mathscr{g}}^{\mathrm{ban}}\left(\mathbb{C}_{X}, \pi^{*} \mathfrak{F}(\mathcal{A}(\mathcal{E}, B))\right) .
$$

Note that this homomorphism, on the level of cycles, produces cycles with $\mathscr{E}$ equivariant operator.

(3) Pick a pair $\mathscr{H}(\mathscr{E})$ of monotone completions of $\mathcal{C}_{c}(\mathscr{G})$ satisfying $(\mathrm{H} 1)-(\mathrm{H} 4)$. Note that there is a canonical action of $\pi^{*} \mathfrak{F}(\mathcal{A}(\mathscr{G}, B))$ on $\mathfrak{F}(\mathscr{H}(\mathscr{G}, B))$.

By Corollary 4.7, the algebra $\mathcal{A}(\mathcal{G}, B)$ acts on $\mathfrak{F}(\mathscr{H}(\mathcal{G}, B))$ by locally compact operators. If $\chi \in \mathscr{\ell}_{c}(X)$ and $a \in \mathcal{A}(\mathscr{E}, B)$, then $x \mapsto \chi(x) a_{\pi(x)}$ is a section of $\pi^{*}(\mathfrak{F}(\mathcal{A}(\mathscr{E}, B)))$ with compact support. Such sections act on $\mathfrak{F}(\mathcal{H}(\mathscr{E}, B))$ by locally compact operators with compact support, so they act by compact operators. By a density argument, all sections of $\pi^{*}(\mathfrak{F}(\mathcal{A}(\mathscr{E}, B)))$ that vanish at infinity act on $\mathfrak{F}(\mathscr{H}(\mathscr{E}, B))$ by compact operators. Hence we can regard $\mathfrak{F}(\mathcal{H}(\mathscr{E}, B))$ as a Morita cycle ${ }^{8}$ from $\pi^{*} \mathfrak{F}(\mathcal{A}(\mathscr{E}, B))$ to $B \cong \mathfrak{F}\left(\Gamma_{0}(X, B)\right)$. The important point is that this Morita cycle carries an action of $\mathscr{E}$ which makes it a $\mathscr{G}$-equivariant Morita cycle. Morita cycles act on $\mathrm{KK}^{\text {ban }}$ from the right, so we get a homomorphism

$$
\otimes_{\pi^{*} \mathfrak{F}(\mathcal{A}(\mathscr{E}, B))} \mathfrak{F}(\mathscr{H}(\mathscr{E}, B)): \mathrm{KK}_{\mathscr{g}}^{\mathrm{ban}}\left(\mathbb{C}_{X}, \pi^{*} \mathfrak{F}(\mathcal{A}(\mathscr{E}, B))\right) \rightarrow \mathrm{KK}_{\mathscr{g}}^{\mathrm{ban}}\left(\mathbb{C}_{X}, B\right) .
$$

If a cycle has a $\mathscr{G}$-equivariant operator, then it stays equivariant under this homomorphism.

The composition of these three homomorphisms gives the desired natural homomorphism,

$$
M_{\mathcal{A}, \mathscr{H}}^{B}: \mathcal{R} \mathrm{KK}^{\mathrm{ban}}\left(\mathcal{C}_{0}(X / \mathscr{E}) ; \digamma_{0}(X / \mathscr{E}), \mathcal{A}(\mathscr{E}, B)\right) \rightarrow \mathrm{KK}_{\mathscr{E}}^{\mathrm{ban}}\left(\mathbb{C}_{X}, B\right),
$$

\footnotetext{
${ }^{6}$ See [Laf06] or [Par09a], Section 4.1, for a definition of the pullback along strict morphisms.

${ }^{7}$ See Section 1.3 or [Par07], Chapter 4, for a definition of the functor $\mathfrak{F}(\cdot)$.

${ }^{8}$ See [Par09a], Section 3.3.6, for a definition; compare [Par09b], Definition 5.7.
} 
which produces cycles with $\mathcal{E}$-equivariant operators.

Proposition 4.14. Let $\mathscr{H}^{\prime}(\mathscr{G})=\left(\mathscr{H}^{\prime<}(\mathscr{E}), \mathscr{H}^{\prime>}(\mathscr{E})\right)$ be another pair of monotone completions of $\mathcal{C}_{c}(\mathscr{E})$ satisfying $(\mathrm{H} 1)-(\mathrm{H} 4)$. Then the natural homomorphisms $M_{\mathcal{A}, \mathcal{H}}^{B}$ and $M_{\mathscr{A}, \mathscr{H}^{\prime}}^{B}$ are equal. We call this natural homomorphism $M_{\mathcal{A}}^{B}$.

Proof. We first consider the case that $\|\cdot\| \mathscr{H}^{<} \leq\|\cdot\|_{\mathscr{H}^{\prime}}$ and $\|\cdot\| \mathscr{H}>\leq\|\cdot\|_{\mathscr{H}^{\prime}}$. In this case, we have a canonical homomorphism $\Phi$ from $\mathscr{H}^{\prime}(\mathcal{E}, B)$ to $\mathscr{H}(\mathcal{E}, B)$ which gives us an equivariant homomorphism $\mathfrak{F}(\Phi)$ from $\mathfrak{F}\left(\mathscr{H}^{\prime}(\mathcal{E}, B)\right)$ to $\mathfrak{F}(\mathscr{H}(\mathscr{E}, B))$. The homomorphism $\mathfrak{F}(\Phi)$ is actually a morphism of equivariant Morita cycles from $\pi^{*} \mathfrak{F}(\mathcal{A}(\mathcal{E}, B))$ to $B$. A careful revision of the proof that $\pi^{*} \mathfrak{F}(\mathcal{A}(\mathcal{E}, B))$ acts by compact operators on $\mathfrak{F}\left(\mathscr{H}^{\prime}(\mathcal{E}, B)\right)$ and on $\mathfrak{F}(\mathscr{H}(\mathscr{E}, B))$ shows that $\mathfrak{F}(\Phi)$ satisfies the conditions of Theorem 3.20 of [Par09a] and hence induces a homotopy from $\mathfrak{F}\left(\mathscr{H}^{\prime}(\mathscr{G}, B)\right)$ to $\mathfrak{F}(\mathscr{H}(\mathscr{E}, B))$. So $M_{\mathscr{A}, \mathscr{H}^{\prime}}^{B}=M_{\mathscr{A}, \mathscr{H}}^{B}$ because the tensor product with Morita cycles lifts to homotopy classes. ${ }^{9}$

Now consider the general case. By taking the maximum of the norms on $\mathscr{H}^{<}(\mathscr{G})$ and $\mathscr{H}^{\prime<}(\mathscr{G})$ we define a monotone completion $\mathscr{H}^{\prime \prime<}(\mathscr{G})$ of $\mathscr{C}_{c}(\mathscr{G})$; similarly, we define $\mathscr{H}^{\prime \prime>}(\mathscr{G})$. The pair $\mathscr{H}^{\prime \prime}(\mathscr{E}):=\left(\mathscr{H}^{\prime \prime<}(\mathscr{E}), \mathscr{H}^{\prime \prime>}(\mathscr{E})\right)$ also satisfies $(\mathrm{H} 1)-(\mathrm{H} 4)$. By the first part of the proof we can conclude $M_{\mathcal{A}, \mathscr{H}}^{B}=M_{\mathscr{A}, \mathscr{H}^{\prime \prime}}^{B}=M_{\mathcal{A}, \mathscr{H}^{\prime}}^{B}$.

4.5. $\boldsymbol{J}_{\mathcal{A}}^{\boldsymbol{B}} \circ \boldsymbol{M}_{\mathscr{A}}^{\boldsymbol{B}}=\mathbf{I d}$ on the level of $\mathbf{K K}^{\text {ban }}$. We now prove Theorem 2.3. Let $\mathcal{E}$ be proper and let $\mathcal{A}(\mathcal{G})$ be regular. Assume moreover that $\mathcal{E}$ admits a cut-off function.

4.5.1. Idea of the proof. First, we choose a pair $\mathcal{D}(X)$ of monotone completions of $\mathcal{C}_{c}(X)$ satisfying (D1) and (D2). Second, because $\mathcal{A}(\mathscr{\mathscr { S }})$ is regular, we can also choose a pair $\mathscr{H}(\mathscr{H})=\left(\mathscr{H}^{<}(\mathscr{G}), \mathscr{H}^{>}(\mathscr{G})\right)$ of monotone completions of $\boldsymbol{C}_{c}(\mathscr{E})$ satisfying (H1)-(H4). Let $(E, T) \in \mathbb{E}^{\text {ban }}\left(\mathcal{C}_{0}(X / \mathscr{G}) ; \mathcal{C}_{0}(X / \mathcal{E}), \mathcal{A}(\mathscr{E}, B)\right)$. We have to show that $(E, T)$ is homotopic to $J_{\mathscr{A}, \mathscr{D}}^{B}\left(M_{\mathcal{A}, \mathscr{H}}^{B}(E, T)\right)$. The obvious strategy is to define a morphism from $J_{\mathscr{A}, \mathscr{D}}^{B}\left(M_{\mathscr{A}, \mathscr{H}}^{B}(E)\right)$ to $E$ which induces a homotopy; there is a canonical candidate for such a morphism defined on a dense subspace, but this candidate does not extend to a continuous morphism on the entire space: The norms on $J_{\mathscr{A}, \mathscr{D}}^{B}\left(M_{\mathscr{A}, \mathscr{H}}^{B}(E)\right)$ and $E$ seem to be difficult to compare in general.

We overcome this problem by constructing a pair $\widetilde{E}:=\left(\widetilde{E}^{<}, \widetilde{E}^{>}\right)$of $\mathbb{C}$-vector spaces which are equipped with compatible $\mathcal{C}_{c}(X / \mathscr{E})$-module structures and left/right $\Gamma_{c}\left(\mathcal{G}, r^{*} B\right)$-module structures and a bilinear map from $\widetilde{E}^{<} \times \widetilde{E}^{>}$to $\Gamma_{c}\left(\mathcal{G}, r^{*} B\right)$. On this pair, which could be called a "pre-A( $\mathcal{G}, B)$-pair", we construct a pair of formally adjoint operators $\widetilde{T}$. Moreover, we define canonical "homomorphisms" $\Phi_{E}$ from $\widetilde{E}$ to $E$ and $\Psi_{E}$ from $\widetilde{E}$ to $J_{\mathscr{A}, \mathscr{D}}^{B}\left(M_{\mathcal{A}, \mathscr{H}}^{B}(E)\right)$ which intertwine $\widetilde{T}$ and $T$ and

\footnotetext{
${ }^{9}$ See [Par09a], Section 3.3.6, or [Par07], Section 3.8.
} 
$J_{\mathscr{A}, \mathscr{D}}^{B}\left(M_{\mathscr{A}, \mathscr{H}}^{B}(T)\right)$, respectively:

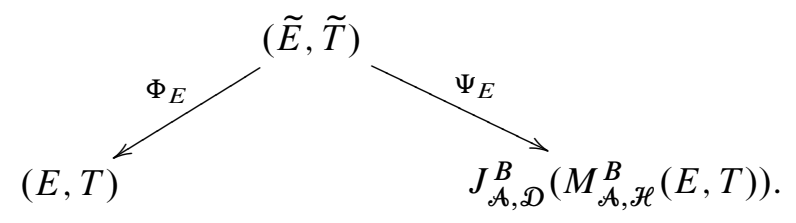

One can think of $\widetilde{E}$ as a dense subspace of both, $E$ and $J_{\mathcal{A}, \mathscr{D}}^{B}\left(M_{\mathcal{A}, \mathscr{H}}^{B}(E)\right)$. Now we put on $\widetilde{E}$ the supremum of the semi-norms which are induced by the two homomorphisms, making the homomorphisms continuous. We then show that the completion of $\widetilde{E}$ together with the continuous extension of $\widetilde{T}$ is in $\mathbb{E}^{\mathrm{ban}}\left(\mathcal{C}_{0}(X / \mathscr{E}) ; \mathcal{C}_{0}(X / \mathscr{G}), \mathcal{A}(\mathscr{E}, B)\right)$ and that the two homomorphisms induce homotopies. Hence also $(E, T)$ and $J_{\mathscr{A}, \mathcal{D}}^{B}\left(M_{\mathscr{A}, \mathscr{H}}^{B}(E, T)\right)$ are homotopic.

4.5.2. A sufficient condition for homotopy. The central tool to construct these homotopies is the following sufficient condition for the homotopy of $\mathcal{R} \mathrm{KK}^{\mathrm{ban}}$-cycles that generalises a corresponding theorem for $\mathrm{KK}^{\text {ban }}$-cycles, compare [Par09b], Theorem 2.1. The main idea is that the mapping cylinder of a homomorphism $\Phi$ of $\mathcal{R} \mathrm{KK}^{\text {ban }}$-cycles gives a homotopy between the cycles. For this to be true, $\Phi$ has to satisfy a technical condition which says that the operators which are required to be compact in the definition of $\mathcal{R} \mathrm{KK}^{\mathrm{ban}}$-cycles can be approximated simultaneously by finite rank operators for both cycles which $\Phi$ connects. This is what is meant by “ $\left(\Phi,\left(T, T^{\prime}\right)\right) \in \mathbb{E}^{\mathrm{ban}}\left(\mathcal{C}_{0}(Z) ; \operatorname{Id}_{A}, \operatorname{Id}_{B}\right)$ ” in the following theorem:

Theorem 4.15. Let $Z$ be a locally compact Hausdorff space and let $C$ and $D$ be $\mathcal{C}_{0}(Z)$-Banach algebras. Let $(E, T),\left(E^{\prime}, T^{\prime}\right)$ be elements of $\mathbb{E}^{\text {ban }}\left(\mathcal{C}_{0}(Z) ; C, D\right)$. If there is a $\ell_{0}(Z)$-linear morphism $\Phi$ from $(E, T)$ to $\left(E^{\prime}, T^{\prime}\right)$ (with coefficient maps $\operatorname{Id}_{C}$ and $\left.\operatorname{Id}_{D}\right)$ such that $\left(\Phi,\left(T, T^{\prime}\right)\right) \in \mathbb{E}^{\text {ban }}\left(\mathcal{C}_{0}(Z) ; \operatorname{Id}_{C}, \operatorname{Id}_{D}\right)$, then $(E, T) \sim\left(E^{\prime}, T^{\prime}\right)$.

The necessary concepts are explained in [Par09b] for $\mathrm{KK}^{\mathrm{ban}}$; to obtain the result for $\ell_{0}(Z)$-Banach algebras it suffices to add compatible $\ell_{0}(Z)$-Banach spaces structures. You can also prove an equivariant version by simply adding group actions everywhere.

4.5.3. The construction of $\widetilde{E}, \Phi_{E}$ and $\Psi_{E}$. We are going to cut the proof into a series of statements and definitions. In this section, let $E$ and $F$ be $\mathcal{C}_{0}(X / \mathcal{E})$-Banach $\mathcal{A}(\mathscr{E}, B)$-pairs.

The pair $\widetilde{E}$ : Define

$$
\widetilde{E}^{>}:=E^{>} \otimes_{\Gamma_{c}\left(\mathcal{E}, r^{*} B\right)} \Gamma_{c}\left(\mathcal{E}, r^{*} B\right)
$$


and

$$
\widetilde{E}^{<}:=\Gamma_{c}\left(\mathscr{G}, r^{*} B\right) \otimes_{\Gamma_{c}\left(\mathscr{G}, r^{*} B\right)} E^{<}
$$

These vector spaces carry canonical and compatible actions of $\Gamma_{c}\left(\mathcal{G}, r^{*} B\right)$ and $\ell_{c}(X / \mathcal{G})$. A bracket on $\widetilde{E}$ is defined by

$$
\begin{gathered}
\langle\cdot, \cdot\rangle: \widetilde{E}^{<} \times \widetilde{E}^{>} \rightarrow \Gamma_{c}\left(\mathscr{E}, r^{*} B\right), \\
\left\langle\beta^{<} \otimes e^{<}, e^{>} \otimes \beta^{>}\right\rangle(\gamma):=\beta^{<} *\left\langle e^{<}, e^{>}\right\rangle * \beta^{>}=\left\langle\beta^{<} e^{<}, e^{>} \beta^{>}\right\rangle .
\end{gathered}
$$

We check that the bracket has indeed its values in $\Gamma_{c}\left(\mathcal{G}, r^{*} B\right)$ : The element $\left\langle e^{<}, e^{>}\right\rangle$is in $\mathcal{A}(\mathcal{E}, B)$ by definition, and we now show that the product $\beta^{<} * \beta * \beta^{>}$ is in $\Gamma_{c}(\mathscr{H}, B)$ for all $\beta^{<}, \beta^{>} \in \Gamma_{c}(\mathscr{H}, B)$ and $\beta \in \mathcal{A}(\mathcal{H}, B)$. If we regard $\beta^{<}$as an element of $\mathscr{H}^{<}(\mathcal{H}, B)$ and $\beta^{>}$as an element of $\mathscr{H}^{>}(\mathcal{E}, B)$, then we can conclude from Lemma 4.11 that the map $\beta \mapsto \beta^{<*} \beta * \beta^{>}$is continuous from $\mathcal{A}(\mathscr{H}, B)$ to $\Gamma_{0}(\mathscr{E}, B)$ because $\mathcal{A}(\mathscr{G})$ acts on $\mathscr{H}(\mathscr{G})$. Moreover, the support of the product $\beta^{<} * \beta * \beta^{>}$is always contained in the set $\left\{\gamma \in \mathcal{G}: r(\gamma) \in r\left(\operatorname{supp} \beta^{<}\right), s(\gamma) \in s\left(\operatorname{supp} \beta^{>}\right)\right\}$, which is compact because $\mathcal{E}$ is proper.

The map $\Phi_{E}$ : Define

$$
\Phi_{E}^{>}: \widetilde{E}^{>} \rightarrow E^{>}, \quad e^{>} \otimes \beta^{>} \mapsto e^{>} \beta^{>},
$$

and

$$
\Phi_{E}^{<}: \widetilde{E}^{<} \rightarrow E^{<}, \quad \beta^{<} \otimes e^{<} \mapsto \beta^{<} e^{<} .
$$

Both maps are obviously $\Gamma_{c}\left(\mathcal{G}, r^{*} B\right)$ - and $\mathcal{C}_{c}(X / \mathcal{E})$-linear. Furthermore, the pair $\Phi_{E}=\left(\Phi_{E}^{<}, \Phi_{E}^{>}\right)$is compatible with the brackets on $\widetilde{E}$ and $E$.

The map $\Psi_{E}$ : Let $e^{>} \in E^{>}$and $\beta^{>} \in \Gamma_{c}\left(\mathcal{E}, r^{*} B\right)$. Since $\beta^{>}$has compact support, the function $x \mapsto\left(e^{>} \otimes \beta^{>}\right)_{x}=e_{\pi(x)}^{>} \otimes \beta_{x}^{>}$is in $\Gamma_{c}\left(X, \pi^{*} \mathfrak{F}\left(E^{>}\right) \otimes_{\pi^{*} \mathfrak{F}(\mathcal{A}(\mathscr{E}, B))}\right.$ $\left.\mathfrak{F}\left(\mathscr{H}^{>}(\mathcal{E}, B)\right)\right)$; we can regard this function as an element $\Psi_{E}^{>}\left(e^{>} \otimes \beta^{>}\right)$of $\mathscr{D}^{>}\left(X, \pi^{*} \mathfrak{F}\left(E^{>}\right) \otimes_{\pi^{*} \mathfrak{F}(\mathcal{A}(\mathscr{E}, B))} \mathfrak{F}\left(\mathscr{H}^{>}(\mathcal{E}, B)\right)\right)$; here $\pi: X \rightarrow X / \mathcal{G}$ denotes the canonical projection. This gives rise to a map $\Psi_{E}^{>}$from $\widetilde{E}^{>}$to $J_{\mathscr{A}, \mathscr{D}}^{B}\left(M_{\mathscr{A}, \mathscr{H}}^{B}(E)\right)^{>}$.

Similarly we define

$$
\Psi_{E}^{<}\left(\beta^{<} \otimes e^{<}\right)_{x}:=\beta_{x}^{<} \otimes e_{\pi(x)}^{<} \in \mathscr{H}^{<}(\mathcal{E}, B)_{x} \otimes_{\mathcal{A}(\mathcal{E}, B)_{\pi(x)}} E_{\pi(x)}^{<}
$$

for all $e^{<} \in E^{<}, \beta^{<} \in \Gamma_{c}\left(\mathcal{G}, r^{*} B\right)$ and $x \in X$, giving us a $\Gamma_{c}\left(\mathcal{E}, r^{*} B\right)$-linear and $\ell_{c}(X / \mathscr{G})$-linear map $\Psi_{E}^{<}$from $\tilde{E}^{<}$to $J_{\mathscr{A}, \mathcal{D}}^{B}\left(M_{\mathcal{A}, \mathcal{H}}^{B}(E)\right)^{<}$. The pair $\Psi_{E}=\left(\Psi_{E}^{<}, \Psi_{E}^{>}\right)$ is compatible with the brackets on $\widetilde{E}$ and $J_{\mathscr{A}, \mathscr{D}}^{B}\left(M_{\mathcal{A}, \mathscr{H}}^{B}(E)\right)$.

The constructions for linear operators: Let $S \in \mathrm{L}_{\mathcal{A}(\mathscr{E}, B)}(E, F)$ be an operator between the $\mathcal{C}_{0}(X / \mathcal{G})$-Banach $\mathcal{A}(\mathcal{G}, B)$-pairs $E$ and $F$. Define

$$
\tilde{S}^{>}: \tilde{E}^{>} \rightarrow \tilde{F}^{>}, \quad \xi^{>} \otimes \beta^{>} \mapsto S^{>}\left(\xi^{>}\right) \otimes \beta^{>},
$$

and

$$
\tilde{S}^{<}: \tilde{F}^{<} \rightarrow \tilde{E}^{<}, \quad \beta^{<} \otimes \xi^{<} \mapsto \beta^{<} \otimes S^{<}\left(\xi^{<}\right) .
$$


Note that $\widetilde{S}:=\left(\widetilde{S}^{<}, \widetilde{S}^{>}\right)$is formally adjoint in the following sense:

$$
\begin{aligned}
\left\langle\widetilde{S}^{<}\left(\beta^{<} \otimes \xi^{<}\right), \xi^{>} \otimes \beta^{>}\right\rangle & =\beta^{<} *\left\langle S^{<}\left(\xi^{<}\right), \xi^{>}\right\rangle * \beta^{>} \\
& =\beta^{<} *\left\langle\xi^{<}, S^{>}\left(\xi^{>}\right)\right\rangle * \beta^{>} \\
& =\left\langle\beta^{<} \otimes \xi^{<}, \tilde{S}^{>}\left(\xi^{>} \otimes \beta^{>}\right)\right\rangle
\end{aligned}
$$

for all $\beta^{<}, \beta^{>} \in \Gamma_{c}\left(\mathscr{G}, r^{*} B\right), \xi^{<} \in \Gamma_{c}\left(X, F^{<}\right)$and $\xi^{>} \in \Gamma_{c}\left(X, E^{>}\right)$.

By direct calculation one checks:

(1) The maps $\Phi_{E}$ and $\Phi_{F}$ intertwine $\widetilde{S}$ and $S$ in the obvious sense.

(2) The maps $\Psi_{E}$ and $\Psi_{F}$ intertwine $\widetilde{S}$ and $J_{\mathscr{A}, \mathscr{D}}^{B}\left(M_{\mathcal{A}, \mathscr{H}}^{B}(S)\right)$.

4.5.4. Putting a norm on $\widetilde{\boldsymbol{E}}$. If $\tilde{e}^{>} \in \widetilde{E}^{>}$, then define

$$
\left\|\tilde{e}^{>}\right\|:=\max \left\{\left\|\Phi_{E}^{>}\left(\tilde{e}^{>}\right)\right\|,\left\|\Psi_{E}^{>}\left(\tilde{e}^{>}\right)\right\|\right\} .
$$

This is a semi-norm on $\widetilde{E}^{>}$. Let $\bar{E}^{>}$be the (Hausdorff-) completion of $\widetilde{E}^{>}$with respect to this semi-norm. In an analogous fashion, define a semi-norm on $\widetilde{E}^{<}$and call the completion $\bar{E}^{<}$. The actions of $\Gamma_{c}\left(\mathscr{E}, r^{*} B\right)$ and $\mathcal{C}_{c}(X / \mathscr{E})$ on $\widetilde{E}$ extend to non-degenerate actions of $\mathcal{A}(\mathscr{E}, B)$ and $\mathcal{C}_{0}(X / \mathscr{E})$ on $\bar{E}$. The bracket on $\widetilde{E}$ extends to a continuous bracket on $\bar{E}$, making $\bar{E}$ a $\mathcal{C}_{0}(X / \mathcal{E})$-Banach $\mathcal{A}(\mathcal{E}, B)$-pair.

Now the map $\Phi_{E}^{>}$extends by continuity to a continuous linear map from $\bar{E}^{>}$to $E$ which is $\mathcal{A}(\mathscr{G}, B)$ - and $\mathcal{C}_{0}(X / \mathscr{G})$-linear. Similar things can be said about $\Phi_{E}^{<}, \Psi_{E}^{>}$and $\Psi_{E}^{<}$. We get homomorphisms $\Phi_{E}$ from $\bar{E}$ to $E$ and $\Psi_{E}$ from $\bar{E}$ to $J_{\mathscr{A}, \mathcal{D}}^{B}\left(M_{\mathcal{A}, \mathscr{H}}^{B}(E)\right)$.

Let $S \in \mathrm{L}_{\mathcal{A}(\mathscr{E}, B)}(E, F)$ as above. Then the map $\tilde{S}^{>}$satisfies

$$
\left\|\tilde{S}^{>}\left(\tilde{e}^{>}\right)\right\| \leq\left\|S^{>}\right\|\left\|\tilde{e}^{>}\right\|
$$

for all $\tilde{e}^{>} \in \widetilde{E}^{>}$and extends therefore to an operator $\bar{S}^{>}$from $\bar{E}^{>}$to $\bar{F}^{>}$. Analogously for $\tilde{S}^{<}$. We thus get an element $\bar{S} \in \mathrm{L}_{\mathcal{A}(\mathscr{S}, B)}(\bar{E}, \bar{F})$ of norm $\leq\|S\|$. The map $S \mapsto \dot{\bar{S}}$ is $\mathbb{C}$-linear and functorial. The homomorphisms $\Phi_{E}$ and $\Phi_{F}$ intertwine $\bar{S}$ and $S$ in the obvious sense and the homomorphisms $\Psi_{E}$ and $\Psi_{F}$ intertwine $\bar{S}$ and $J_{\mathscr{A}, \mathscr{D}}^{B}\left(M_{\mathcal{A}, \mathscr{H}}^{B}(S)\right)$.

By direct comparison of the operators one can show:

Lemma 4.16. Let $e^{<} \in \Gamma_{0}\left(X, E^{<}\right), f^{>} \in \Gamma_{0}\left(X, F^{>}\right), \beta^{<}, \beta^{>} \in \Gamma_{c}\left(\mathcal{G}, r^{*} B\right)$. If

$$
S=\left|f^{>} \beta^{>}\right\rangle\left\langle\beta^{<} e^{<}\right| \in \mathrm{K}_{\mathcal{A}(\mathcal{E}, B)}(E, F),
$$

then

$$
\bar{S}=\left|f^{>} \otimes \beta^{>}\right\rangle\left\langle\beta^{<} \otimes e^{<}\right| \in \mathrm{K}_{\mathcal{A}(\mathcal{G}, B)}(\bar{E}, \bar{F})
$$

and

$$
\begin{aligned}
J_{\mathscr{A}, \mathcal{D}}^{B}\left(M_{\mathcal{A}, \mathscr{H}}^{B}(S)\right)= & \left|\Psi_{F}^{>}\left(f^{>} \otimes \beta^{>}\right)\right\rangle\left\langle\Psi_{E}^{<}\left(\beta^{<} \otimes e^{<}\right)\right| \\
& \in \mathrm{K}_{\mathcal{A}(\mathscr{E}, B)}\left(J_{\mathcal{A}, \mathscr{D}}^{B}\left(M_{\mathcal{A}, \mathscr{H}}^{B}(E)\right), J_{\mathcal{A}, \mathscr{D}}^{B}\left(M_{\mathcal{A}, \mathcal{H}}^{B}(F)\right)\right) .
\end{aligned}
$$


It follows for all $S \in \mathrm{K}_{\mathcal{A}(\mathcal{E}, B)}(E, F)$ that $\bar{S}$ and $J_{\mathcal{A}, \mathscr{D}}^{B}\left(M_{\mathcal{A}, \mathcal{H}}^{B}(S)\right)$ are compact and that $(\bar{S}, S) \in \mathrm{K}\left(\Phi_{E}, \Phi_{F}\right)$ as well as $\left(\bar{S}, J_{\mathscr{A}, \mathscr{D}}^{B}\left(M_{\mathscr{A}, \mathscr{H}}^{B}(S)\right)\right) \in \mathrm{K}\left(\Psi_{E}, \Psi_{F}\right)$. The precise definition of $\mathrm{K}\left(\Phi_{E}, \Phi_{F}\right)$ can be found in [Par09b]; compare the discussion around Theorem 4.15.

4.5.5. The proof of $\boldsymbol{J}_{\mathscr{A}}^{\boldsymbol{B}} \circ \boldsymbol{M}_{\mathscr{A}}^{\boldsymbol{B}}=\mathbf{I d}$. We show that $(\bar{E}, \bar{T})$ is homotopic to $(E, T)$ and to $J_{\mathscr{A}, \mathscr{D}}^{B}\left(M_{\mathcal{A}, \mathscr{H}}^{B}(E, T)\right)$, where $(E, T) \in \mathbb{E}^{\text {ban }}\left(\mathcal{C}_{0}(X / \mathscr{E}) ; \mathcal{C}_{0}(X / \mathscr{G}), \mathcal{A}(\mathscr{E}, B)\right)$.

If $\chi \in \mathcal{C}_{c}(X / \mathscr{E})$ and $S:=\chi\left(T^{2}-1\right)$, then $(\bar{S}, S)$ is in $\mathrm{K}\left(\Phi_{E}, \Phi_{E}\right)$ and $\left(\bar{S}, J_{\mathscr{A}, \mathscr{D}}^{B}\left(M_{\mathscr{A}, \mathscr{H}}^{B}(S)\right)\right) \in \mathrm{K}\left(\Psi_{E}, \Psi_{E}\right)$ by Lemma 4.16. If follows that $(\bar{E}, \bar{T})$ is in $\mathbb{E}^{\text {ban }}\left(\mathcal{C}_{0}(X / \mathscr{E}) ; \mathcal{C}_{0}(X / \mathscr{E}), \mathcal{A}(\mathscr{G}, B)\right)$ and, using Theorem 4.15 , that it is homotopic to $(E, T)$ as well as to $J_{\mathscr{A}, \mathscr{D}}^{B}\left(M_{\mathscr{A}, \mathscr{H}}^{B}(E, T)\right)$.

4.6. Sketch of the proof of $\boldsymbol{M}_{\mathcal{A}}^{B} \circ \boldsymbol{J}_{\mathscr{A}}^{\boldsymbol{B}}=\mathbf{I d}$. We first have to introduce an additional technical concept to be able to formulate the precise conditions under which we can show the injectivity part of the generalised Green-Julg theorem:

Let $\mathscr{H}(\mathscr{E})=\left(\mathscr{H}^{<}(\mathscr{G}), \mathscr{H}^{>}(\mathscr{E})\right)$ be a pair of monotone completions of $\mathcal{C}_{c}(\mathscr{E})$ satisfying $(\mathrm{H} 1)-(\mathrm{H} 4)$. A cut-off pair $c=\left(c^{<}, c^{>}\right)$for $\mathscr{E}$ is called an $\mathscr{H}(\mathscr{E})$-cut-off pair if

$$
\left\|\mathscr{E}_{x} \ni \gamma \mapsto c^{>}(r(\gamma))\right\|_{\mathscr{H}>\left(\mathscr{E}_{x}\right)}=1 \quad \text { and } \quad\left\|\mathscr{E}^{x} \ni \gamma \mapsto c^{<}(s(\gamma))\right\|_{\mathscr{H}<\left(\mathscr{E}^{x}\right)}=1
$$

for all $x \in X$.

Examples 4.17. Assume that $X / \mathscr{E}$ is $\sigma$-compact. Let $c$ be a cut-off-function for $\mathscr{E}$.

(1) Proposition 3.2 gives a $\mathscr{H}(\mathscr{G})$-cut-off pair $(c, d)$ for $\mathscr{H}(\mathscr{E})=\left(\mathrm{L}^{1}(\mathscr{E}), \mathscr{\ell}_{0}(\mathscr{E})\right)$.

(2) If $\left.p, p^{\prime} \in\right] 1, \infty\left[\right.$ such that $\frac{1}{p}+\frac{1}{p^{\prime}}=1$, then $\left(c^{\frac{1}{p^{\prime}}}, c^{\frac{1}{p}}\right)$ is a $\mathscr{H}(\mathscr{E})$-cut-off pair for the pair $\mathscr{H}(\mathscr{G})=\left(\mathrm{L}_{r}^{p^{\prime}}(\mathscr{G}), \mathrm{L}_{s}^{p}(\mathscr{E})\right)$.

Observe that these examples show that $\mathrm{L}^{1}(\mathscr{G}), \mathrm{L}^{1}(\mathscr{E}) \cap \mathrm{L}^{1}(\mathscr{E})^{*}$ and $\mathcal{A}_{\max }(\mathscr{E})$ are not only regular (see Examples 4.13) but satisfy the hypotheses of the generalised Green-Julg theorem 2.4 for $\sigma$-compact $X / \mathscr{E}$.

The technical reason to consider $\mathscr{H}(\mathscr{E})$-cut-off pairs is that they allow us to embed $\Gamma_{0}(X, E)$ into $\mathscr{H}(\mathscr{E}, E)$ as a direct summand, where $E$ is a $\mathscr{E}$-Banach $B$-pair. This is a Banach algebraic analogue of the fact that every $\mathscr{E}-\mathrm{C}^{*}$-algebra $B$ can be embedded into $\mathrm{L}^{2}(\mathcal{G}, B)$ as a direct summand, see Proposition 6.21 of [Tu99].

Recall that the hypotheses of our main result, Theorem 2.4, are that we are given

- an unconditional completion $\mathcal{A}(\mathscr{E})$ of $\mathcal{C}_{c}(\mathscr{\mathscr { G }})$,

- a pair $\mathscr{H}(\mathscr{G})$ of monotone completions of $\mathcal{C}_{c}(\mathscr{E})$ satisfying $(\mathrm{H} 1)-(\mathrm{H} 4)$,

- an $\mathscr{H}(\mathscr{E})$-cut-off pair $c$ for $\mathscr{E}$, and

- a non-degenerate $\mathcal{E}$-Banach algebra $B$. 
These hypotheses imply that $\mathscr{Z}$ admits a cut-off function and that $\mathcal{A}(\mathscr{G})$ is regular. Hence the surjectivity part of the theorem has already been settled; more specifically, we have $J_{\mathscr{A}}^{B} \circ M_{\mathscr{A}}^{B}=$ Id.

We want to show that, under these hypotheses, $M_{\mathcal{A}}^{B} \circ J_{\mathscr{A}}^{B}=\mathrm{Id}$ as an endomorphism of the group $\mathcal{R} \mathrm{KK}^{\mathrm{ban}}\left(\mathcal{C}_{0}(X / \mathcal{G}) ; \boldsymbol{C}_{0}(X / \mathcal{E}), \mathcal{A}(\mathcal{G}, B)\right)$.

Idea of the proof of $M_{\mathcal{A}}^{B} \circ J_{\mathcal{A}}^{B}=$ Id: Let $(E, T) \in \mathbb{E}_{\mathscr{G}}^{\text {ban }}\left(\mathcal{C}_{0}(X), B\right)$ with $\mathscr{E}$ equivariant $T$. The idea is to define a homomorphism $\Phi_{E}$ from $M_{\mathcal{A}, \mathscr{H}^{\mathcal{H}}}^{B}\left(J_{\mathscr{A}^{,} \mathcal{A}^{c}}^{B}(E)\right)$ to $E$ that commutes with the operator $M_{\mathcal{A}, \mathscr{H}}^{B}\left(J_{\mathscr{A}^{,}, \mathcal{A}^{c}}^{B}(T)\right)$ and $T$. We then show that $\Phi_{E}$ induces a homotopy by checking the technical conditions of Theorem 3.20 of [Par09a]. Note that we use the particular pair $\mathcal{A}^{c}(X)$ of monotone completions of $\ell_{c}(X)$ here, see Section 4.1.4.

The central ingredient in the construction of $\Phi_{E}$ is a homomorphism

$$
\mathcal{A}^{c}(X, E) \otimes_{\mathcal{A}(\mathscr{E}, B)} \mathscr{H}(\mathscr{G}, B) \rightarrow \Gamma_{0}(X, E) .
$$

To define it, observe that the convolution gives a homomorphism

$$
\mathcal{A}(\mathscr{E}, E) \otimes_{\mathcal{A}(\mathscr{E}, B)} \mathscr{H}(\mathscr{E}, B) \rightarrow \mathscr{H}(\mathscr{E}, E) .
$$

By definition, $\mathcal{A}^{c}(X, E)$ embeds into $\mathcal{A}(\mathscr{E}, E)$, so we can embed $\mathcal{A}^{c}(X, E) \otimes_{\mathcal{A}}(\mathscr{g}, B)$ $\mathscr{H}(\mathscr{G}, B)$ into $\mathcal{A}(\mathscr{E}, E) \otimes_{\mathcal{A}(\mathscr{E}, B)} \mathscr{H}(\mathscr{E}, B)$. On the other hand, $\Gamma_{0}(X, E)$ is contained as a direct summand in $\mathscr{H}(\mathscr{G}, E)$ because $c$ is a $\mathscr{H}(\mathscr{E})$-cut-off pair, so we can compose with the projection onto this summand to obtain the desired homomorphism. The homomorphism $\Phi_{E}$ is constructed from it by some standard operations.

The main difficulty of the proof is to check that the homomorphism $\Phi_{E}$ really gives a homotopy between $M_{\mathcal{A}_{,}, \mathcal{H}}^{B}\left(J_{\mathcal{A}, \mathcal{A}^{c}}^{B}(E, T)\right)$ and $(E, T)$. This boils down to some approximation arguments for compact operators which are carried out in detail in [Par07], Section 7.8.

\section{A. Appendix: Does $\mathrm{K}_{*}(\mathcal{A}(\mathcal{E}, B))$ depend on $\mathcal{A}(\mathscr{E})$ ?}

In this section, let $\mathcal{E}$ be a locally compact Hausdorff groupoid with Haar system and unit space $X$ and let $\mathcal{A}(\mathscr{G})$ be an unconditional completion of $\mathcal{C}_{c}(\mathscr{E})$.

\section{A.1. Properness and the invariance of K-theory}

Conjecture A.1. Let $\mathcal{E}$ be proper and let $B$ be a (possibly degenerate) $\mathcal{E}$-Banach algebra. Then the group $\mathrm{K}_{*}(\mathcal{A}(\mathscr{E}, B))$ is independent of the choice of the unconditional completion $\mathcal{A}(\mathscr{E})$ of $\mathcal{C}_{c}(\mathscr{E})$.

By "independent" we mean that there is a canonical isomorphism $\mathrm{K}_{*}(\mathcal{A}(\mathscr{E}, B)) \cong$ $\mathrm{K}_{*}\left(\mathscr{A}^{\prime}(\mathscr{G}, B)\right)$ whenever $\mathcal{A}^{\prime}(\mathscr{G})$ is another choice of an unconditional completion. 
We are not able to show this conjecture in full generality, yet, even for the case that the coefficients are trivial, i.e., $B=\ell_{0}(X)$. But in this section, we will give proofs for important cases:

Proposition A.2. If $\mathcal{G}$ is proper and if $\mathcal{A}(\mathcal{G})$ is either a regular or a strictly positive unconditional completion of $\mathcal{C}_{c}(\mathscr{G})$ and if $B$ is a $\mathscr{G}$-Banach algebra, then $\mathrm{K}_{*}(\mathcal{A}(\mathcal{G}, B))$ is independent of the choice of the (regular or strictly positive) unconditional completion $\mathcal{A}(\mathscr{E})$.

The notion of regularity was defined in Section 4.3. Strict positivity is a condition on the fibres $\mathcal{A}^{x}(\mathscr{G})$ of $\mathcal{A}(\mathscr{G})$ with respect to the left action by $\boldsymbol{C}_{0}(X)$ (i.e., the fibration with respect to the range map $r$ of $\mathscr{G}$ ) which is fairly elementary to check in concrete examples:

Definition A.3 (Strictly positive norm). Let $\mathcal{A}(\mathscr{G})$ be an unconditional completion of $\mathcal{C}_{c}(\mathscr{E})$ for a norm $\|\cdot\|_{\mathcal{A}}$. Then the norm $\|\cdot\|_{\mathcal{A}}$ and the completion $\mathcal{A}(\mathcal{E})$ are called strictly positive if for all compact $K \subseteq X$ there is a function $\chi \in \ell_{c}(\mathscr{E})_{\geq 0}$ such that $\inf _{x \in K}\|\chi\|_{\mathcal{A}^{x}}>0$.

Note that strict positivity is not symmetric in the sense that it treats the range and the source map differently. But in spite of this aesthetical drawback, this notion seems to be quite useful in concrete examples. As a start, if $\mathcal{E}=G$ is a group, then every unconditional norm on $\ell_{c}(G)$ is strictly positive. More generally:

Lemma A.4. If $\mathcal{E}=G \ltimes X$ where $G$ is a locally compact Hausdorff group acting (properly or not) on a locally compact Hausdorff space $X$, and if $\mathcal{A}^{\prime}(G)$ is an unconditional completion of $\mathcal{C}_{c}(G)$, then $\mathcal{A}(G \ltimes X):=\mathcal{A}^{\prime}\left(G, \bigodot_{0}(X)\right)$ is a strictly positive unconditional completion of $\ell_{c}(G \ltimes X)$.

Proof. Let $K \subseteq X$ be compact. Fix a function $\chi^{\prime} \neq 0$ in $\ell_{c}(G)_{\geq 0}$. Find a function $\chi \in \mathcal{C}_{c}(G \ltimes X)_{\geq 0}$ such that, for all $x \in K$ and $g \in G$, we have $\chi\left(g, g^{-1} x\right)=\chi^{\prime}(g)$ (if we fix the range map of the groupoid $G \ltimes X$ to send $(g, x)$ to $g x$ ). The fibre of $\mathcal{A}^{\prime}\left(G, \mathcal{C}_{0}(X)\right)$ over $x \in X$ can be identified with $\mathcal{A}^{\prime}(G)$, and this identification sends $\chi$ to $\chi^{\prime}$ for all $x \in K$. So, actually,

$$
\inf _{x \in K}\|\chi\|_{\mathcal{A}^{x}}=\left\|\chi^{\prime}\right\|_{\mathcal{A}^{\prime}}>0 .
$$

As an exercise, one can prove the following alternative characterisation of strict positivity:

Lemma A.5. Let $\mathcal{A}(\mathcal{G})$ be an unconditional completion of $\boldsymbol{C}_{c}(\mathcal{G})$ for a norm $\|\cdot\|_{\mathcal{A}}$. Then $\mathcal{A}(\mathcal{E})$ is strictly positive if and only if the following holds: for all $\chi \in \mathscr{C}_{c}(\mathcal{E})$ and all $\gamma \in \mathcal{E}$ with $\chi(\gamma) \neq 0$ there exists $U \subseteq X$ open with $r(\gamma) \in U$ such that $\inf _{x \in U}\|\chi\|_{\mathcal{A}^{x}}>0$. 
Also, note that $\mathrm{L}^{1}(\mathscr{E})$ is both, strictly positive and regular. But there are also regular unconditional completions which are not strictly positive and vice versa:

Examples A.6. (1) Let $X:=[0,1]$ and $\mathscr{E}:=X \times X$. Let the Haar system on $\mathscr{E}$ be induced from the Lebesgue measure on $X$. Define a norm on $\ell_{c}(\mathscr{E})$ by

$$
\|f\|_{\mathcal{A}}:=\sup _{y \in X} \int_{0}^{1}|f(x, y)| \mathrm{d} x .
$$

Then $\mathcal{A}(\mathscr{E})$ is an unconditional completion of $\mathcal{C}_{c}(\mathscr{E})$ which is not strictly positive. Note that it would be if we had taken the fibration with respect to the source map $s$ and not the range map $r$. Note, moreover, that $\mathcal{A}(\mathscr{E})=\mathrm{L}^{1}(\mathscr{E})^{*}$, so $\mathcal{A}(\mathscr{E})$ is regular.

(2) Let $\mathcal{E}$ and $X$ be as above, but this time consider the norm

$$
\|f\|_{\mathcal{B}}:=\||f|\|_{C^{*}} .
$$

Then $\mathcal{B}(\mathscr{E})$ is an unconditional completion of $\mathcal{C}_{c}(\mathscr{E})$ which is not strictly positive. As it dominates the $\mathrm{C}^{*}$-norm, it is regular.

(3) Let $\mathcal{G}$ be the group $\mathbb{Z}$ and let $\lambda \in \mathbb{R} \backslash\{0\}$. Define a norm on $\mathcal{C}_{c}(\mathbb{Z})$ by

$$
\|f\|_{\mathscr{D}}:=\sum_{k \in \mathbb{Z}} e^{\lambda k}|f(k)| .
$$

This is an unconditional norm on $\ell_{c}(\mathbb{Z})$. If it were regular, one could argue from (H2) and (H4) that $\left\|e_{l}\right\|_{\mathscr{D}}=1$ for all $l \in \mathbb{Z}$, but $\left\|e_{l}\right\|_{\mathscr{D}}=e^{\lambda l} \neq 1$. So $\mathscr{D}(\mathbb{Z})$ is not regular. It is, nevertheless, strictly positive.

Of course, one can combine these examples to obtain a completion which is neither regular nor strictly positive.

In the rest of the appendix, we will prove Proposition A.2. The strategy of the proof will be to check that the algebra $\Gamma_{c}\left(\mathcal{G}, r^{*} B\right)$ of continuous sections with compact support is dense and hereditary (see below) in the algebra $\mathcal{A}(\mathscr{G}, B)$ in question, which will give us the necessary information on the K-theory of $\mathcal{A}(\mathscr{E}, B)$.

In Section A.2, we will introduce some background knowledge on hereditary subalgebras and see how this concept can help us, and in Section A.3 and Section A.4 we then deal with the regular and the strictly positive case, respectively.

\section{A.2. Hereditary subalgebras and K-theory}

Definition A.7 (Hereditary subalgebra). Let $A$ be a subalgebra of a complex algebra $B$. Then $A$ is called hereditary in $B$ if $A B A \subseteq A$.

This concept was used in similar circumstances by Lafforgue in [Laf02], Section 1.7. We will use a slightly refined version of it: Actually, the algebra $\Gamma_{c}\left(\mathcal{G}, r^{*} B\right)$ does not necessarily have to be a subalgebra of its completion $\mathcal{A}(\mathscr{E}, B)$ because the 
canonical map $\iota$ from $\Gamma_{c}\left(\mathscr{E}, r^{*} B\right)$ to $\mathcal{A}(\mathcal{G}, B)$ does not have to be injective; the point is that the pointwise norm-function of an element of $\Gamma_{c}\left(\mathscr{G}, r^{*} B\right)$ does not have to be continuous, so it is conceivable that it has "norm" zero without vanishing. However, the kernel of $\iota$ can easily be seen to be nilpotent, which is good enough.

Lemma A.8. Let $\iota: A \rightarrow B$ be a homomorphism of complex algebras such that $\iota(A)$ is a hereditary subalgebra of $B$ and such that the kernel of $\iota$ is nilpotent. Then, for all $a \in A$, we have: if $1+\iota(a)$ is invertible in the unitalisation $\widetilde{B}$ of $B$, then $1+a$ is invertible in $\widetilde{A}$.

Proof. Let $a \in A$ such that $1+\iota(a)$ is invertible in $\widetilde{B}$. Let $1+b$ be the inverse of $1+\iota(a)$ in $\widetilde{B}$. Then, as in the proof of Lemma 1.7.9 of [Laf02] or Lemma 8.2.2 of [Par07], $b=-\iota(a)+\iota(a)^{2}+\iota(a) b \iota(a)$ belongs to $\iota(A)$. Find $a^{\prime} \in A$ such that $\iota\left(a^{\prime}\right)=b$. Then $\tilde{\iota}\left((1+a)\left(1+a^{\prime}\right)\right)=(1+\iota(a))\left(1+\iota\left(a^{\prime}\right)\right)=1=\tilde{\iota}\left(\left(1+a^{\prime}\right)(1+a)\right)$. This means that $(1+a)\left(1+a^{\prime}\right)=1+n$ for some $n$ in the kernel of $\iota$. But such an element is always invertible, so $1+a$ is right-invertible in $\widetilde{A}$. Similarly, $1+a$ is left-invertible in $\widetilde{A}$, so it is invertible.

Remark A.9. Note that Lemma A.8 is insensitive to amplification: If $n \in \mathbb{N}$ and $\iota: A \rightarrow B$ satisfies the hypotheses of the lemma, then also $\mathrm{M}_{n}(\iota): \mathrm{M}_{n}(A) \rightarrow \mathrm{M}_{n}(B)$ satisfies them. So the conclusion holds for $a \in \mathrm{M}_{n}(A)$ as well.

Lemma A.10. Let $\psi: B_{1} \rightarrow B_{2}$ be a continuous homomorphism of Banach algebras with dense image. Let $A$ be a dense subalgebra of $B_{1}$ such that $\psi(A)$ is a hereditary subalgebra of $B_{2}$ and such that the kernel of $\left.\psi\right|_{A}$ is nilpotent. Then $\psi$ is an isomorphism in $\mathrm{K}$-theory:

$$
\mathrm{K}_{*}\left(B_{1}\right) \cong \mathrm{K}_{*}\left(B_{2}\right) .
$$

Proof. Note that $\left.\psi\right|_{A}$ satisfies the conditions of Lemma A.8. This means that $\psi$ is relatively spectral in the sense of Nica with respect to the dense subalgebra $A$, see [Nic10], i.e., for every element $a$ in $A$ we have $\operatorname{sp}_{B_{1}}(\psi(a))=\operatorname{sp}_{B_{2}}(a)$. By the above remark, we can conclude that $\psi$ is even completely relatively spectral in the sense of Nica, so it follows from Theorem 1.2 of [Nic10] that $\psi$ is an isomorphism in K-theory.

If one does not want to invoke the machinery of [Nic10], one can also find a more direct proof along the lines of the proof of Lemma 1.7.10 of [Laf02].

Lemma A.11. Let $B$ be a complex algebra. For each submultiplicative semi-norm $p$ on $B$ let $B_{p}$ denote the completion of $B$ with respect to $p$ and let $\iota_{p}: B \rightarrow B_{p}$ denote the canonical "inclusion". Consider the class $C$ of all those $p$ such that $\iota_{p}(B)$ is hereditary in $B_{p}$ and such that $\iota_{p}$ has nilpotent kernel. Then $\mathrm{K}_{*}\left(B_{p}\right)$ does not depend on $p \in \boldsymbol{C}$. 
Proof. We first consider the case that we have $p$ and $q$ in $\mathcal{C}$ such that $q \leq p$. Let $\psi$ denote the canonical homomorphism from $B_{p}$ to $B_{q}$ and write $A$ for $\iota_{p}(B) \subseteq B_{p}$. Then $\psi: B_{p} \rightarrow B_{q}$ satisfies the hypotheses of Lemma A.10, so $\psi_{*}: \mathrm{K}_{*}\left(B_{p}\right) \rightarrow$ $\mathrm{K}_{*}\left(B_{q}\right)$ is an isomorphism.

If $p$ and $q$ are arbitrary in $\ell$, then we can consider $r:=\max \{p, q\}$, which is also in $\mathcal{C}$. Then we can apply the first part of the proof to $p$ and $r$ and $p$ and $r$ and $q$, respectively.

The link between the concept of a hereditary subalgebra and the proper groupoids and unconditional completions is given by the following lemma. In combination with Lemma A.11 it implies Proposition A.2.

Lemma A.12. Let $\mathscr{E}$ be proper and let $B$ be a $\mathscr{G}$-Banach algebra. Let $\mathcal{A}(\mathscr{E})$ be a regular or strictly positive unconditional completion and let $\iota$ denote the canonical map from $\Gamma_{c}:=\Gamma_{c}\left(\mathcal{E}, r^{*} B\right)$ into its completion $\mathcal{A}:=\mathcal{A}(\mathcal{E}, B)$. Then the threefold convolution product $\Gamma_{c} \times \Gamma_{c} \times \Gamma_{c} \rightarrow \Gamma_{c}$ can be (uniquely) extended to a trilinear separately continuous map $\Gamma_{c} \times \mathcal{A} \times \Gamma_{c} \rightarrow \Gamma_{c}$.

It follows that $\iota\left(\Gamma_{c}\right)$ is hereditary in $\mathcal{A}$ and that the kernel $N$ of $\iota$ satisfies $\Gamma_{c} N \Gamma_{c}=$ 0 ; in particular, $N$ is nilpotent.

Proof. To show that the threefold convolution product on $\Gamma_{c}$ extends to a trilinear separately continuous map $\Gamma_{c} \times \mathcal{A} \times \Gamma_{c} \rightarrow \Gamma_{c}$ will be shown in Section A.3 and Section A.4 in the regular and strictly positive case, respectively. Because of the continuity in the second component and the density of $\iota\left(\Gamma_{c}\right)$ in $\mathcal{A}$ this extension is unique.

From $\iota\left(\Gamma_{c}\right) \mathcal{A} \iota\left(\Gamma_{c}\right)=\iota\left(\Gamma_{c} \mathcal{A} \Gamma_{c}\right) \subseteq \iota\left(\Gamma_{c}\right)$ it follows that $\iota\left(\Gamma_{c}\right)$ is hereditary in $\mathcal{A}$. If $n \in \Gamma_{c}$ is in the kernel of $\iota$ and $f_{1}, f_{2} \in \Gamma_{c}$, then $f_{1} n f_{2}=f_{1} \iota(n) f_{2}=f_{1} 0 f_{2}=0$.

Remark A.13. The core of the argument why " $\Gamma_{c} * \mathcal{A} * \Gamma_{c} \subseteq \Gamma_{c}$ " holds does not involve the properness and, before we start with the actual proof, we want to explain it in the case that $\mathscr{E}=G$ is a (unimodular) group, that $B=\mathbb{C}$ and that $\mathcal{A}(G)=\mathrm{L}^{1}(G)$. The interesting part of what we have to show is that $\ell_{c}(G) * \mathrm{~L}^{1}(G) * \ell_{c}(G)$ is contained in $\mathcal{C}(G)$, the space of all continuous functions on $G$.

Firstly, consider $\mathcal{C}_{c}(G)$ as a subspace of $\mathrm{L}^{2}(G)$. Because $\mathrm{L}^{1}(G)$ acts on $\mathrm{L}^{2}(G)$ by convolution, canonically, we see that

$$
\ell_{c}(G) * \mathrm{~L}^{1}(G) * \ell_{c}(G) \subseteq \mathrm{L}^{2}(G) * \mathrm{~L}^{1}(G) * \mathrm{~L}^{2}(G) \subseteq \mathrm{L}^{2}(G) * \mathrm{~L}^{2}(G) \subseteq \ell_{0}(G) .
$$

The last inclusion can easily be shown using the Cauchy-Schwartz inequality. What we will show below for the regular case will be a generalisation of this idea.

Secondly and alternatively, one could proceed as follows: Show that already $\mathrm{L}^{1}(G) * \mathcal{\ell}_{c}(G) \subseteq \mathcal{\ell}(G)$; in this simple case, this can be achieved by regarding $\mathcal{C}_{c}(G)$ as a subset of $\mathcal{C}_{0}(G)$, on which $\mathrm{L}^{1}(G)$ acts canonically by convolution. Actually, it 
is true that $\mathcal{A}(G) * \mathcal{\ell}_{c}(G) \subseteq \mathcal{C}(G)$ for any unconditional completion $\mathcal{A}(G)$ if $G$ is a group. An argument for this fact has been indicated to me by Vincent Lafforgue, and a generalisation to strictly positive unconditional completions is what we show below in Section A.4.

A.3. Regular unconditional completions. Let $B$ be a $\mathcal{E}$-Banach algebra and let $\mathcal{A}(\mathscr{G})$ be a regular unconditional completion. Let $\mathcal{A}(\mathscr{G})$ act on the equivariant pair $\mathscr{H}(\mathcal{G})$ of locally convex monotone completions of $\mathcal{C}_{c}(\mathcal{E})$ satisfying $(\mathrm{H} 1)-(\mathrm{H} 4)$ as in Definition 4.12.

We now show that the threefold convolution product on $\Gamma_{c}:=\Gamma_{c}\left(\mathcal{H}, r^{*} B\right)$ can be extended to a trilinear separately continuous map from $\Gamma_{c} \times \mathcal{A}(\mathcal{E}, B) \times \Gamma_{c}$ to $\Gamma_{c}$ if $\mathcal{E}$ is proper.

Lemma A.14. Let $\mathcal{G}$ be proper and let $\mathcal{A}(\mathcal{G})$ be regular. Let $\iota$ be the canonical map from $\Gamma_{c}\left(\mathcal{G}, r^{*} B\right)$ to $\mathcal{A}(\mathcal{E}, B)$. Then $\iota\left(\Gamma_{c}\left(\mathscr{E}, r^{*} B\right)\right)$ is a hereditary subalgebra of $\mathcal{A}(\mathcal{E}, B)$ and the kernel $N$ of $\iota$ satisfies $\Gamma_{c}\left(\mathscr{G}, r^{*} B\right) N \Gamma_{c}\left(\mathscr{G}, r^{*} B\right)=0$; in particular, it is nilpotent with $N^{3}=0$.

Proof. Let $\mathcal{A}(\mathscr{G})$ act on the equivariant pair $\mathscr{H}(\mathscr{G})$ of locally convex monotone completions of $\mathcal{C}_{c}(\mathscr{G})$. Let $\beta^{<}, \beta^{>} \in \Gamma_{c}\left(\mathcal{G}, r^{*} B\right)$. Let $K_{r}:=r\left(\operatorname{supp} \beta^{<}\right)$and $K_{s}:=s\left(\operatorname{supp} \beta^{>}\right)$. The two sets $K_{r}$ and $K_{s}$ are compact subsets of $\mathscr{E}^{(0)}$. Because $\mathcal{E}$ is proper, the set $K:=\left\{\gamma \in \mathcal{E}: r(\gamma) \in K_{r}, s(\gamma) \in K_{s}\right\}$ is compact. For all $\beta \in \Gamma_{c}\left(\mathcal{E}, r^{*} B\right)$, we have $\operatorname{supp}\left(\beta^{<} * \beta * \beta^{>}\right) \subseteq K$. Because $\mathcal{A}(\mathcal{E})$ acts on $\mathscr{H}(\mathscr{E})$, we also have, by Lemma 4.11 and property $(\mathrm{H} 2)$ :

$$
\left\|\beta^{<} * \beta * \beta^{>}\right\|_{\infty} \leq\left\|\beta^{<}\right\|_{\mathscr{H}}<\|\beta\|_{\mathscr{A}}\left\|\beta^{>}\right\|_{\mathscr{H}}>
$$

It follows that $\left(\beta^{<} * \beta_{n} * \beta^{>}\right)_{n \in \mathbb{N}}$ is a Cauchy sequence in $\Gamma_{K}\left(\mathscr{E}, r^{*} B\right)$ whenever $\left(\beta_{n}\right)_{n \in \mathbb{N}}$ is a Cauchy sequence in $\Gamma_{c}\left(\mathscr{E}, r^{*} B\right)$ for the semi-norm $\|\cdot\|_{\mathcal{A}}$; in this case, $\left(\beta^{<} * \beta_{n} * \beta^{>}\right)_{n \in \mathbb{N}}$ converges to some element of $\Gamma_{K}\left(\mathcal{G}, r^{*} B\right)$, and hence $\iota\left(\beta^{<} * \beta_{n} * \beta^{>}\right)=\iota\left(\beta^{<}\right) \iota\left(\beta_{n}\right) \iota\left(\beta^{>}\right)$converges to some element in the image of $\iota$ if $n \rightarrow \infty$. Thus the image of $\iota$ is hereditary in $\mathcal{A}(\mathcal{G}, B)$.

Now let $\beta \in \Gamma_{c}\left(\mathcal{E}, r^{*} B\right)$ satisfy $\iota(\beta)=0 \in \mathcal{A}(\mathcal{G}, B)$. Let $\beta^{<}, \beta^{>}$be elements of $\Gamma_{c}\left(\mathscr{G}, r^{*} B\right)$. By $(\mathrm{H} 2),\left\|\beta^{<} * \beta * \beta^{>}\right\|_{\infty} \leq\left\|\beta^{<}\right\|_{\mathscr{H}}<\|\beta\|_{\mathcal{A}}\left\|\beta^{>}\right\|_{\mathscr{H}^{>}}=0$, so $\beta^{<} * \beta *$ $\beta^{>}=0$. This shows that the kernel $N$ of $\iota$ satisfies $\Gamma_{c}\left(\mathscr{G}, r^{*} B\right) N \Gamma_{c}\left(\mathscr{E}, r^{*} B\right)=0$.

Example A.15. Let $G$ be a locally compact Hausdorff group acting properly on some locally compact Hausdorff space $X$. Then $\mathrm{L}^{1}(G \ltimes X)$ and $\mathrm{L}^{1}\left(G, \bigodot_{0}(X)\right)$ are two regular unconditional completions of $\ell_{c}(G \ltimes X)$. Because $G \ltimes X$ is a proper groupoid, we have a canonical isomorphism

$$
\mathrm{K}_{*}\left(\mathrm{~L}^{1}\left(G, \bigodot_{0}(X)\right)\right) \cong \mathrm{K}_{*}\left(\mathrm{~L}^{1}(G \ltimes X)\right) .
$$


Because the unconditional norm given by $\mathrm{L}^{1}\left(G, \mathcal{C}_{0}(X)\right)$ dominates $\|\cdot\|_{1}$, the isomorphism in $\mathrm{K}$-theory is given by the canonical homomorphism from $\mathrm{L}^{1}\left(G, \mathcal{C}_{0}(X)\right)$ to $\mathrm{L}^{1}(G \ltimes X)$.

\section{A.4. Strictly positive unconditional completions}

Lemma A.16. For all $f \in \mathcal{C}(\mathscr{E})$ and $g \in \mathcal{C}_{c}(\mathscr{E})$ the function $f * g$ is well defined and continuous. The so defined product from $\mathcal{E}(\mathscr{E}) \times \mathcal{C}_{c}(\mathscr{E})$ to $\mathscr{C}(\mathscr{E})$ is separately continuous if we equip $\mathcal{C}_{c}(\mathscr{E})$ with the inductive limit topology and $\mathscr{C}(\mathscr{E})$ with the topology of uniform convergence on compact sets. The same holds for the product with the factors reversed.

Let $\mathcal{A}(\mathscr{E})$ be an unconditional completion of $\mathcal{C}_{c}(\mathscr{E})$ for an unconditional strictly positive norm $\|\cdot\|_{\mathcal{A}}$, see Definition A.3.

Lemma A.17. The convolution on $\mathcal{C}_{c}(\mathscr{G})$ extends to a well-defined product from $\mathcal{A}(\mathscr{E}) \times \mathcal{C}_{c}(\mathscr{E})$ to $\mathscr{C}(\mathscr{E})$ which is separately continuous.

Proof. Let $K$ be a compact subset of $\mathscr{G}$. We show that the convolution from $\mathcal{C}_{c}(\mathscr{E}) \times$ $\mathcal{C}_{K}(\mathscr{E})$ to $\mathscr{C}(\mathscr{E})$ extends continuously to a map on $\mathcal{A}(\mathscr{E}) \times \mathcal{C}_{K}(\mathscr{E})$. This would show separate continuity for the extension of the convolution to a map on $\mathcal{A}(\mathscr{E}) \times \mathscr{C}_{c}(\mathscr{E})$.

So let $L$ be a compact subset of $\mathscr{G}$. We have to show that the map $(f, g) \mapsto$ $\left.(f * g)\right|_{L}$ from $\mathscr{C}_{c}(\mathscr{G}) \times \mathcal{C}_{K}(\mathscr{E})$ to $\mathscr{C}(L)$ is bounded. Because the norm on $\mathcal{A}(\mathscr{E})$ is strictly positive, we can find a function $\chi \in \mathscr{C}_{c}(\mathscr{\mathscr { S }})_{\geq 0}$ such that $\inf _{x \in r(L)}\|\chi\|_{\mathcal{A}_{x}}>0$ and $0 \leq \chi \leq 1$. Define $V:=\operatorname{supp} \chi$.

Note that the set $K L^{-1} V$ is a compact subset of $\mathcal{G}$, so we can find a function $\psi \in \mathscr{C}_{c}(\mathscr{E})$ such that $0 \leq \psi \leq 1$ and $\psi \equiv 1$ on $K L^{-1} V$. We define

$$
C_{K, L}:=\frac{\|\psi\|_{\mathcal{A}}}{\inf _{x \in r(L)}\|\chi\|_{\mathcal{A}_{x}}} .
$$

Let $f \in \mathcal{C}_{c}(\mathscr{E})$ and $g \in \mathcal{C}_{K}(\mathscr{E})$ and $x \in \mathscr{E}^{(0)}$.

We have

$$
\left|\psi\left(s^{-1} v\right)\right|\|g\|_{\infty} \geq\left|g\left(s^{-1} l\right)\right| .
$$

for all $v \in V$, for all $l \in L$ and all $s \in \mathcal{E}$ such that $r(s)=r(v)=r(l)=x$ : If $s^{-1} l$ is in supp $g \subseteq K$, then this means that $s^{-1} v=s^{-1} l l^{-1} v$ is in $K L^{-1} V$, so $\psi\left(s^{-1} v\right)=1$ and the above inequality holds in this case. If $s^{-1} l$ is not in supp $g$, then the inequality holds trivially.

Now for all $l \in L$ and $v \in V$ such that $r(v)=r(l)=x$ we have

$$
\begin{aligned}
\|g\|_{\infty}|f| *|\psi|(v) & =\int_{s \in \mathscr{G}^{x}}|f|(s)|\psi|\left(s^{-1} v\right)\|g\|_{\infty} \mathrm{d} s \\
& \geq \int_{s \in \mathcal{E}^{x}}|f|(s)|g|\left(s^{-1} l\right) \mathrm{d} s=|f| *|g|(l) \geq[|f| *|g|(l)] \chi(v) .
\end{aligned}
$$


Because supp $\chi=V$, the same inequality holds for all $v \in \mathcal{E}^{x}$. Because the norm $\|\cdot\|_{\mathcal{A}}$ is unconditional, we have \|\|$g\left\|_{\infty}|f| *|\psi|{\| \mathcal{A}_{x}} \geq[|f| *|g|(l)]\right\| \chi \|_{\mathcal{A}_{x}}$. This implies that

$$
\|g\|_{\infty}\|f\|_{\mathcal{A}_{x}}\|\psi\|_{\mathcal{A}} \geq[|f| *|g|(l)]\|\chi\|_{\mathcal{A}_{x}}
$$

for all $l \in L$.

$$
|(f * g)(l)| \leq[|f| *|g|(l)] \leq \frac{\|\psi\|_{\mathcal{A}}}{\|\chi\|_{\mathcal{A}_{x}}}\|g\|_{\infty}\|f\|_{\mathcal{A}_{x}} \leq C_{K, L}\|g\|_{\infty}\|f\|_{\mathcal{A}} .
$$

This is true for all $l \in L$, so

$$
\|f * g\|_{L} \leq C_{K, L}\|g\|_{\infty}\|f\|_{\mathcal{A}} .
$$

This is the continuity property that we wanted to prove.

Let $\mathcal{G}$ be a proper locally compact Hausdorff groupoid. Let $B$ be a $\mathcal{G}$-Banach algebra. Define $\Gamma_{c}:=\Gamma_{c}\left(\mathcal{E}, r^{*} B\right)$. Let $\mathcal{A}(\mathcal{G})$ be a strictly positive unconditional completion of $\boldsymbol{C}_{c}(\mathcal{G})$.

Corollary A.18. Let $f_{1}, f_{3} \in \Gamma_{c}:=\Gamma_{c}\left(\mathcal{E}, r^{*} B\right)$. Then the map $f_{2} \mapsto f_{1} * f_{2} * f_{3}$ from $\Gamma_{c}\left(\mathscr{E}, r^{*} B\right)$ to $\Gamma_{c}\left(\mathscr{E}, r^{*} B\right)$ extends to a continuous linear map from $\mathcal{A}(\mathscr{E}, B)$ to the Banach space $\Gamma_{K}\left(\mathcal{E}, r^{*} B\right) \subseteq \Gamma_{c}$, where $K \subseteq \mathcal{E}$ is the compact set $\{\gamma \in \mathcal{E}$ : $r(\gamma) \in s\left(\operatorname{supp} f_{1}\right)$ and $\left.s(\gamma) \in r\left(\operatorname{supp} f_{3}\right)\right\}$.

In particular, the image of $\Gamma_{c}$ in its completion $\mathcal{A}(\mathcal{E}, B)$ is a hereditary subalgebra. Moreover, the kernel $N$ of the embedding of $\Gamma_{c}$ into $\mathcal{A}(\mathcal{E}, B)$ satisfies $\Gamma_{c} N \Gamma_{c}=0$, so in particular we have $N^{3}=0$.

Proof. Because $\mathcal{E}$ is proper, the set $K$ is compact.

It is clear that $\operatorname{supp}\left(f_{1} * f_{2} * f_{3}\right) \subseteq K$ for all $f_{2} \in \Gamma_{c}$. Moreover, we have shown above that

$$
\left\|f_{1} * f_{2} * f_{3}\right\|_{K} \leq\left\|\left|f_{1}\right| *\left|f_{2}\right| *\left|f_{3}\right|\right\|_{K} \leq C\left\|f_{2}\right\|_{\mathcal{A}},
$$

where the constant $C$ does only depend on $f_{1}$ and $f_{3}$. This shows the first part of the corollary.

The same inequality, for varying $f_{1}$ and $f_{3}$ (and hence for varying $C$ and $K$ ) shows the second part of the corollary.

\section{References}

[Bla98] B. Blackadar, K-theory for operator algebras. Math. Sci. Res. Inst. Publ. 5, 2nd ed., Cambridge University Press, Cambridge 1998. Zbl 0913.46054 MR 1656031

[Blan96] É. Blanchard, Déformations de $C^{*}$-algèbres de Hopf. Bull. Soc. Math. France 124 (1996), 141-215. Zbl 0851.46040 MR 1395009 
[Bos90] J.-B. Bost, Principe d'Oka, $K$-théorie et systèmes dynamiques non commutatifs. Invent. Math. 101 (1990), 261-333. Zbl 0719.46038 MR 1062964

[Gie82] G. Gierz, Bundles of topological vector spaces and their duality. Lecture Notes in Math. 955, Springer-Verlag, Berlin 1982. Zbl 0488.46060 MR 674650

[KS03] G. Kasparov and G. Skandalis, Groups acting properly on "bolic" spaces and the Novikov conjecture. Ann. of Math. (2) 158 (2003), 165-206. Zbl 1029.19003 MR 1998480

[Laf02] V. Lafforgue, $K$-théorie bivariante pour les algèbres de Banach et conjecture de Baum-Connes. Invent. Math. 149 (2002), 1-95. Zbl 1084.19003 MR 1914617

[Laf06] V. Lafforgue, $K$-théorie bivariante pour les algèbres de Banach, groupoïdes et conjecture de Baum-Connes. J. Inst. Math. Jussieu 6 (2007), 415-451. Zbl 1135.19002 MR 2329760

[Nic10] B. Nica, Spectral morphisms, K-theory, and stable ranks. In Perspectives on noncommutative geometry, Fields Inst. Commun. 61, Amer. Math. Soc., Providence, RI, 2011, 103-116. Zbl 1243.46059 MR 2838683

[Par07] W. Paravicini, KK-theory for Banach algebras and proper groupoids. $\mathrm{PhD}$ thesis, Universität Münster, Münster 2007. Zbl 1131.19002 http://miami.uni-muenster.de/servlets/DocumentServlet?id=3482

[Par08] W. Paravicini, A note on Banach $\bigodot_{0}(X)$-modules. Münster J. Math. 1 (2008), 267-278.

Zbl 1167.46047 MR 2502501 http://wwwmath.uni-muenster.de/mjm/vol1.html

[Par09a] W. Paravicini, Induction for Banach algebras, groupoids and KK ${ }^{\text {ban }}$. J. K-Theory 4 (2009), 405-468. Zbl 1189.19004 MR 2570950

[Par09b] W. Paravicini, Morita equivalences and KK-theory for Banach algebras. J. Inst. Math. Jussieu 8 (2009), 565-593. Zbl 1177.19003 MR 2516306

[Par13a] W. Paravicini, The spectral radius in $\ell_{0}(X)$-Banach algebras. J. Noncommut. Geom. 7 (2013), 135-147.

[Par13b] W. Paravicini, The Bost conjecture and proper Banach algebras. J. Noncommut. Geom. 7 (2013), 191-202.

[Ren80] J. Renault, A groupoid approach to $C^{*}$-algebras. Lecture Notes in Math. 793, Springer-Verlag, Berlin 1980. Zbl 0433.46049 MR 584266

[Tu99] J. L. Tu, La conjecture de Novikov pour les feuilletages hyperboliques. $K$-Theory 16 (1999), 129-184. Zbl 0932.19005 MR 1671260

[Tu04] J.-L. Tu, Non-Hausdorff groupoids, proper actions and $K$-theory. Doc. Math. 9 (2004), 565-597. Zbl 1058.22005 MR 2117427

Received December 2, 2010

W. Paravicini, Mathematisches Institut, Universität Münster, Einsteinstr. 62, 48149 Münster, Germany

E-mail: W.Paravicini@uni-muenster.de 